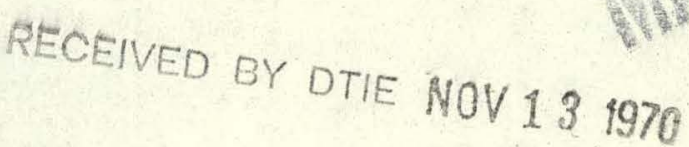

\section{ESTIMATION OF THE MEAN OF SKEWED DISTRIBUTIONS \\ USING SYSTEMATIC STATISTICS}

$\mathrm{Ph}$. D. Thesis Submitted to Iowa State University, August 1970

Donald Hughes McElhone and Wayne Fuller

\author{
Ames Laboratory, USAEC \\ Iowa State University
}

Ames, Iowa 50010

PREPARED FOR THE U.S. ATOMIC ENERGY COMMISSION UNDER CONTRACT NO. W-7405-eng-82

Date Transmitted: October 1970

This report LEAL NOTICE

sponsored by the prepared as an account of work

the United States United States Government. Neither

the United States nor the United States Atomt. Neither

Commission, nor any of their emples Atomic Energy

their contractors, subcontor their employees, nor any of

makes any warranty,

legal liability or responses or implied, or assumes any

pleteness or usefulness of any for the accuracy, com-

product or process disclo any information, apparatus,

would not infringe privately owned reprents that its use 


\section{DISCLAIMER}

This report was prepared as an account of work sponsored by an agency of the United States Government. Neither the United States Government nor any agency Thereof, nor any of their employees, makes any warranty, express or implied, or assumes any legal liability or responsibility for the accuracy, completeness, or usefulness of any information, apparatus, product, or process disclosed, or represents that its use would not infringe privately owned rights. Reference herein to any specific commercial product, process, or service by trade name, trademark, manufacturer, or otherwise does not necessarily constitute or imply its endorsement, recommendation, or favoring by the United States Government or any agency thereof. The views and opinions of authors expressed herein do not necessarily state or reflect those of the United States Government or any agency thereof. 


\section{DISCLAIMER}

Portions of this document may be illegible in electronic image products. Images are produced from the best available original document. 
IS -2205

LEGAL NOTICE

This report was prepared as an account of work sponsored by the United States Government. Neither the United States nor the United States Atomic Energy Commission, nor any of their employees, nor any of their contractors, subcontractors, or their employees, makes any warranty, express or implied, or assumes any legal liability or responsibility for the accuracy, completeness or usefulness of any information, apparatus, product or process disclosed, or represents that its use would not infringe privately owned rights.

Printed in the United States of America

Available from

Clearinghouse for Federal Scientific and Technical Information National Bureau of Standards, U.S. Department of Commerce Springfield, Virginià 22151

Price: Printed Copy $\$ 3.00 ;$ Microfiche $\$ 0.65$ 
ESTIMATION OF THE MEAN OF SKEWED DISTRIBOTIONS

USING SYSTEMATIC STATISTICS

Donald Hughes McElhone and Wayne Fuller

ABSTRACT

This paper investigates tho use of simple Iinoar combinations of order statistics to ostimate the mean of the Weibull distribution. Formulas for calculating the expected values, variances, and covariances of the order statistics of Welbull random variables with integer shape parameter are developed. The asymptotic values of the expectations and covariances are derived for the largest order statistics. These results aro used to study estimatore of the mean of the form $\hat{w}=\left(\sum_{1=1}^{n-r} \mathrm{~J}_{(1)}+r \mathrm{y}_{(n-r)}\right) / 0$ where the $\mathrm{J}_{(1)}$ are the ordered observations of a sample of size $n$ and $D$ is a suitably chosen denominator. This typo of estimator replaces each of the $r$ largest observations with the $(r+1)$-st largest obserration. In partfcular the WInsorized moan is tho ostimate $\$$ with $D=n$. Two othor forms of $D$ are considerod; $D=n-r$ and $D=n-r+1$. For the exponential $D=n-r$ gives an unbiased estimator and $D=n-r+1$ gives the minimum mean square error estimator. 
These three estimates are compared using mean square error as the criterion. The as ymptotic results are used to show that the mean square error of the once censored estimate is less than the mean square error of $\overline{\mathrm{J}}$ for Weibull distributions with shape parameter greater than one. The maximum likelihood estimate for the mean is developed and compared with $\overline{\mathbf{J}}$.

Tests of the null hypothesis that the shape parameter of the Weibull is one are investigated. A test suggested by Fuller, a test based on the log-Welbull estimator and the likelihood ratio test are compared. Fuller's test has the advantage of having a known distribution, but the power of Fuller's test is somewhat less than that of the log-Weibull test. The likelihood ratio test was more powerful than either, but the likelihood test studied is a simple versus simple test while the others are simple versus composite tests.

A technique is also presented which allows the calculation of expected values and expected crossproducts of the order statistics of symetric distributions using the expected values and crossproducts of the order statistics of the related half distribution. 
TABLE OF CONTENTS

ABSTRACT

CHAPTER I. INTRODUCTION AND IITERATURE REVIEW

Page

iii

1

CHAPTER II. WEIBULL ORDER STATISTICS 22

CHAPTER III. ASMMPOTIC MOMITS POR THE MEIBULC 40

CHAPTER IV. THE EXPON ETIAI DISTRIBUTIOR 53

CHAPTER V. ESTIMATION AND TESTING IN THE WEIBULL. DISTRIBUTION

62

CEAPTER VI. MOMENTS OF SMAIETRIC DISTRIBUTIONS FROM TEFIR HAIF DISTRIBUTIONS

CRAPTER VII. SUMMARI

BIBLIOGRAPHI

APPENDIX 
CHAPTER I. INTRODUCTION AND LITERATURE REVIEW

In this thesis we will study testing and estimation for the mean of a class of skewed distributions. The estimators used w1ll be systematic statistics, defined to be linear combinations of the order statistics of the sample. Let $x_{1}$ be the observations in a sample of size $n$. If they are put in increasing order, we shall denote the 1-th largest order statistic by $x_{(1)}$. Then $x_{(1)}$ is greater than or equal to $x(1-1)$. A review of the literature is provided later in th1s chapter. In Chapter. II we w1ll develop a procedure for numerical calculation of expected values and covariances (Including variances) of Welbull order statistics. In Chapter III, the asymptotic case is considered and asymptotic expected values and covarlances for the highest order statistics are developed. Chapter IV considers the special case of the exponential distribution. Chapter $V$ investigates comparative efficiency of several estimates of parameters of the Welbull. In addition we have studied three tests of departure from the exponential where the alternative is the Welbull distribution. In Chapter VI we will develope a combinatorial form which generates the moments of a symmetric distribution from those of the related half distribution. In particular, tables of the expected values and expected cross products of the double Welbull are given. 


\section{A. L1terature Review}

For a discussion of the properties of order statistics, see WIIks (48, 49). The book edited by Sarhan and Greenberg (37) covers the general topic of order statistics by presenting many of the important papers on the subject Including general theory and several articles on particular distributions.

The following result in Renyl (32) plays an important role in this thesis. If $X$ is an exponential random variable with parameter $b$, that $13, f(x)=b^{-1} e^{-x / b}$, let $x(1)$ be the ordered random varlables for a sample of $\mathbf{n}$ independent observations. Renyl presents a heuristic proof that the random varlables

$$
z_{k}=(n-k+1)\left(x_{(k)}-x_{(k-1)}\right) k=1,2, \ldots, n \quad(1.1)
$$

where $X(0) \equiv 0$, are 1ndependent, 1dent1cally distributed as exponential with parameter $b$. We present an elementary nonheuristic proof of this important result.

It is known that the joint density of the order statistics of a sample of size $n$ is

$$
\left.f\left(x_{(1)}, x_{(2)}, \ldots, x_{(n)}\right)=n ! f\left(x_{(1)}\right) f\left(x_{(2)}\right) \ldots f_{(n)}\right) \text {. }
$$

For the exponential density this becomes

$$
f\left(x(1), x_{(2)}, \ldots, x_{(n)}\right)=n ! b^{-n} \exp \left(-b^{-1} \sum_{i=1}^{n} x_{(1)}\right)
$$


Consider the transformation of Equation 1.1.

$$
\begin{aligned}
& z_{1}=n x_{(1)} \\
& z_{2}=(n-1)\left(x_{(2)}-x_{(1)}\right) \\
& \dot{z}_{k}=(n-k+1)\left(x_{(k)}-x_{(k-1)}\right) \\
& \dot{z}_{n}=\left(x_{(n)}-x_{(n-1)}\right)
\end{aligned}
$$

The inverse transformation is

$$
\begin{aligned}
& x_{(1)}=z_{1} / n \\
& x_{(2)}=z_{1} / n+z_{2} /(n-1) \\
& \dot{x}_{(k)}=z_{1} / n+z_{2} /(n-1)+\ldots+z_{k} /(n-k+1) \\
& \dot{x}_{(n)}=z_{1} / n+z_{2} /(n-1)+\ldots+z_{n} .
\end{aligned}
$$

The Jacobian of the transformation is given by

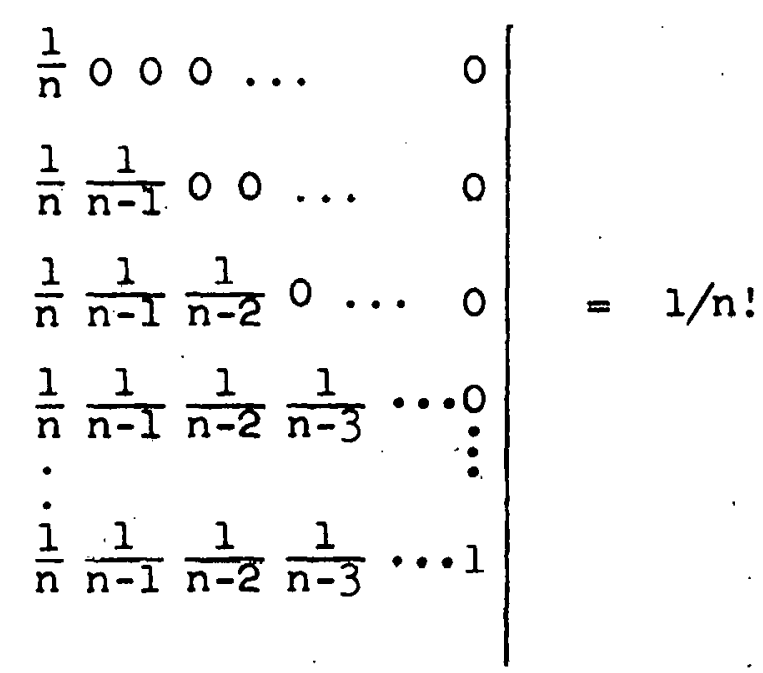


Therefore the foint density of the $z_{k}$ is given by

$$
\begin{aligned}
f\left(z_{1},\right. & \left.z_{2}, \ldots, z_{n}\right)=f\left(\frac{z_{1}}{n}\right) f\left(\frac{z_{1}}{n}+\frac{z_{2}}{n-1}\right) \ldots f\left(\sum_{1=1}^{n} z_{1} /(n-1+1)\right) \\
& =b^{-n} \exp \left(-b^{-1} z_{1} / n\right) \ldots \exp \left(-b^{-1} \sum_{1=1}^{n} z_{1} /(n-1)\right) \\
& =b^{-n} \exp \left(-b^{-1}\left(z_{1}+z_{2}+\ldots+z_{n}\right)\right) \\
& =\prod_{1=1}^{n}\left(b^{-1} \exp \left(-b^{-1} z_{1}\right)\right) .
\end{aligned}
$$

Therefore the random variables $Z_{k}$ are independent and Identically distributed as exponential with parameter $b$. Furthermore since $x_{(j)}=\sum_{1=1}^{j} z_{1} /(n-1+1), x_{(j)}$ is independent of $z_{k}$ if $j<k$.

Walodd1 Welbul1, a Swedish engineer, in 1939 (46), developed a distribution for the study of breaking strengths. This distribution has come to be known by his name. There are many equivalent forms of the distribution, but we shall write it as

$$
F(t ; a, c)=1-\exp \left(-(t / c)^{1 / a}\right) \quad t>0, a>0, c>0 \text {. }
$$

Normally the shape parameter of the Weibull is the reciprocal of the manner in which we will use it. The density function is

$$
\begin{aligned}
f(t ; a, c) & =\frac{t^{1 / a-1}}{a c^{1 / a}} \exp \left(-(t / c)^{1 / a}\right) t>0 \\
& =0 \text { otherwise. }
\end{aligned}
$$

The parameter $c$ is called the scale parameter or characteristic 1 ife and is the $100\left(1-e^{-1}\right)$ percent point of the 
distribution. We call the parameter a the shape parameter. As a decreases, the mode of the density approaches $c$ and, in the limit the density becomes degenerate at $c$.

An often used function when studying the Welbuil is the reliability function $R(t)=1-F(t)$. Johns and Lleberman (25) present a technique for contructing confidence intervals for $R(t)$ based on a transformed distribution. Consider a reparameterization of Equation 1.7 by letting $c=e^{v}$. The distribution function becomes

$$
F_{T}(t ; a, v)=1-\exp (-\exp ((\log t-v) / a))
$$

Let $t_{0}$ be the time at which the reliability is to be estimated and let $T^{\prime}=T / t_{O}$. Hence

$$
\begin{aligned}
F_{T^{\prime}}(t ; a, v) & =1-\exp \left(-\exp \left(\left(\log t-\left(v-\log t_{0}\right) / a\right)\right)\right) \\
& =F_{T}\left(t ; v-\log t_{0}, a\right) .
\end{aligned}
$$

so that $T^{\prime}$ is a Welbull varlate. Let $m=v-10 g t_{0}$, then $F_{T^{\prime}}(t ; a, m)=F_{T}(t ; a, m)$ and $R\left(t_{O}\right)=\exp (-\exp (-m / a))$. Thus, finding a lower confidence bound for $\mathrm{m} / \mathrm{a}$ is equivalent to finding a lower confidence bound for $R\left(t_{0}\right)$. Let $Y=\log T^{\prime}$, then

$$
P(Y<t)-P\left(T^{\prime}<e^{t}\right)=1-\exp (-\exp ((t-m) / a)) .
$$

Let $Y_{(1)}=\log T_{(1)}-\log t_{0}$ be the order statistics of the : sample and consider estimates for $m$ and a 


$$
\begin{aligned}
& Z_{a}=\stackrel{r}{\Sigma} \cdot A_{1} Y(1) \\
& Z_{b}=\stackrel{r}{\Sigma} B_{1} Y(j)
\end{aligned}
$$

where $\stackrel{r}{\Sigma} A_{1}=1, \stackrel{r}{\Sigma} B_{1}=0$, and $r$ is a constant percent amount of censoring. Johns and Lieberman (25) give tables of the $A_{1}$ and $B_{1}$ such that the quantities

$$
\begin{aligned}
& v_{a}=\frac{1}{a}\left(z_{a}-m\right) \\
& v_{b}=\frac{1}{a} z_{b} .
\end{aligned}
$$

have joint distribution which is parameter free and asymptoticaliy normal. Define $I(t)$ so that for fixed $Y, 0<\gamma<1$

$$
P\left(L(t)<t V_{b}-V_{a}\right)=\gamma
$$

for all t.

$$
\text { Suppose } P\left(V_{b}>0\right)=1 \text {, then } L(t) \text { is a monotone increasing }
$$
function of $t$ and $P\left(L\left(Z_{a} / Z_{b}\right)<m / a\right)=\gamma$. Therefore $L\left(Z_{a} / Z_{b}\right)$ Is a lower confldence bound for $\mathrm{m} / \mathrm{a}$ having confidence coefflclent $y$. Hence for the welbull, the corresponding lower confidence bound for $R\left(t_{0}\right)$ is

$$
L^{*}\left(Z_{a} / Z_{b}\right)=\exp \left(-\exp \left(-L\left(Z_{a} / Z_{b}\right)\right)\right)
$$

The procedure, then, is to compute $\mathrm{Z}_{a} / \mathrm{Z}_{\mathrm{b}}$ from the sample data and given the confldence coefflcient $Y$, use the tabular values of $L^{*}\left(Z_{a} / Z_{b}\right)$ to find the lower confidence bound. A point estimate for the reliability is given by $R^{*}\left(t_{0}\right)=$ 
$\exp \left(-\exp \left(-z_{a} / z_{b}\right)\right)$

Mann (30) has shown that if the first $m$ of $n$ sample values are observable then, for the Welbull, the set of the first $m$ order statistics is minimaliy sufficient. Furthermore, for $m$ greater than two, no joint complete sufficient statistics exist and, therefore, no unique minimum variance unblased estimates for the parameters exist under the Lehman-Scheffe theorem. Mann gives estimators based on just the first two order statistics.

If we consider the standardized Weibull by letting $z=$ $t / c$, we have

$$
\begin{aligned}
g(z ; a) & =\frac{z^{\frac{1}{a}}-1}{a} \exp \left(-z^{1 / a}\right), z>0, \\
& =0 \quad \text { otherwise. }
\end{aligned}
$$

The distribution function is given by

$$
a(z ; a)=1-\exp \left(-z^{1 / a}\right) .
$$

Splitstone (40) shows that for the above density function, the curve has a maximum at $(1-a)^{a}$ and inflection points at

$$
z_{\text {inf } 1}=\frac{3(1-a) \pm \sqrt{(5-a)(1-a)^{2}}}{2}
$$

and discusses four cases for the parameter a. We will be primarily interested in the case where the shape parameter a is greater than one. In this case the density function is convex over its entire domain. The second case is for a equal to one which is the usual exponential density. Case three 
is for a in the half closed interval $[1 / 2,1)$. In th. case the density has a mode and is concave below the mo. and convex for $z$ greater than the mode. Finally, for a less than one-half the density has a mode and an inflect:point on either side of the mode.

The median of the density is at $t=c(\log 2)^{2}$, where log will denote the natural logarithm throughout this thesis.

The moments of the distribution are found in the usual manner.

$$
E\left(t^{m}\right)=\int_{0}^{\infty}\left(t^{m^{\frac{1}{2}-1}} \exp \left(-(t / c)^{1 / a}\right) / a c^{1 / a}\right) d t .
$$

Let $z=(t / c)^{1 / a}$ and the integral becomes

$$
\int_{0}^{\infty} c^{m} z^{a m} \exp (-z) d z
$$

so that

$$
E\left(t^{m}\right)=c^{m} \Gamma(a m+1)
$$

$\Gamma$ is the gamma function. In particular, for $q$ an integer $\Gamma(q+1)=q !$.

There is a great volume of literature avallable on the Welbuli distribution. We begin the discussion of estimation by considering the maximum likelihood estimators (MLE). Let $b=c^{1 / a}$ then we write the density as

$$
\left.f(t ; a, b)=\frac{t^{\frac{1}{a}}-1}{a b} \exp \left(-t^{1 / a} / b\right)\right) \text {. }
$$


The log-likelihood function is then

$$
\log L=-n \log a-n \log b+\Sigma\left(\frac{1}{a}-1\right) \log t_{1}-\frac{1}{b} \Sigma t_{1}{ }^{1 / a}
$$

Taking the partial derlvatives with respect to $a$ and $b$ and setting them equal to zero we have the MLE of $a$ and $b$ as the solution of

$$
\begin{aligned}
& \hat{b}=\frac{1}{n} \Sigma t^{1 / \hat{a}} \\
& -\hat{a}+\frac{1}{n b} \Sigma t_{1}^{1 / \hat{a}} \log t_{1}=\frac{1}{n} \Sigma \log t_{1} .
\end{aligned}
$$

The estimator for $c$ is then

$$
\hat{c}=\left(\frac{1}{n} \Sigma t_{1}^{1 / \hat{a}}\right)^{\hat{a}} \text {. }
$$

It has been shown that the maximum likelihood estimators are consistent, asymptotically normal, and asymptotically efficient. Methods for solution are given by Harter and Moore (22) for both the censored and noncensored cases using initial estimates as starting points. Halperin (19) discusses a general procedure for a constant percentage of censoring. leading to estimates with the same properties as the MLE. Menon (31) uses the transformation $w=(t / c)^{1 / a}$ to get to the standard exponential variate and arrives at the estimate for a

$$
\hat{a}=\left(\frac{6}{\pi^{2}}\left(\Sigma \log t_{1}^{2}-\left(\Sigma \log t_{1}\right)^{2} / n\right) /(n-1)\right)^{1 / 2}
$$


which has asymptotic efficiency of about 55 percent. For the case of a known scale parameter he gives an unblased estimator of a which has symptotic efficiency of approximately 84 percent. Menon's procedure does not use order statistics and is not applicable to the case of censored samples.

In the area of least square estimation applied to order statistics, Lloyd (27) studied distributions which are dependent on location and scale parameters only. If the expectations and covarlances (and varlances) of the order statistics are known, then generalized least squares performed on the order statistics give minimum variance, unblased Iinear estimates. Let $y_{1}, 1=1,2, \ldots, n$, be a sample of $n$ independent observations and $y_{(1)}$ be the ordered observations. Let $u_{(1)}=\left(y_{(1)}-\mu\right) / 0$ be a standardized observation with expectation $E\left(u_{(1)}\right)=a_{1}$ and $\operatorname{cov}(u(1) u(j))=w_{1 j}$. The $\alpha_{1}$ and $w_{1 j}$ are dependent on the parent distribution, but not on $\mu$ or $\sigma$. Then In lerms of the original observations $E\left(y_{(1)}\right)=u+\sigma r_{1}$ and $\operatorname{cov}\left(y_{(1)} y_{(j)}\right)=\sigma^{2} w_{1 j}$. If we write these equations in matrix notation by letting $Y$ be the vector of ordered observations, then

$$
E(Y)=\omega Z+\sigma \alpha=p \theta
$$

where $\alpha$ is the vector of $a_{1}$. $\frac{1}{\sim}$ is a vector of ones, $p=$ $\left(\frac{1}{\sim}, \alpha\right)$, and hence $\theta^{\prime}=(\mu, \sigma)$. Furthermore 


$$
V(Y)=\sigma^{2} w
$$

where $w$ is an nxn positive definite, symmetric matrix with typical element $w_{1 j}$. The estimator is given by

$$
\hat{\theta}=\left(p^{\prime} w^{-1} p\right)^{-1} p^{\prime} w^{-1} Y
$$

and

$$
V(\hat{\theta})=\sigma^{2}\left(p^{\prime} w^{-1} p\right)^{-1}
$$

Lloyd further shows that the varlance of the location parameter, $V(\hat{\mu}) \leq \sigma^{2} / n$. Sarhan (36) uses Lloyd's procedure to derive the particular estimates for the rectangular, triangular, double, and single exponential distributions.

Blom (2) considers a class of estimates which he calls "nearly best estimates". These are estimates with nearly minimum variance. Let $Z$ be a random variable with a known continuous distribution, $F((z-a) / b)$, and let $Z(1)$ be the ordered random variables.from a sample of $n$. soppose the expectations and covariances of the ordered rundom variables are not known. Blom suggests making a transformation such that the transformed variables are approximately distributed as functions of uniform random variables. The expected values and covarlances of the order statistics of the transformed variables as well known. He then performs least squares on the transformed variables and uses the results to give estimates for the parameters of the original distribution. 
We concern ourselves now with censored estimation. In this thesis we shall say that an estimate 1s. censored if the extreme observations are not used in estimation whether they are avallable or not. However, the slze of the whole sample 1s always known.

David and Johnson (4) make an expansion to approximate the moments of the ordered observations. Unfortunately, the expansion fails for the highest and the lowest order statistics. Their procedure is satisfactory for percentile censoring although the formulas are rather complex. A best linear systematic statist1c is derived by performing least squares on the order statistics of the sample. We denote the best linear systemat1c stat1st1c by BLSS. Sarhan and Greenberg (38) use the BLSS to study the normal distribution for sample sizes up to ten which are singly and double censored. They give tables of the expectations and covariances for the estimator. For the noncensored case the estimator $\Sigma x_{(1)} / n$, the usual mean, is the BLSS. The estimators are of the form

$$
p=\sum_{1=r_{1}+1}^{n-r_{2}} a_{1} y(1)
$$

where $r_{1}$ and $r_{2}$ are the amounts of censoring. Tables of the covarlances of the estimates of location and scale parameters are glven. Dixon (5), in studying the normal distribution, suggests the estimate 


$$
\hat{m}=\left(\alpha x_{(1)}+x_{(2)}+\ldots+(r+1) x_{(n-r)}\right) /(n+2-1) \quad(1.13)
$$

where $a$ is chosen to make the estimate unbiased and $r$ is number of observations censored. Note that Equation 1.13 is used only for censoring the large observations even though the distribution is symmetric.

The estimator above is similar to those suggested by Epstein and Sobel (14, 15) for life testing situations. A life test is an experiment in which the observations occur in order and the experiment is terminated after the $(n-r)-t h$ faliure or at some particular time, or some combination of both. Initially they studied the one parameter exponential distribution and gave the estimator for $b$

$$
\hat{E}_{r}=\left(\sum_{1=1}^{n-r} x_{(1)}+r x_{(n-r)}\right) /(n-r) .
$$

The probability density function of the first $n-r$ of $n$ ordered observations is

$$
f\left(x_{(1)}, x_{(2)}, \ldots, x_{(n-r)} ; b\right)=\frac{n !}{r !} \frac{1}{b^{n-r}} \exp \left(-\left(\sum_{1=1}^{n-r} x_{(1)}+r x_{(r)}\right)^{2 b}\right)
$$

and $\hat{E}_{r}$ maximizes Equation 1.15. Hence $\hat{E}_{r}$ is the maximum likelihood estimate (MLE) for $b$. The estimate $\hat{E}_{r}$ has the same precision as the mean based on a sample of $31 z c(n-r)$. In Chapter IV we shall show that $\hat{E}_{r}$ is unblased. Let us compute the Cramér-Rao lower bound $1 / E(\lambda \log f / \partial \theta)^{2}$, where $f$ is given by Equation 1.15. We write Equation 1.15 as 


$$
\begin{aligned}
& f(x(1), x(2), \ldots, x(n-r) ; b)=\frac{n !}{r ! b^{n-r}} \exp \left(-(n-r) \hat{E}_{r} / b\right) \\
& \log f=\log (n !)-\log (r !)-(n-r) \log (b)-(n-r) \hat{E}_{r} / b \\
& \frac{\partial \log (f)}{\partial b}=-\frac{(n-r)}{b}+\frac{(n-r) \hat{E}_{r}}{b^{2}} .
\end{aligned}
$$

Hence

$$
\begin{aligned}
E\left(\frac{\partial \log f}{\partial b}\right)^{2} & =E\left(-\frac{(n-r)}{b}+\frac{(n-r)}{b^{2}} \hat{E}_{r}\right)^{2} \\
& =(n-r)^{2} E\left(\frac{1}{b^{2}}+\frac{\hat{E}_{r}^{2}}{b^{4}}-\frac{2 \hat{E}_{r}}{b^{3}}\right) \\
& =(n-r) / b^{2}
\end{aligned}
$$

Therefore the Cramér-Rao lower bound is $b^{2} /(n-r)$. But as will be shown in chapter IV, the variance of the estimator is $b^{2} /(n-r)$. Hence $\hat{E}_{r}$ is minimum variance and efficient since any other unbiased estimate based on the first $n-r$ of $n$ order statistics has variance at least $b^{2} /(n-r)$. Using the random variable $z_{1}$ defined in Equation 1.1 we write the estimate as

$$
\hat{\mathrm{E}}_{r}=\sum_{1=1}^{n-r} z_{1} /(n-r)
$$

where the observations $z_{1}$ are independent exponentials with parameter $b$. The characteristic function of the $z_{1}$ is

$$
g_{Z_{1}}(t)=(1-1 t b)^{-1}
$$


hence the quantity $p=2(n-r) \hat{E}_{r} / b$ has characteristic function

$$
g_{p}(t)=(1-12 t)^{-(n-r)}
$$

which is a chi-square variate with $2(n-r)$ degrees of freedom. Epstein and Sobel's second paper (15) discusses a life test to be terminated at the $(n-r)$-th fallure or a preset time, $T$, where the underlying distribution is exponential. Epstein (11) Investigated the two parameter exponential which has distribution function $F(x)=1-e^{-(x-A) / D}, x>A$, and suggested as an estimate of the location parameter $A$

$$
\hat{A}=x(1)-\hat{E}_{r} / n
$$

and developed an $F$ test for $A$.

Bartholomew (1) studied the exponential distribution and estimated the parameter by

$$
\hat{b}=\frac{1}{k} \sum_{1=1}^{n}\left(a_{1} t_{1}+\left(1-a_{1}\right) T_{1}\right)
$$

where the $T_{1}$ are prechosen fallure times for the 1-th exper1ment. The observations $t_{1}$ are known if and only if they fail before their termination time $T_{1}$. Hence $t_{1} \leq T_{1}$. Finaliy $a_{1}=1$ if $t_{1} \leq T_{1}$ and $a_{1}=0$ otherwise, and $k=$ $\sum a_{1}$. Then the $t_{1}$ are not order statistics in the strictest sense. The usual case would be for $T_{1}=T$ for all 1. The estimate is asymptotically normally distributed. Searles (39) extended this estimate to more general distributions. 
Jackson (24) developed a test to measure departures from the exponential. Letting $e_{1}$ be the expected value of the 1 -th standard exponential order statistics, he suggests as a test statistic $T$ where

$$
T=\Sigma e_{1} x_{(1)} / \Sigma \times(1)
$$

It is easily seen that. $T$ is independent of the parameter $b$. He used the results of Renyl to write $T$ as

$$
T=\Sigma c_{1} z_{1} / \Sigma z_{1}
$$

where $c_{1}=1$ and $c_{1}=1+e_{1-1}$ for $1=2,3, \ldots, n$. He finds the distribution function of the test as

$$
F(x)=\sum_{k}\left(\left(x-c_{k}\right)^{n-1} \substack{j=1 \\ j=1 \\ j \neq k}_{\substack{n \\ j \neq k}}\left(c_{j}-c_{k}\right)\right.
$$

which is dependent on the sample size. Furthermore, for sample sizes greater than 30 a normal approximation is satisfactory and $T$ is asymptotically normal with mean two and variance $n^{-1}$. Jackson presents the results of a Monte Carlo study giving the empirical power of the $T$ test testing $H_{O}: f(x)=e^{-x}$ versus the alternative $H_{1}: f(x)=x e^{-x}$. At the five percent level this power is .40 for a sample of size 10 and .56 for a sample of size 20 . He suggests that similar results would occur for other alternatives. The test statistic is expanded to include the censored case. He uses the estimate $\hat{E}_{r}$ and the statistic 


$$
T_{r}=\stackrel{n-r}{\Sigma} e_{1} x_{(1)} \hat{E_{r}}
$$

We shall discuss a similar test in a later chapter.

Harter and Moore. (23) used the estimator $\hat{\mathrm{E}}_{\mathrm{r}}$ for est1mating the scale parameter of type II extreme value distributions. If $T$ is an exponential random variable with parameter $b$ then $Y=T^{-1 / k}$ has type II asymptotic distribution for largest values with scale parameter $v_{1}=b^{-1 / k}$, shape parameter:k> and distribution function given by $F_{1}\left(y ; v_{1}, k\right)=\exp \left(-\left(y / v_{1}\right)^{-k}\right)$. The MIE of $v_{1}$, if the shape parameter is known, is

$$
\begin{aligned}
\hat{v}_{1} & =\left(\hat{E}_{r}\right)^{-1 / k}=\left(\left(\sum^{n-r} t(1)+r t(r)\right) /(n-r)\right)^{-1 / k} \\
& =\left((n-r) \sum_{1=1}^{n-r} y_{(n-1)}^{-k}+r y_{(r)}^{-k}\right)^{1 / k} .
\end{aligned}
$$

The type II asymptotic distribution for smallest values is derived from the exponential by the transformation $\mathrm{x}=$ $-T^{-1 / k}$ and has distribution function $F_{2}\left(x ; v_{2}, k\right)=1-$ $\exp \left(-\left(x / v_{2}\right)^{-k}\right)$. The estimator in this case is derived by the symmetry of the distribution of largest values and hence $v_{1}=-v_{2}$. The estimate of $v_{2} 1 \mathrm{~s}$

$$
\left.\hat{v}_{2}=-\left((n-r) /^{n-r} \Sigma\left(-x_{(1)}\right)^{-k}+r(x(n-r))^{-k}\right)\right)^{1 / k}
$$

The estimators $\hat{v}_{1}$ and $\hat{v}_{2}$ are MIE since they are one-to-one functions of the MIE $\hat{E}_{r}$. Somewhat earlier Harter and 
Moore (23) had considered the Welbull distribution with a known shape parameter. From Equation 1.7 let $\mathrm{X}=\mathrm{T}^{1 / a}$, then the random variable $X$ is distributed exponentially with parameter $c^{1 / a}$. Hence Epstein's estimator $\hat{E}_{r}=\frac{l}{n-r} l^{n-r} x_{(1)}+$ $r x(n-r)$ ), based on the transformed variables estimates $c^{1 / a}$. Therefore $\left(\hat{E}_{r}\right)^{a}$ is the maximum likelihood estimate of c. An unblased estimate for $c$ based on $\hat{E}_{r}$ is $\hat{c}=$ $\left((n-r)^{a} \Gamma(n-r) / \Gamma(n-r+a)\right) \hat{E}_{r}$.

A similar type estimator developed by Charles Winsor and studied by Tukey w1th McLaughlin (43) and Dixon (6) is called the Winsorized mean. This estimate censors the $\mathbf{s}$ smallest and the $r$ largest observations and replaces them with $x_{(s+1)}$ and $x_{(r+1)}$ respectively. Then

$\hat{s}_{r}=\frac{1}{n}\left((s+1) x_{(s+1)}+x_{(s+2)}+\cdots x_{(n-r-1)}+(r+1) x_{(n-r)}\right)$. Tukey, Mclaughlin, and D1xon studied $\hat{s}_{\mathrm{r}}$ for symmetric distributions, primarily the normal distribution. For symmetric Winsorization a t-type test was developed.

$$
t^{*}=\left(\hat{w}_{r}-m\right) / \sqrt{\Sigma\left(x(1)-\hat{w}_{r}\right)^{2} / n(n-1)}
$$

where $m$ is the hypothesized center of the symmetric distribution. They suggest no criterion for preselecting the amount of censoring, rather they suggest the comparison of several amounts of censoring with the "best" result being chosen. The comparison procedure assumes that all of the 
observations are avaliable.

For skewed distributions we shall investigate censoring from only the long talled side. In particular, for the Welbull, we conslder the Winsorlzed mean ${ }_{O} \hat{W}_{r}$ which we shall write as $\hat{W}_{r}$. Then

$$
\hat{w}_{r}=\frac{1}{n}\left(\sum_{1=1}^{n-r} x_{(1)}+r x_{(n-r)}\right) \text {. }
$$

Note that $\hat{W}_{r}$ is related to $\hat{E}_{r}$ by $\hat{W}_{r}=\frac{n-r}{n} \hat{E}_{r}$.

Splitstone's thesis (40) reiterates the graphical techniques for estimation in the Welbull as well as other approaches on an easily understood level. He and many others have investigated a transformation technique in which the logarithms of the observations are taken. If we write the Welbull density as

$$
f(t ; a, b)=\frac{t^{\frac{1}{a}-1}}{a c^{1} / a^{\exp }\left(-(t / c)^{1 / a}\right)}
$$

and let

$$
\begin{aligned}
& \mathrm{X}=\log \mathrm{T} \\
& \mathrm{c}^{1}=\log \mathrm{c}
\end{aligned}
$$

where $T$ is the Welbull random varlable then $X$ is distributed as the extreme value distribution with density function

$$
h\left(x ; a, c^{1}\right)=\frac{1}{a} \exp \left(\frac{x-c^{1}}{a}\right) \exp \left(-\exp \left(\frac{x-c^{1}}{a}\right)\right)
$$


It is seen that the parameters of $h$ are of the location and scale types. Lleblein and Zelen (26) derlve welghts for the order statistics which give best linear unbiased estimates of the parameters of the extreme value distribution for both censored and noncensored samples up to size six. White (47) uses Lloyd's least square procedure to derive estimators

$$
\hat{a}=\stackrel{n-r}{\Sigma} a_{1} x(1)
$$

and

$$
\hat{c^{1}}=\stackrel{n-r}{\Sigma} b_{1} x(1)
$$

for censored samples w1th $n=0, \ldots, n-1$ and gives the coefficients for samples up to size 20. Tables of the expected values and covariances of these estimators are given. Downton (8) also uses the logarithmic transformation of the Weibull and notes that

$$
E(\log T)=c^{1}-8 a
$$

and

$$
V(\log T)=\frac{\pi}{6}^{2} a^{2}
$$

where of is Euler's constant. He further suggests an estimate based on the original data. He defines random variables $V$ and $W$ with realizations $v$ and $w$ such that

$$
v=\sum^{n} t(1)
$$


and

$$
w=\sum 1 t(1)
$$

where $t_{(1)}$ are the order statistics of the weibull. He arrives at unbiased estimates

$$
\hat{a}=-\frac{n+1}{n(n-1)^{10 g} 2} v+\frac{2}{n(n-1) \log 2} w
$$

and

$$
\hat{c}=\frac{(n-1) \log 2-(n+1)}{n(n-1) \log 2} \delta v+\frac{2 \delta}{n(n-1) \log 2} w
$$

and computes the covarlances of the estimates. Downton was not able to show asymptotic normality of his estimates. Finaily, as a single source, Mann (30) provides a short discourse on most methods of estimation of the parameters of the Weibull and a very complete bibllography. 
CHAPTER II. WEIBULL ORDER STATISTICS

A. Expected Moments of Welbull Order Statistics Consider the Welbull density of the form of Equation 1.11

$$
\begin{aligned}
f(y ; a, b) & =\frac{1}{a b} y^{\frac{1}{a}-1} \exp \left(-y^{1 / a} / b\right), y>0, b>0, a>0 \\
& =0 \text { otherwise. }
\end{aligned}
$$

The transformation $\mathrm{X}=\mathrm{Y}^{1 / a}$ is one to one, order preserving, and the random variable $x$ is distributed as an exponential with parameter $b$. Then if $Y_{(1)}$ are the order statistics drawn from the distribution of Equation 2.1, $X_{(1)}=Y_{(1)^{1 / a}}$. We have previously shown in the literature review that

$$
\begin{aligned}
& z_{k}=(n-k+1)\left(x_{(k)}-x_{(k-1)}\right) \\
& k=1,2, \ldots, n
\end{aligned}
$$

are independent and identically distributed as exponential with parameter b. Furthermore, $z_{1}$ is independent of $x_{(j)}$ if 1 is greater than $j$. Now the moment generating function for the exponential is given by

$$
\mathrm{mgf}_{z_{1}}(t)=(1-t b)^{-1} \text {. }
$$

The $m$-th moment of the exponential may be found by taking derivatives with respect to $t$. 


$$
E\left(z_{1}^{m}\right)=\Gamma(m+1) b^{m}
$$

where $\Gamma$ is the Gamma function. Suppose we set $b$ equal to unity and rewrite Equation 2.2 as

$$
x_{(k)}=x_{(k-1)}+z_{k} /(n-k+1) .
$$

From Equation 2.5 since $x_{(0)}=0$

$$
E\left(x_{(1)}^{\mathrm{m}}\right)=E\left(\left(z_{1} / n\right)^{\mathrm{m}}\right)
$$

Now evaluating the right-hand side of Equation 2.6 using Equation 2.4 we have for $m$ a positive integer.

$$
E\left(X_{(1)}^{m}\right)=m !\left(\frac{l}{n}\right)^{m}
$$

Continuing and using the binomial theorem we have

$$
\begin{aligned}
E\left(X_{(2)}^{m}\right) & =E\left(\left(X(1)+z_{2} /(n-1)\right)^{m}\right) \\
& =E\left(\sum_{1=0}^{m}\left(m_{1}^{m}\right) X_{(1)}^{1}\left(z_{2} /(n-1)\right)^{m-1}\right)
\end{aligned}
$$

but $x_{(1)}$ and $z_{2}$ are independent so we have

$$
E\left(x_{(2)}^{m}\right)=\sum_{1=0}^{m}\left(\begin{array}{l}
m \\
1
\end{array}\right) E\left(x_{(1)}^{1}\right) E\left(\left(z_{2} /(n-1)^{m}\right) .\right.
$$

Now using Equations 2.6 and 2.7 we have

$$
E\left(x_{(2)}^{m}\right)=m ! \sum_{1=0}^{m}\left(\frac{1}{n}\right)^{1}\left(\frac{1}{n-1}\right)^{m-1} .
$$

Assume that 


$$
\begin{aligned}
& E\left(X_{(k)}^{m}\right)=m ! \sum_{i_{1}=0}^{m} \sum_{1_{2}=0}^{m-1_{1}} \ldots \sum_{i_{k-1}=0}^{\left(m-\sum_{j=1}^{k-2} 1_{j}\right.}\left(\frac{1}{n}\right)^{1} l\left(\frac{1}{n-1}\right)^{1_{2}} \ldots \\
& \left(\frac{1}{n-k+2}\right)^{1} k-1\left(\frac{\lambda}{n-k+1}\right)^{m-k-1} \sum_{j=1}^{1} j \text {. }
\end{aligned}
$$

Let $S(n, k, m)$ be the sum on the right hand side of 2.9. Hence $E\left(X_{(k)}^{m}\right)=m ! S(n, k, m)$. Now, using the assumption of 2.9 , we find the expected moments of the $(k+1)$-st order statistic.

$$
\begin{aligned}
& E\left(X_{(k+1)}^{m}\right)=E\left(\left(x_{(k)}+\frac{Z_{k+1}}{n-k}\right)^{m}\right) \\
& =\sum_{1=0}^{m}\left(\begin{array}{l}
m \\
1
\end{array}\right) E\left(\left(\frac{{ }^{2} k+1}{n-k}\right)^{1}\right) E\left(x_{(k)}^{m-1}\right) \\
& =\sum_{1=0}^{m} \frac{m !}{1 !(m-1) !}{ }^{1 !}\left(\frac{1}{n-k}\right)^{1} E\left(x(k)^{m-1}\right) \\
& E\left(x_{(k+1)}^{m}\right)=m ! \sum_{1=0}^{m}\left(\frac{1}{n-k}\right)^{1} E\left(x(k)^{m-1}\right) /(m-1) ! \\
& =m ! \sum_{1=0}^{m} \frac{1}{(m-1) !}\left(\frac{1}{n-k}\right)^{1}(m-1) ! s(n, k, m-1) \\
& =m ! \sum_{1=0}^{m}\left(\frac{1}{n-k}\right)^{1} s(n, k, m-1) \\
& =m ! s(n, k+1, m)
\end{aligned}
$$

which is the desired result. Hence Equation 2.9 is the general formula for computing a particular expected value. For evaluation of an entire table for $k=1,2, \ldots n$, 
1t is computationally more efficient to use the relationship from Equation 2.10a

$$
E(X(k))=m ! \prod_{1=0}^{m} E\left(X\left(k_{k-1}^{1}\right)\left(\frac{1}{n-k+1}\right)^{m-1} / 1 !\right.
$$

Equation 2.11 has been eveluated for $n=1,2, \ldots, 25 ; k=$ $1,2, \ldots, n$; and $m=1,2, \ldots, 10$ by McElhone and Larsen In (28) and a portion of that table appears here as Table 1. The table. 1s used in the following manner. Recall that if $Y$ is a Weibull random variable with shape parameter a,

$$
E\left(Y_{(k)}^{q}\right)=E\left(X_{(k)}^{a q}\right)
$$

where $X$ is an exponential random variable. Hence the value of $E\left(Y_{(k)}^{q}\right)$ is found in the aq-th column of the table. Because of space restrictions, the sixth through the tenth columns appear directly below the first through fifth columns. For example, the value of the second moment of the third order statistic of a Welbull with shape parameter two from a sample of size five is 1.7871. If the shape parameter were five in the above case the value would be $5.64318+02=564.31$.

Relaxing the condition that $b$ be unity, it is seen that for X's distributed exponentially with parameter $b$, the moments are given by

$$
E\left(X_{(k)}^{m}\right)=m ! b^{m_{S}}(n, k, m)
$$

Hence the values of the q-th moment of a weibull with shape 
TABLE 1 .

Moments of the $K-t h$ order statistic of a sample of size $N$ from the exponential distribution

$\mathrm{E}\left(\mathrm{X}_{(\mathrm{K})}^{\mathrm{M}}\right)$

$\mathrm{N}=2$

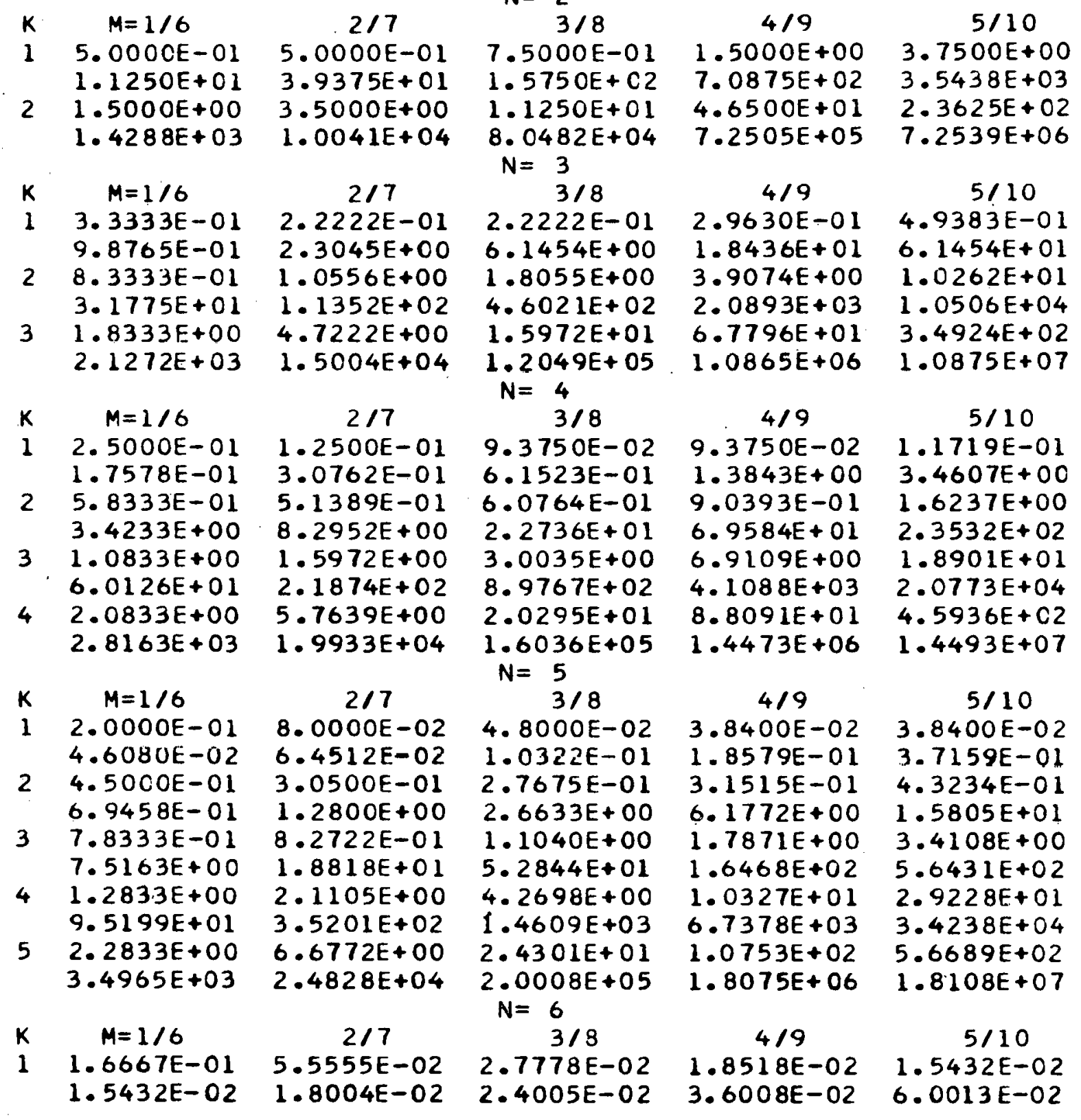


TABLE 1 - (CONT INUED)

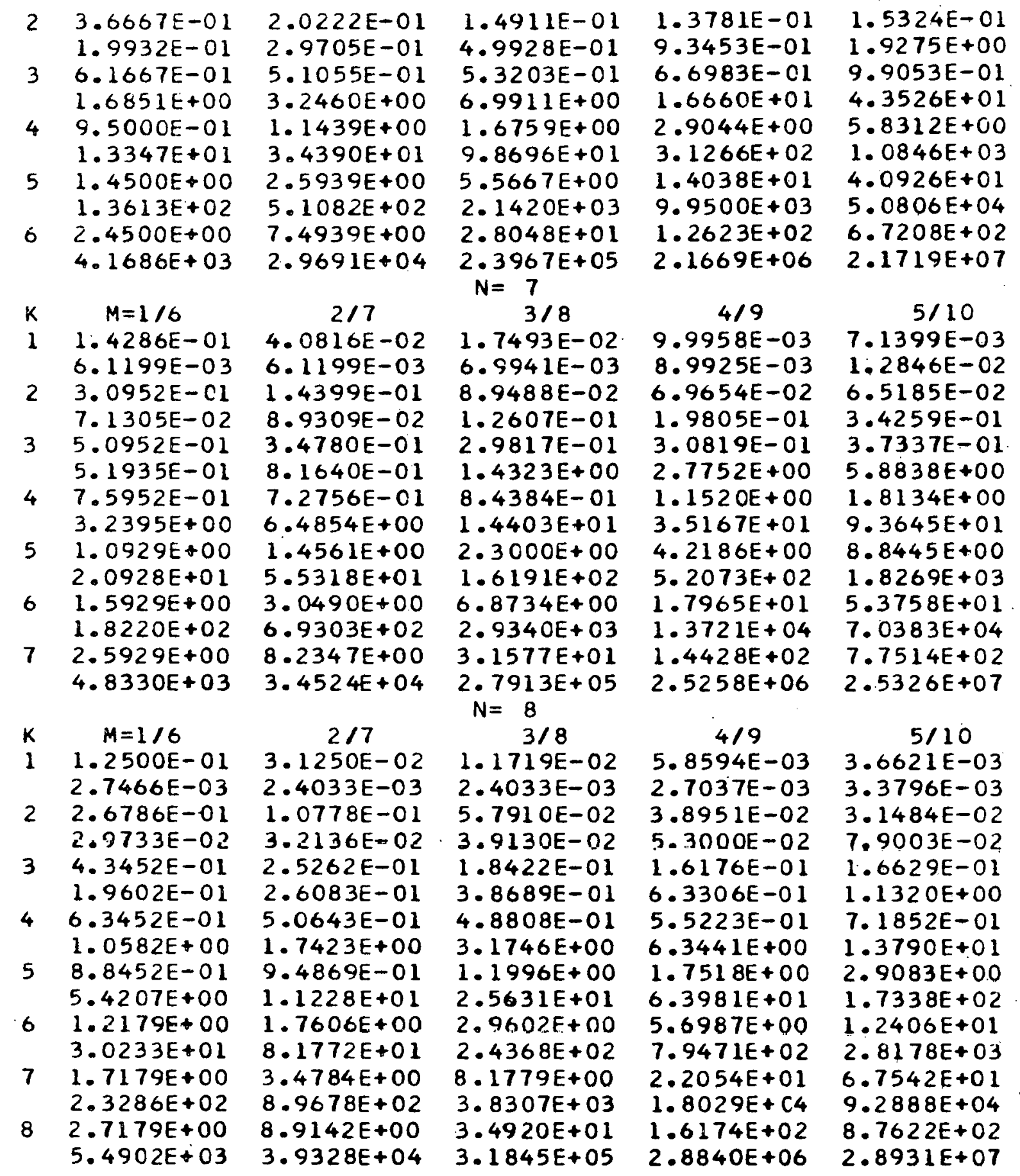


TABLE 1. (CONT INUED)

\begin{tabular}{|c|c|c|c|c|c|}
\hline & & & $N=$ & & \\
\hline$k$ & $M=1 / 6$ & $2 / 7$ & $3 / 8$ & $4 / 9$ & $5 / 10$ \\
\hline 1 & $\begin{array}{l}1.1111 \mathrm{E}-01 \\
1.3548 \mathrm{E}-03\end{array}$ & $\begin{array}{l}2.4691 E-02 \\
1.0537 E-03\end{array}$ & $\begin{array}{l}8.2304 E-03 \\
9.3665 E-04\end{array}$ & $\begin{array}{l}3.6580 E-03 \\
9.3665 E-04\end{array}$ & $\begin{array}{l}2.0322 E-03 \\
1.0407 E-03\end{array}$ \\
\hline 2 & $2.3611 E-01$ & $8.3719 E-02$ & $3.9625 E-02$ & $2.3470 E-02$ & $1.6701 E-02$ \\
\hline & $1.3881 E-02$ & $1.3199 E-02$ & $1.4136 E-02$ & $1.6835 \mathrm{E}-02$ & $2.2056 E-02$ \\
\hline 3 & $3.7897 E-01$ & $1.9200 E-01$ & $1.2191 E-01$ & $9.3133 \mathrm{E}-02$ & $8.3224 E-02$ \\
\hline & $6 E-02$ & $9.8415 E-02$ & $1.2661 E-01$ & $3 E-01$ & $2.7794 E-01$ \\
\hline 4 & $5.4563 E-01$ & $3.7387 E-01$ & $3.0885 E-01$ & $3 E-01$ & 3. $3242 \mathrm{E}-01$ \\
\hline & 4.17 & $5.8565 E-01$ & 9.0 & 1.53 & $3 E+00$ \\
\hline 5 & 7.45 & $6.7213 E-01$ & $7.1212 E-01$ & $E-01$ & $E+00$ \\
\hline & 1.85 & $3.1882 E+00$ & $6.0084 E+00$ & $7 E+01$ & 2.7 \\
\hline 6 & $E-01$ & $1.1699 E+00$ & $1 \cdot 5$ & $83 E+00$ & 4.2 \\
\hline & $E+00$ & $1.7661 E+01$ & $4.1328 E+01$ & 1.05 & $2 \cdot 8$ \\
\hline 7 & $1 \cdot 32$ & 2.05 & $3 \cdot 6$ & 7.3 & 1.6 \\
\hline & 4. & 12 & $\begin{array}{l}3.4 \\
9.4\end{array}$ & 3 & $\begin{array}{l}4.0799 E+03 \\
8.2134 E+01\end{array}$ \\
\hline 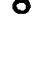 & $\begin{array}{l}1.8290 E+00 \\
2.8761 E+02\end{array}$ & $1.1205 E+03$ & $4.8267 E+03$ & $2.2854 E+04$ & $1.1824 E+05$ \\
\hline 9 & $2.8290 E+00$ & $8 E+00$ & 3.81 & .02 & $9.7548 E+02$ \\
\hline & & & $\begin{array}{l}3.5766 E+05 \\
N=10\end{array}$ & & \\
\hline$k$ & $M=1 / 6$ & $2 / 7$ & $3 / 8$ & $4 / 9$ & $5 / 10$ \\
\hline 1 & $E-01$ & $2.0000 E-02$ & $6.0000 E-03$ & $2.4000 E-03$ & $1.2000 E-03$ \\
\hline & & 04 & & 3.62 & 04 \\
\hline 2 & $1 E-01$ & $6.6913 E-02$ & $2.8304 E-02$ & $1.4980 \mathrm{E}-02$ & $9.5221 E-03$ \\
\hline & 7.0 & 6.00 & $5.7376 E-03$ & 6.09 & 7.129 \\
\hline 3 & $E-01$ & 1.50 & $7 E-02$ & $7433 E-02$ & 4.5 \\
\hline & -02 & $1 E-02$ & $4.7727 E-02$ & $5.9761 E-02$ & $8.1641 E-02$ \\
\hline 4 & $E-01$ & 2.8 & $E-C 1$ & $1.7643 E-01$ & $44 E-01$ \\
\hline & $E-01$ & 2 & 1 & 4.58 & $99 E-01$ \\
\hline 5 & $6.4563 E-01$ & $5.0300 E-01$ & $E-01$ & $4.8293 E-01$ & $5.7388 E-01$ \\
\hline & 7.61 & 1.119 & 1. $8 \cap>G F$ & 3.16 & $5.9828 E$ \\
\hline 6 & $8.4563 E-01$ & $8.4125 E-01$ & $9.6450 E-01$ & $1.2545 E+00$ & $1.8284 E+00$ \\
\hline & 2.95 & $2574 E+00$ & $1.0214 E+$ & 2.1530 & $4.8883 E+01$ \\
\hline 7 & $1.0956 E+00$ & $1.3891 E+00$ & $2.0063 E+00$ & $3.2608 E+00$ & $5.9044 E+00$ \\
\hline & $1.1813 E+01$ & $2.5929 E+01$ & $2070 E+$ & 610 & 4. $5028 E+02$ \\
\hline 8 & $1.4290 E+00$ & $2.3417 E+00$ & $4.3480 E+00$ & $9.0582 E+00$ & $2.1001 E+01$ \\
\hline & $5.3815 E+01$ & $1.5150 E+02$ & $4.6605 E+02$ & $1.5584 E+03$ & $5.6330 E+03$ \\
\hline 9 & $1.9290 E+00$ & $4.2707 E+00$ & $1.0754 E+01$ & $3.0566 E+01$ & $9.7417 E+01$ \\
\hline & rover & $E+03$ & $168 E+03$ & $6 E+04$ & $1.4636 E+05$ \\
\hline 10 & $2.9290 E+00$ & $1.0129 E+01$ & $4.1140 E+01$ & $1.9513 E+02$ & $1.0730 E+03$ \\
\hline & $843 E+03$ & $3 E+04$ & $4 E+05$ & $5987 E+06$ & $6129 E+07$ \\
\hline
\end{tabular}


parameter $a$ and scale parameter $b$ is found by multiplying the associated unit scale tabular value by $b^{\text {aq }}$.

The closed form for the expected values of exponential order statistics is known and is given in Equation 4.5. Our computed values for the exponential are in the first column of Table 1. The tabular values agree to the fifth significant digit with the values calculated from Equation 4.5 .

From Equations 2.12 and 2.13

$$
\begin{aligned}
& E\left(Y(k)=a ! b^{a} S(n, k, a)\right. \\
& E\left(Y(k)^{2}\right)=(2 a) ! b^{2 a} S(n, k, 2 a) .
\end{aligned}
$$

The varlance is then

$$
V(Y(k))=b^{2 a}\left((2 a) ! S(n, k, 2 a)-(a !)^{2} s^{2}(n, k, a)\right) .
$$

In terms of the exponential variates we have

$$
V\left(Y_{(k)}\right)=E\left(X_{(k)}^{2 a}\right)-E^{2}\left(X_{(k)}^{a}\right) \text {. }
$$

Now from Equation 1.10 unordered Welbull variates have expectation

$$
E\left(Y^{m}\right)=(a m) ! b^{a m}
$$

so that

$$
E\left(Y_{(k)}^{m}\right)=E\left(Y_{j}^{m}\right) S(n, k, a m)
$$


B. Expected Crossproducts of Weibull order Statistics

From Equation 2.5 we write, for $\mathrm{J} k$

$x_{(k)}=x_{(k-1)}+\frac{z_{k}}{n-k+1}$

$x_{(k)}=x_{(k-2)}+\frac{z_{k-1}}{n-k+2}+\frac{z_{k}}{n-k+1}$

$x_{(k)}=x_{(k-j)}+\sum_{1=1}^{j} \frac{z_{k-1+1}}{n-k+1}$.

Let $w_{k, j}=\sum_{1=1}^{j} z_{k-1+1} /(n-k+1)$. Then

$$
\begin{aligned}
& x_{(k)}=x_{(k-j)}+w_{k, j} \\
& w_{k, j}=w_{k, j-1}+\frac{z_{k-j+1}}{n-k+j} \\
& w_{k, 0}=0 .
\end{aligned}
$$

Taking the expected values of the $m$-th power of Equation 2.20 using the binomial theorem

$E\left(w_{k, j}^{m}\right)=m ! \sum_{1=0}^{m} E\left(w_{k, j-1}\right)\left(\frac{1}{n-k+j}\right)^{m-1} / 1 !$

We are now able to generate the moments of the crossproducts by using Equation 2.19. In fact the computational formula $1 s(2.22)$. 


$$
\begin{aligned}
E\left(\left(x_{(k-j)} x_{(k)}\right)^{m}\right) & =E\left(\left(x_{(k-j)}\left(x_{(k-j)}+w_{k, j}\right)\right)^{m}\right) \\
& =E\left(\left(x_{(k-j)}^{2}+x_{(k-j)} w_{k, j}\right)^{m}\right) . \\
& =\sum_{1=0}^{m}\left(\begin{array}{c}
m \\
1
\end{array}\right) E\left(x_{(k-j)}^{m+1}\right) E\left(w_{k, j}^{m-1}\right) .
\end{aligned}
$$

The terms in the summation are precalculated from Equations 2.9 and 2.21. For b not unity, multiply the right-hand side of Equation 2.22 by $b^{m}$. From the above and Equation 2.9, we have the covarlances as

$$
\operatorname{Cov}\left(x_{(k)}^{p} X_{(j)}^{p}\right)=E\left(\left(X_{(k)} x_{(j)}\right)^{p}\right)-E\left(X_{(k)}^{p}\right) E\left(X_{(j)}^{p}\right) .
$$

Equation 2.23 has been evaluated for the same values of $n$ and $k$ as the expected value table. The entire table is presented in (28) and a portion of it appears here as Table 2. The covariance of a Welbull varlate with shape parameter a is found In column a of the table. For example, if a equals three then $C\left(Y_{(1)}, Y_{(3)}\right)$ for a sample of size flve is 0.14866 .

The closed form for the covariances of exponential order statistics is known and is given in Equation 4.6. Our computed values for the exponential are in the first column of Table 2. When compared to the exact results, our results show some small error in the fifth digit. The 
TABLE 2 .

Covariances of the $\mathrm{K}$-th and $\mathrm{J}$-th order statistics of a sample of size $\mathrm{N}$ from the Weibull distribution w1th shape parameter $M$

$$
\mathrm{C}\left(\mathrm{Y}_{(\mathrm{K})}, \mathrm{Y}_{(\mathrm{J})}\right)
$$

\section{Shape parameter}

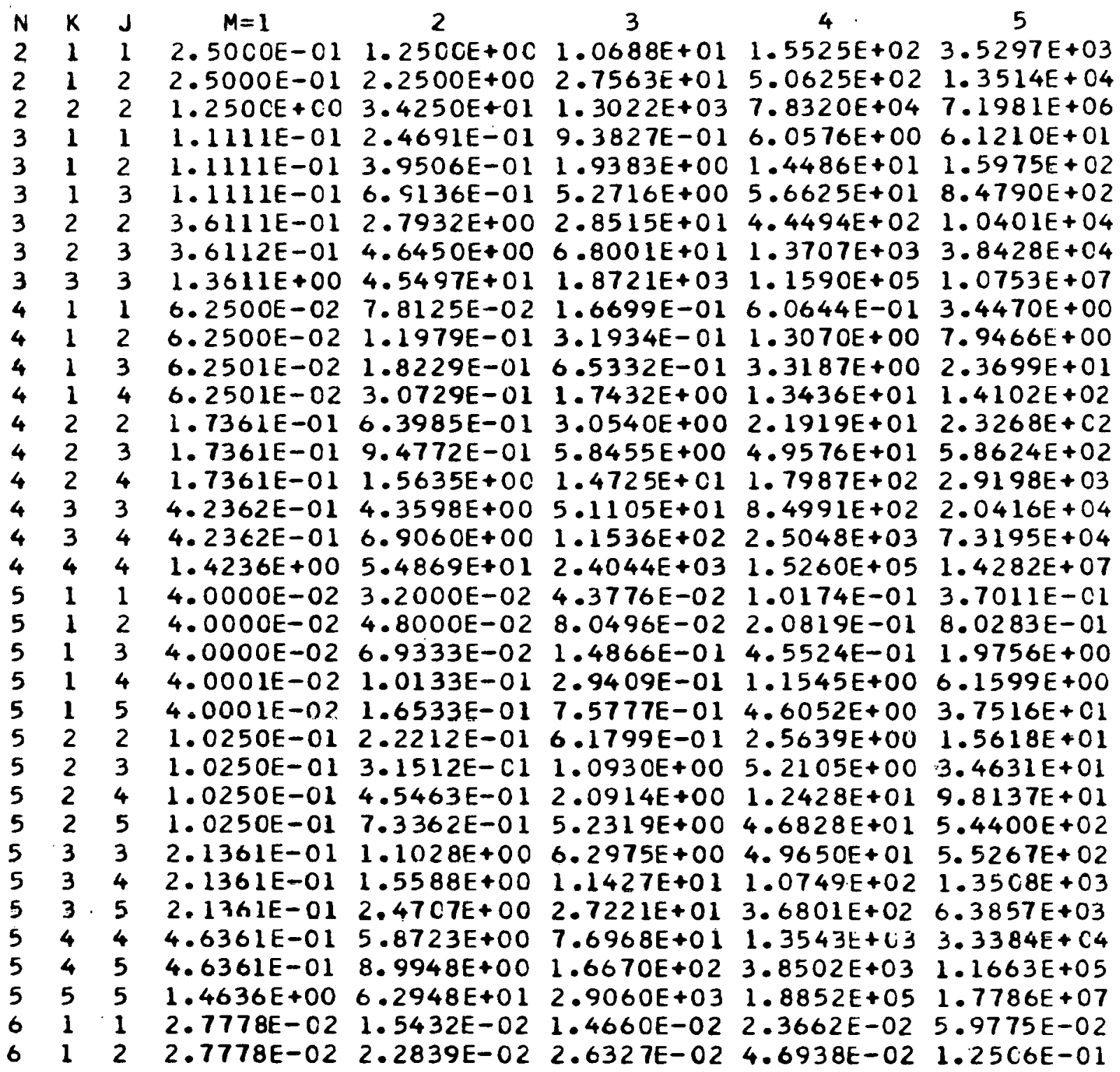


TABLE 2. (CONTINUED)

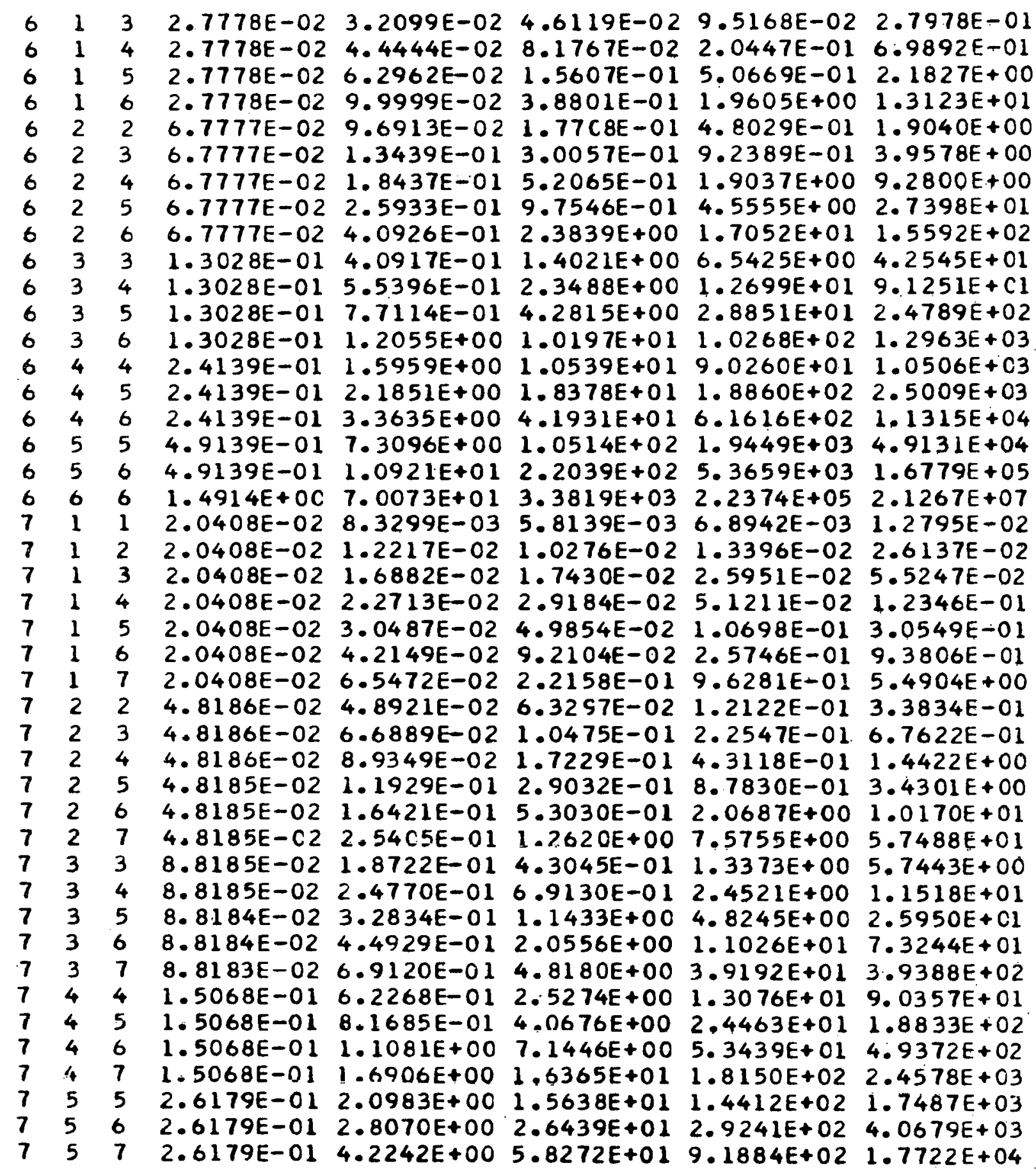


TABLE 2. (CONT INUED)

$\begin{array}{lll}7 & 6 & 6 \\ 7 & 6 & 7 \\ 7 & 7 & 7 \\ 8 & 1 & 1 \\ 8 & 1 & 2 \\ 8 & 1 & 3 \\ 8 & 1 & 4 \\ 8 & 1 & 5 \\ 8 & 1 & 6 \\ 8 & 1 & 7 \\ 8 & 1 & 8 \\ 8 & 2 & 2 \\ 8 & 2 & 3 \\ 8 & 2 & 4 \\ 8 & 2 & 5 \\ 8 & 2 & 6 \\ 8 & 2 & 7 \\ 8 & 2 & 8 \\ 8 & 3 & 3 \\ 8 & 3 & 4 \\ 8 & 3 & 5 \\ 8 & 3 & 6 \\ 8 & 3 & 7 \\ 8 & 3 & 8 \\ 8 & 4 & 4 \\ 8 & 4 & 5 \\ 8 & 4 & 6 \\ 8 & 4 & 7 \\ 8 & 4 & 8 \\ 8 & 5 & 5 \\ 8 & 5 & 6 \\ 8 & 5 & 7 \\ 8 & 5 & 8 \\ 8 & 6 & 6 \\ 8 & 6 & 7 \\ 8 & 6 & 8 \\ 8 & 7 & 7 \\ 8 & 7 & 8 \\ 8 & 8 & 8 \\ 9 & 1 & 1 \\ 9 & 1 & 2 \\ 9 & 1 & 3 \\ 9 & 1 & 4 \\ 9 & 1 & 5\end{array}$
$5.1179 E-01$
$8.6692 E+00$
$1.3496 E+02$
5.1179E-01
$1.2703 E+012.7546 E+02$
2. $6112 E+03$
$6.7493 E+04$
1. $5118 \mathrm{E}+00$
$7.6466 E+01$
$3.8359 E+03$
$1.0219 E+03$
$2.2590 E+05$
1. 5625E-02
$4.8828 E-03$
$2.6092 E-03$
$2.5831 E+05$
$2.4726 E+07$
1. 5625E-02
$7.1150 E-03$
$4.5599 E-03$
$3689 E-03$
$3.3662 E-03$
$1.5625 \mathrm{E}-02$
9.7191E-03
7. $5680 \mathrm{OE}-03$
(5)
$6.7602 E-03$
$1.5625 E-02$
$1.2844 \mathrm{E}-02$
$1.2232 \mathrm{E}-02$
8. $5228 E-03$
$1.3761 E-02$
1. 5625E-02
1.675 OE-02
$1.9711 E-02$
$1.5971 \mathrm{E}-02$
2. $876 \mathrm{CE}-02$
1. $5625 E-02$
$2.1959 E-02$
3.2612E-02
$3.0541 E-02$
$6.3223 E-02$
$1.5624 \mathrm{E}-02$
$2.9771 E-02$
$5.8555 E-02$
6. $1924 E-02$
$1.5331 E-01$
1. $5624 E-02$
4. $5356 E-02$
1. $3681 E-01$
$1.4469 E-01$
4.5961E-01
$3.6034 E-02$
$2.7334 E-02$
$2.6379 E-02$
$5.2328 E-01$
$2.6063 E+00$
$3.6034 E-02$
$3.7014 E-02$
$4.2907 E-02$
3.7613E-02
7. $8012 E-02$
$3.6033 E-02$
$4.8631 E-02$
$6.8366 E-02$
$3.6033 E-02$
$6.3151 E-02$
1. $0898 E-01$
$6.8384 E-02$
1. $5184 E-01$
$3.6033 E-02$
8.2511E-02
1. $7876 E-01$
3. $0591 \mathrm{E}-01$
3. $6033 E-02$
$1.1155 E-01$
$3.1858 E-01$
$2.3434 E-01$
$6.5237 E-01$
$3.6033 E-02$
$1.6963 E-01$
$7.3886 E-01$
4. $6753 \mathrm{E}-01$
$1.5417 \mathrm{E}+00$
$6.3811 E-02$
$9.7947 E-02$
$1.62 \mathrm{CBE}-01$
$1.0771 E+00$
4. $5161 E+00$
$6.3811 E-02$
$1.2773 E-01$
$2.5354 E-01$
$3.8403 E+00$
$2.5013 E+01$
$6.3811 E-02$
$1.6495 \mathrm{E}-01$
$3.9852 E-01$
3.6072E-0
1. $1043 E+00$
$6.3810 E-02$
$2.1459 E-01$
$6.4629 E-01$
$6.3845 E-01$
$2.1243 E+00$
6. $3810 E-02$
2.8904E-OI
$1.14 \mathrm{C} 5 \mathrm{E}+00$
1. $1668 E+00$
$4.3554 E+00$
$6.3809 E-02$
$4.3794 E-01$
$2.6193 E+00$
1. $0381 \mathrm{E}-01$
$2.9576 E-01$
$8.2002 E-01$
$2.2786 E+00$
$9.9471 E+00$
$5.1510 E+00$
$2.8246 E+C 1$
$1.0381 E-01$
$3.7912 E-01$
$1.2645 E+0 C$
$1.8018 E+01$
1. $5153 E+02$
$2.8696 E+00$
$1.3274 E+01$
$1.0381 E-01$
4. $9028 E-01$
$2.0188 E+00$
$5.0644 E+00$
2. $5812 E+01$
$1.0381 E-01$
6.5702E-01
3. $5140 E+00$
$9.6015 E+00$
$5.6230 E+01$
$1.0381 E-01$
9. $9049 E-01$
$7.9596 E+00$
$2.1136 E+01$
$1.5281 E+02$
$1.6631 E-01$
8.5181E-01
$3.9816 E+00$
$7.1952 E+01$
7. $8305 E+02$
$1.6631 E-01$
1. $0921 E+00$
$6.2124 E+00$
$2.2562 E+01$
$1.6492 \mathrm{E}+02$
$1.6631 E-01$
$1.4526 E+00$
$1.0595 E+01$
4. $0951 E+01$
$3.3530 E+02$
$1.6631 \mathrm{E}=01$
$2.1735 E+00$
$2.3504 E+01$
$8.66 C 4 E+01$
$8.5256 E+C 2$
$2.7742 E-01$
$2.5990 E+00$
$2.1470 E+01$
$2.8280 E+02$
$4.0685 E+03$
2.7742E-01
$3.4151 \mathrm{E}+00$
$3.5402 E+01$
2. $1121 \mathrm{E}+02$
$2.6639 E+03$
2.7742E-01
$5.0471 E+00$
$7.5829 E+01$
4. $1801 E+02$
$6.0716 E+03$
5.2741E-01
$9.9549 E+00$
$1.6598 \mathrm{E}+02$
$1.2708 E+03$
$2.5596 E+04$
$5.2741 E-01$
$1.4360 E+01$
$3.3131 E+02$
$.3443 E+03$
$8.8326 E+04$
1. $5274 E+00$
8. $2274 E+01$
4. $2708 E+03$
$8.7955 E+03$
$2.9032 E+05$
1. $2346 E-C 2$
$3.0483 E-03$
$1.2871 E-03$
2. $9230 E+05$
$2.8163 E+07$
1. 2346E-02
4. $4200 E-03$
2.2301E-03
9.2327E-04
$1.0366 E-03$
$1.2346 E-02$
$5.9877 \mathrm{E}-03$
$3.6439 E-03$
$1.7484 E-03$
$2.0555 E-C 3$
1. $2346 E-02$
7.8167E-03
5.75 C5E-03
3. $2143 E-03$
$4.0736 E-03$
$1.2346 E-02$
$1.0011 E-02$
8. $9368 \mathrm{E}-03$
$5.8170 E-03$
$8.1413 E-03$
$1.0538 E-02$
$1.6659 E-02$ 
TABLE 2. (CONTINUEU)

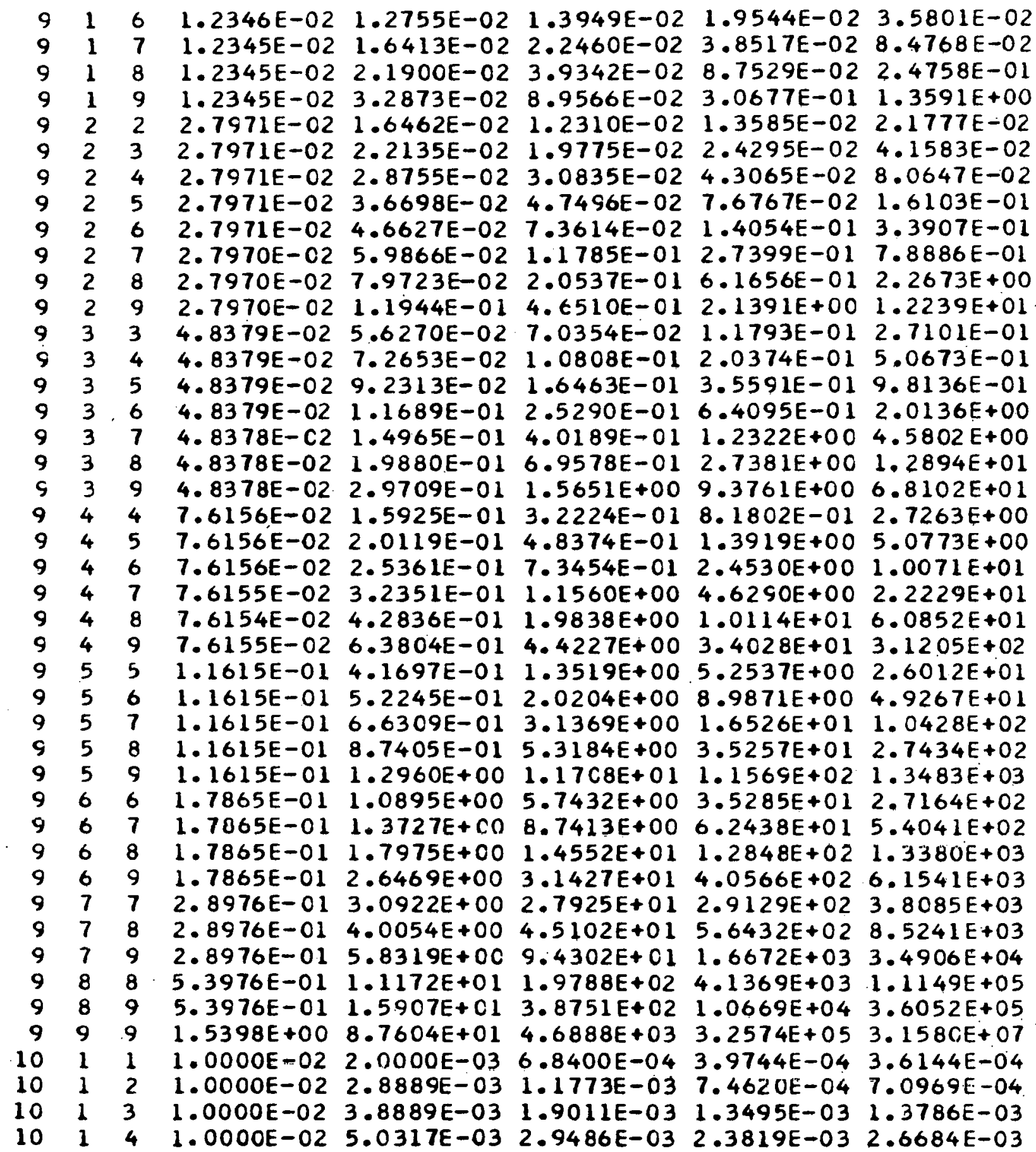


TABLE 2. (CONTINUEO)

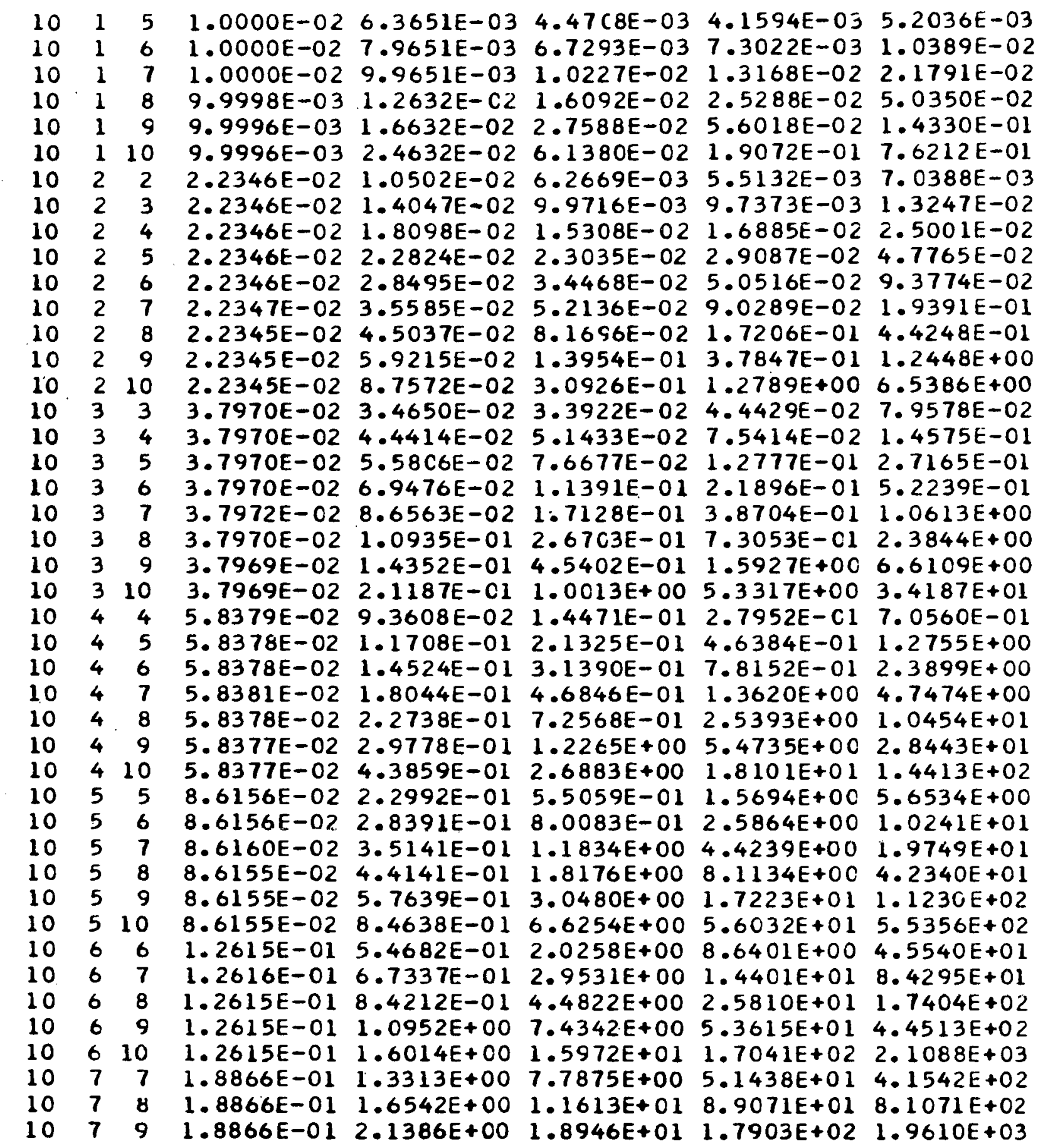


TABLE 2. (CONTINUED)

$\begin{array}{rrrrrrrr}10 & 7 & 10 & 1.8866 E-01 & 3.1074 E+00 & 3.9988 E+01 & 5.4894 E+02 & 8.7301 E+03 \\ 10 & 8 & 8 & 2.9976 E-01 & 3.5746 E+00 & 3.4910 E+01 & 3.8400 E+02 & 5.1919 E+03 \\ 10 & 8 & 9 & 2.9976 E-01 & 4.5763 E+00 & 5.5407 E+01 & 7.3021 E+02 & 1.1432 E+04 \\ 10 & 8 & 10 & 2.9976 E-01 & 6.5800 E+00 & 1.1347 E+02 & 2.1039 E+03 & 4.5614 E+04 \\ 10 & 9 & 9 & 5.4976 E-01 & 1.2327 E+01 & 2.3042 E+02 & 4.9825 E+03 & 1.3687 E+05 \\ 10 & 9 & 10 & 5.4976 E-01 & 1.7359 E+01 & 4.4382 E+02 & 1.2628 E+04 & 4.3603 E+05 \\ 10 & 10 & 10 & 1.5497 E+00 & 9.2537 E+01 & 5.0918 E+03 & 3.5867 E+05 & 3.4978 E+07\end{array}$


computation was executed in single precision and there is evidence of round off error.

Consider the summation in Equation 2.9. The form $S(n, n, m)$, for the largest order statistic, is monotone increasing in $m$. This follows since the difference $s(n, n, k)$ $S(n, n, m-1)$ is made up of the sum of all of the possible products of $(1 / 2)^{1}(1 / 3)^{\frac{12}{2}} \ldots(1 / n)^{\ln -1}$ such that $\Sigma_{1}=m$. Each of these products is less than $(1 / 2)^{\mathrm{m}}$. Now we count the number of these products. We are dealing with $n-1$ fractions. First we count the products made up of a single fraction. There are $\left(\begin{array}{c}n-1 \\ 1\end{array}\right)$ of type $(1 / 1)^{m}$. Now there are $\left(\begin{array}{c}n-1 \\ 2\end{array}\right)$ ways to plck the products of pairs of fractions. There are $2:\left(\frac{n-1}{2}\right)$ products of the type $(1 / 1)^{\mathrm{m}-1}(1 / \mathrm{j})$ since we must permute the power; that 1 , e1ther the $(1 / 1)$ or the $(1 / \mathrm{J})$ may have the power $m-1$. There are also $2 !\left(\begin{array}{c}n-1 \\ 2\end{array}\right)$ products of the type $(1 / 1)^{m-2}(1 / \jmath)^{2}$. The highest power can decrease to $\left[\frac{m}{2}\right]$, where $\left[\frac{m}{i}\right]$ is the largest integer function. Therefore, the number of producto of the type $(1 / 1)^{m-k}(1 / j)^{k}$ is 2 ! $\left[\frac{m}{c}\right]\left(\frac{n-1}{2}\right)$. Now looking at the products of three fractions we see that these number less than or equal to $3 !\left[\frac{m}{3}\right]\left(\frac{n-1}{3}\right)$. This procedure is continued unt1l we count the single product $(1 / 2)(1 / 3) \ldots$ $(1 / n)$. Adding these we see that the total number of products is less than or equal to

$$
n+\sum_{1=2}^{n-2}\left[\frac{m}{1}\right] 1:\left(\begin{array}{c}
n-1 \\
1
\end{array}\right)
$$


Hence $S(n, n, m)-S(n, n, m-1) \leq\left(\frac{1}{2}\right)^{m}\left(n+\sum_{1=2}^{n-2}\left[\frac{m}{1}\right] 1 !\left(\begin{array}{c}n-1 \\ 1\end{array}\right)\right)$ For a fixed $n$ this difference approaches zero as $m$ increases. The values of $S(n, k, m)$ were computed in confunction with Table 1. The values of $S(n, n, m)$ were found to converge rapldiy as the moments increased and became stablized by. $m=10$ for all values of $n$ up to 25 . Hence we are able to write the higher moments of the largest exponential order statistic as

$$
E\left(X_{(n)}^{m}\right)=m ! S(n, n, 10)
$$

for $m$ greater than ten. The values of $S(n, n, 10)$ are in Table 3 for sample sizes of five to 25 . Hence $E\left(X_{(5)}^{20}\right)$ in a sample of size five is given by 20 ! (2.7661).

Table 3.

Values of $S(n, n, 10)$

\begin{tabular}{cccccc}
\hline $\mathrm{n}$ & $\mathrm{S}$ & $\mathrm{n}$ & $\mathrm{s}$ & $\mathrm{n}$ & $\mathrm{s}$ \\
\hline 5 & 2.7661 & 12 & 3.9668 & 19 & 4.6185 \\
6 & 3.0096 & 13 & 4.0796 & 20 & 4.6918 \\
7 & 3.2188 & 14 & 4.1843 & 21 & 4.7616 \\
8 & 3.4019 & 15 & 4.2820 & 22 & 4.8283 \\
9 & 3.5648 & 16 & 4.3737 & 23 & 4.8920 \\
10 & 3.7114 & 17 & 4.4599 & 24 & 4.9531 \\
11 & 3.8446 & 18 & 4.5413 & 25 & 5.0018 \\
\hline
\end{tabular}


CHAPTER III. ASYMPTOTIC MOMENTS FOR THE WEIBULL

Recall the transformation $X=Y^{1 / a}$ which takes the Welbull varlate, $Y$, with shape parameter a and scale parameter $b$ into the exponential with scale parameter $b$. In this chapter we will investigate the asymptotic properties of the exponential order statistics and use the results to obtain the asymptotic moments of the order statistics of the Weibuli.

Let the index $k$ be associated with the order statistic $X_{(n-k+1)}$ of a sample of size $n$ drawn from the distribution $F(x)$ which has $f(x)$ as the density function. Gumbel (16) defines $u_{k}$ to be the $k$-th characteristic

$$
u_{k}=F^{-1}\left(1-\frac{k}{n}\right)
$$

that 1s, $u_{k}$ is the value such that $P\left(X<u_{k}\right)=1-\frac{k}{n}$. Hence $u_{k}$ is the $100(n-k) / n$ percentage point of the distribution. Furthermore call $\mathrm{m}(\mathrm{x})$ the intensity function and $v_{k}$ the extremal intensity where

$$
m(x)=f(x) /(1-F(x))
$$

and

$$
v_{k}=\frac{n}{k} f\left(u_{k}\right)
$$

Finally call the random varlable $w_{k}$ the $k$-th reduced extreme where 


$$
w_{k}=v_{k}\left(x(n-k+1)^{\left.-u_{k}\right)}\right.
$$

where the $x^{\prime} s$ are the order statistics. The reader should note that the first reduced extreme is associated with the largest order statistic. For the exponential distribution with parameter $b, F(x)=1-e^{-x / b}$ and $f(x)=b^{-1} e^{-x / b}$. Hence $F^{-1}(y)=-b \log (1-y)$ and

$$
\begin{aligned}
u_{k} & =-b \log (1-(1-k / n)) \\
& =b \log (n / k) \\
m(x) & =\frac{b^{-1} e^{-x / b}}{1-\left(1-e^{-x / b}\right)} \\
& =b^{-1} \\
v_{k} & \frac{n}{k^{(}}\left(b^{-1} e^{-b^{-1}}(b \log (n / k))\right. \\
& =\frac{n}{k} b^{-1} e^{-10 g}(n / k) \\
& =b^{-1} \frac{n}{k} \frac{k}{n} \\
& =b^{-1} \\
& =b^{-1}(x(n-k+1)-b \log (n / k)) \\
k & b^{-1}(n-k+1)-\log (n / k) \\
& =b
\end{aligned}
$$

where log is the natural logarithm.

Fisher and Tippett (17) introduced three types of 
asymptotic distributions for the largest observation of a sample. Gumbel, in (37), quotes from an article written In French by the Russian probabilitist B. V. Gnedenko in which Gnedenko showed that these are the only posisible limiting forms and gave necessary and sufficient conditions for memberships in each class based on the initial distribution of the random variable $x$.

One of these limiting forms is the extreme value distribution

$$
F(z)=\exp (-\exp (-z))
$$

The condition for this limiting form of the largest observation is that

$$
\operatorname{Limit}_{n \rightarrow \infty} n P\left(x>u_{1}+x / v_{1}\right)=\exp (-x)
$$

Equation 3.7 holds for all $n$ for the exponential since

$$
u_{1}=b \log (n)
$$

and since $P(X>r)=1-F(r)$ we have

$$
\begin{aligned}
\underset{n \rightarrow \infty}{\operatorname{Limit}} & n P(x>b \log n+b x) \\
& =\underset{n \rightarrow \infty}{\operatorname{Limit}} n\left(\exp \left(-\frac{1}{b}(b \log n+b x)\right)\right) \\
& =\operatorname{Limit}_{n \rightarrow \infty} n(\exp (-\log n) \exp (-x))
\end{aligned}
$$




$$
\begin{aligned}
& =\operatorname{Limit} \mathrm{n}\left(\frac{1}{\mathrm{n}} \exp (-\mathrm{x})\right) \\
& =\exp (-x) .
\end{aligned}
$$

Fisher and Tippett (17, p. 183) gave another criterion which leads to the limiting form of Equation 3.6 .

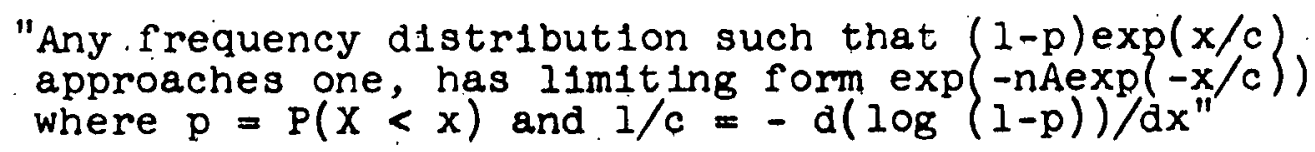

For the exponential distribution

$$
\begin{aligned}
1 / c & =-\frac{d}{d x}\left(\log \left(1-\left(1-e^{-x / b}\right)\right)\right) \\
& =-\frac{d}{d x}\left(\log \left(e^{-x / b}\right)\right) \\
& =-(-1 / b) \\
& =1 / 0 .
\end{aligned}
$$

Hence $c=b$ and

$$
(1-p) \exp (x / b)=\exp (-x / b) \exp (x / b)=1
$$

then the conduction is exact for all values of $n$ for the exponential distribution. The limiting form is then $\exp (-n \exp (-x / b))$. A third condition, given by Dodd (7), is that if $F(x)$ is a continuous distribution function such that $F(x)$ is less than one for all finite values of $x$, has two derivatives, and if $\lim _{n \rightarrow \infty} \frac{d}{d x}(1 / m(x))=0$, then $\operatorname{Limlt}_{n \rightarrow \infty}$ $P\left(v_{1}\left(x_{(n)}-u_{1}\right)-w\right)=\exp (-\exp (-w))$ for all real numbers $w$. 
The quantities $\mathrm{m}(\mathrm{x}), \mathrm{v}_{1}$, and $u_{1}$ are defined by Equations 3.1 . 3.2, and 3.3. Again the exponential distribution meets the condition exactly. The asymptotic form we will work with is then

$$
F\left(w_{I}\right)=\exp \left(-\exp \left(-w_{1}\right)\right)
$$

where $w_{1}$ is the first reduced extreme as defined in Equation 3.4. The asymptotic density is given by

$$
f\left(w_{1}\right)=\exp \left(-w_{1}\right) \exp \left(-\exp \left(-w_{1}\right)\right)
$$

We substitute for $w_{1}$ using Equation 3.5 to arrive at the asymptotic distribution in terms of the exponential variates, for the largest observation

$$
F(x(n))=\exp (-n \exp (-x / b))
$$

and the density function

$$
f(x(n))=n b^{-1} \exp (-x / b) \exp (-n \exp (-x / b))
$$

A. Asymptot1c Moment Generating Function for the Largest Observation

The moment generating function is derived in the usual manner. In terms of the reduced extreme

$$
G_{W_{1}}(t)=\int_{-\infty}^{\infty} \exp (t w) \exp (-w) \exp (-\exp (-w)) d w
$$

which, through the transformation $z=\exp (-w)$, leads to 


$$
\int_{0}^{\infty} z^{-t} \exp (-z) d z=\int_{0}^{\infty} z^{(1-t)-1} \exp (-z) d z
$$

which is a gamma function. The moment generating function is then

$$
G_{w_{1}}(t)=\Gamma(1-t)
$$

From Equation 3.5 we make the transformation from the reduced extreme to that of the largest order statistic of the exponential distribution. It is known that if a random variable. $Z$ has moment generating function $G_{Z}(t)$. then the random varlable $\mathrm{Y}=\mathrm{pZ}+\mathrm{q}$ has large sample moment generating function $a_{Y}(t)=\exp (q t) G_{Z}(p t)$. Hence

$$
G_{X(n)}(t)=\exp (b t \log n) \Gamma(1-b t) .
$$

We are able to get the asymptotic moments by the differentiation of Equation 3.13. It is necessary to take the derivatives of the gamma function. It is known that

$$
\Gamma(m)(x)=\int_{0}^{\infty} \exp (-z) z^{x-1}(\log z)^{m} d z
$$

where $\Gamma^{(m)}(x)$ is the $m$-th derivative of the gamma function. For our situation Equation 3.14 becomes

$$
\Gamma(m)(1-b t)=(-b)^{m} \cdot \int_{0}^{\infty} \exp (-z) z^{-b t}(\log z)^{m} d z
$$

This occurs since

$$
r(1-b t)=\int_{0}^{\infty} \exp (-z) \exp (-b t \log z) d z
$$




$$
\begin{aligned}
\frac{d}{d t} \Gamma(1-b t) & =\int_{0}^{\infty} \frac{d}{d t} \exp (-z) \exp (-b t \log z) d z \\
& =\int_{0}^{\infty} \exp (-z)(-b \log z) \exp (-b t \log z) d z .
\end{aligned}
$$

Hence

$$
\Gamma(1)(1-b t)=(-b) \int_{0}^{\infty} \exp (-z) \exp (-b t \log z) \log z d z .
$$

Now taking the second derivative

$$
\begin{aligned}
& \frac{d}{d t} \Gamma^{(1)}(1-b t)= \\
& (-b) \int_{0}^{\infty} \exp (-z)(-b \log z) \exp (-b t \log z) \log z d z \\
& \Gamma^{(2)}(1-b t)=(-b)^{2} \int_{0}^{\infty} \exp (-z) \exp (-b t \log z)(\log z)^{2} d z
\end{aligned}
$$

and the result of Equation 3.15 can be shown by mathematical induction. Now Equation 3.15 is, in itself, difficult to evaluate. However, for the moments, the derivative is evaluated at $t$ equal to zero and we have $\Gamma^{(m)}(1)$ where

$$
\Gamma(m)(1)=(-b)^{m} \int_{0}^{\infty} \exp (-z)(\log z)^{m} d z
$$

The integral of Equation 3.16 can be evaluated by using psi rurictions. From $(4 /)$ the psi function is $v(z)=\Gamma^{(1)}(z) /$ $\Gamma(z)$. Hence $\Gamma^{(1)}(z)=\phi(z) \Gamma(z)$. Taking derivatives and setting $z$ equal to one, we have

$$
\Gamma^{(m+1)}(1)=\sum_{1=0}^{m}\left(\begin{array}{c}
m \\
1
\end{array}\right) \Gamma^{(1)}(1) \downarrow^{(m-1)}(1)
$$


Now $(n)(1)=(-1)^{n+1} n ! z(n+1)$, where $z(s)=\sum_{k=1}^{\infty} k^{-s}$ is the Rlemann zeta function. The gamma, psi, and zeta functions are all tabulated in (44). A partial table of values of the integral is found in Table 4 . In the table the notation En represents the $n$-th power of ten.

Note that $\Gamma^{(0)}(1)$ is one. Furthermore, notice that the sign of $(-b)^{\mathrm{m}}$ is always the same as the sign of the Integral so that Equation 3.16 represents a positive quantity for all values of $\mathrm{m}$.

\begin{tabular}{|c|c|c|c|}
\hline $\mathrm{m}$ & $I(m)$ & $\mathrm{m}$. & $I(m)$ \\
\hline $\begin{array}{l}7 \\
2 \\
3 \\
4 \\
5\end{array}$ & $\begin{array}{l}.577216 \mathrm{EO} \\
.197811 \mathrm{E} 1 \\
.544487 \mathrm{E} 1 \\
.235615 \mathrm{E} 2 \\
.117839 \mathrm{E}\end{array}$ & $\begin{array}{r}6 \\
7 \\
8 \\
9 \\
10\end{array}$ & $\begin{array}{l}.715067 \mathrm{E} 3 \\
.501985 \mathrm{E} \\
.402436 \mathrm{E} \\
.362526 \mathrm{E} 6 \\
.362704 \mathrm{E} 7\end{array}$ \\
\hline
\end{tabular}

B. Generating Functions for Lower Order Statistics Now recall the relation of Equation 2.2 which we shall write as

$$
\begin{aligned}
& z_{n-k+1}=k\left(x_{(n-k+1)}-x_{(n-k)}\right) \\
& k=1,2, \ldots, n
\end{aligned}
$$


Now write Equation 3.17 for $k$ equal to one

$$
x_{(n)}=x_{(n-1)}+z_{n}
$$

Since $x_{(n-1)}$ and $z_{n}$ are independent we are able to write the moment generating function of the largest observation as

$$
G_{X(n)}(t)=G_{X(n-1)+z_{n}}(t)=G_{X_{(n-1)}}(t) G_{z_{n}}(t) .
$$

Since $z_{n}$ is an exponential varlate it has moment generating function

$$
G_{Z_{n}}(t)=(1-b t)^{-1}
$$

and Equation 3.19 becomes

$$
G_{X(n)}(t)=G_{X(n-1)}(t)(1-b t)^{-1}
$$

Multiplying Equation 3.21 by (1-bt) gives us the moment generuting function for $x_{(n-i)}$ in terms of the moment generating function of $x_{(n)}$.

$$
G_{X(n-1)}^{(t)}=G_{X_{(n)}}(t)(1-b t) .
$$

From Equation 3.13 for $G_{X_{(n)}}(t)$ we have

$$
G_{x(n-1)}(t)=\exp (b t \log n) \Gamma(1-b t)(1-b t) \text {. }
$$

The method above 1 easily extended, for $k$ small with respect 
to $n$, to obtain the asymptotic moment generating function for $x_{(n-k)}$ as

$$
a_{(n-k)}(t)=\exp (b t \log n) \Gamma(1-b t) j_{j=1}^{k}\left(1-\frac{b}{j} t\right)
$$

Taking derivatives we have

$$
\begin{aligned}
& G_{X}^{(m)}(t)=\sum_{1=0}^{m}\left(\begin{array}{c}
m \\
1
\end{array}\right) a_{X}^{(m-1)}(t) h(1, k, t) \\
& k=1,2, \ldots
\end{aligned}
$$

where

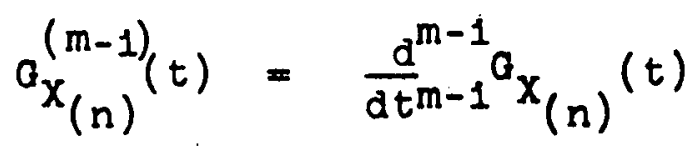

and

$$
n(1, k, t)=\frac{d^{1}}{d t^{1}} \prod_{j=1}^{k}\left(1-\frac{b}{j} t\right)
$$

\section{Asymptotic Moments}

From Equation 3.13 we find the approximate asymptotic moments of the largest observation as

$$
E^{*}\left(X_{(n)}^{m}\right)-b^{m} \sum_{1=0}^{m}(1)(\log n)^{m-1} I(1)
$$

where $I(1)$ are the absolute values of the integral in Table 4 with $I(0)=1$. The notation $E^{*}(X)$ wlll be used to denote the asymptotic expectation of $\mathrm{X}$.

To find the asymptotic moments of lower order stat1st1cs 
we use Equation 3.25 evaluated at $t$ equal to zero. Letting $b$ equal one we have

$E^{*}\left(X_{(n-k)}^{m}\right)=\sum_{1=0}^{m}\left(\begin{array}{l}m \\ 1\end{array}\right) \quad h(1, k, 0) E^{*}\left(X_{(n)}^{m-1}\right)$.

There is some difficulty in evaluating $h(1, k, 0)$.

Note that

$$
\prod_{j=1}^{k}(1-t / j)=(1-t)(1-t / 2)(1-t / 3) \ldots(1-t / k)
$$

whlch is a polynomial of degree $k$ in the varlable $t$. Hence there are at most $k$ nonzero derivatives of $\underset{i=1}{k}(1-t / j)$, and $h(1, k, 0)=0$ for $1>k$. Individual values may be found recursively using

$$
h(1, k+1,0)=-\frac{1}{k+I} h(1-1, k, 0)-h(1, k, 0)
$$

$$
1=1,2, \ldots \quad k=1,2, \ldots
$$

since $\prod_{j=1}^{k+1}(1-t / \jmath)=\left(1-\frac{t}{k+1}\right)_{j=1}^{k}(1-t / \jmath)$. Several values appear in Table 5 .

If the condition that $b=1$ is relaxed the right side of Equation 3.28 must be multiplied by $b^{m}$. 
Table 5.

Values of $h(1, k, 0)$

\begin{tabular}{rrrrrrrr}
\hline & 1 & 0 & 1 & 2 & 3 & 4 & 5 \\
\hline & 1 & 1 & -1 & 0 & 0 & 0 & 0 \\
2 & 1 & $-3 / 2$ & 1 & 0 & 0 & 0 \\
3 & 1 & $-11 / 6$ & $12 / 6$ & -1 & 0 & 0 \\
4 & 1 & $-25 / 12$ & $35 / 12$ & $-30 / 12$ & 1 & 0 \\
\hline
\end{tabular}

D. Asymptotic Crossproducts

In Chapter II we used the relation $x_{(k-j)}+w_{k, j}=$ $x_{(k)}$, where $x$ is an exponential random vartable and $w_{k, j}=$ $w_{k, j-1}+z_{k-j+1} /(n-k+j)$, to find the moments of the crossproducts of two exponential order statistics. The $z_{1}$ are the exponential variates defined in Equation 2.2. The moments of the $w_{k, j}$ are given by the recursive relation

$$
E\left(w_{k, j}^{m}\right)=m ! \sum_{1=0}^{m} E\left(w_{k, j-1}^{m}\right)\left(\frac{1}{n-k+j}\right)^{m-1 / 1 !}
$$

Hence the asymptotic moments of the crossproducts are

$$
\begin{aligned}
\left.E^{*}\left((x(k-j))^{X}(k)\right)^{m}\right) & =E^{*}\left(\left(x_{(k-j)}\left(x_{(k-j)}+w_{k, j}\right)\right)^{m}\right) \\
& =E^{*}\left(\left(x_{(k-j)}^{2}+x_{(k-j)} w_{k, j}\right)^{m}\right) \\
& =\sum_{1=0}^{m}\left(m_{1}^{m}\right) E^{*}\left(x_{(k-j)}^{m+1}\right) E\left(w_{k, j}^{m-1}\right)
\end{aligned}
$$


Then the approximate asymptot1c covariances are given by

$$
C^{*}\left(x_{(k)}^{m}, x_{(j)}^{m}\right)=E^{*}\left(\left(X_{(k)} x_{(j)}\right)^{m}\right)-E^{*}\left(X_{(k)}^{m}\right) E^{*}\left(X_{(j)}^{m}\right)
$$

where $C^{*}$ denotes the asymptotic covariance. 
CHAPTER IV. THE EXPONENTIAL DISTRIBUTION

The exponential distribution is a Weibuli distribution with a shape parameter of one. The exponential density is given by

$$
\begin{aligned}
g(x ; b) & =b^{-I} \exp (-x / b) \quad x>0, b>0 \\
& =0 \text { otherwise. }
\end{aligned}
$$

Purthermore

$$
E(x)=b, \quad V(x)=b^{2}
$$

From Equation 2.7, the expected value of the $k$-th order statistic is

$$
E(x(k))=b S(n, k, 1)
$$

and has as its variance

$$
v\left(x(k)=b^{2}\left(s(n, k, 2)-s^{2}(n, k, 1)\right) .\right.
$$

Sarhan (36) has written these relations as

$$
E(X(k))=b \sum_{j=1}^{k} 1 /(n-j+1)
$$

and

$$
v\left(x_{(k)}\right)=b^{2} \sum_{j=1}^{k} i /(n-j+1)^{2}
$$

Furthermore, if $k$ is less than 1 , the covariance is 


$$
C\left(X_{(k)}, X_{(1)}\right)=V\left(X_{(k)}\right)
$$

The values for Equations 4.5 and 4.7 may be found in the first columns of Table 1 and Table 2 .

Considering the asymptotic case we have from Equation 3.27, setting $m$ equal to one

$$
E^{*}(X(n))=b(\log n+I(1))=b(\log n+.5772)
$$

This is not surprising since it is known that $\int_{1}^{n} \frac{d t}{t}=$ $\log \mathrm{n}$. Hence $\log \mathrm{n}$ approximates the sum of the reciprocals $\sum 1 / t$. Values of Equation 4.8 for $b=1$ are compared with the finite form of Equation 4.3 with $k=n$, in Table 6 . The asymptotic formia is a close, simple, approximation to the exact value. The error is about 4 percent at $n=5$ and decreases to about one percent for $n=100$.

To compute the lower asymptotic expectations, we use Equation 3.28

$$
\begin{aligned}
E^{*}\left(x_{(n-k)}\right) & =E^{*}(X(n))-b \sum_{j=1}^{k} 1 / j \\
& =b\left(10 g n+I(1)-\sum_{j=1}^{k} 1 / j\right) \\
k & =1,2, \ldots, n-1
\end{aligned}
$$

The error of the approximation is a constant and equal to the error in the approximation to the largest order 
Table 6.

Comparison of the finite and asymptotic forms of the expected value of the largest exponential order statistic

\begin{tabular}{rccccc}
\hline$n$ & $E^{*}\left(X_{(n)}\right)$ & $E^{*}\left(X_{(n)}\right)-E(X(n))$ & $n$ & $E^{*}(X(n))$ & $E^{*}(X(n))-E(X(n)$ \\
\hline 5 & 2.3800 & .0967 & 55 & 4.5845 & .0087 \\
10 & 2.9783 & .0493 & 60 & 4.6715 & .0083 \\
15 & 3.3512 & .0330 & 65 & 4.7516 & .0076 \\
20 & 3.6225 & .0248 & 70 & 4.8257 & .0071 \\
25 & 3.8358 & .0198 & 75 & 4.8947 & .0066 \\
30 & 3.9784 & .0165 & 80 & 4.9592 & .0062 \\
35 & 4.1275 & .0142 & 85 & 5.0198 & .0058 \\
40 & 4.2661 & .0124 & 90 & 5.0770 & .0055 \\
45 & 4.3838 & .0110 & 95 & 5.1311 & .0052 \\
50 & 4.4892 & .0099 & 100 & 5.1824 & .0049 \\
\hline
\end{tabular}


statistic. From Equation 3.27 and setting b equal to one we have the second asymptotic moment of the largest order statistic of an exponential distribution as

$$
\begin{aligned}
E^{*}\left(X_{(n)}^{2}\right) & =\log ^{2} n+2 I(1) \log n+I(2) \\
& =\log ^{2} n+1.1544 \log n+1.9781 .
\end{aligned}
$$

The I(1) are from Table 4. Equations 4.8 and 4.10 give us the asymptotic variance of the largest observation.

$$
\begin{aligned}
V^{*}\left(X_{(n)}\right)= & E^{*}\left(X_{(n)}^{2}\right)-E^{* 2}(X(n)) \\
= & \left(\log ^{2} n+2 I(1) \log n+I(2)\right)-\left(\log ^{2} n\right. \\
& \left.\quad+2 I(1) \log n+(I(I))^{2}\right) \\
= & I(2)-(I(1))^{2} .
\end{aligned}
$$

F rom (44), it is known that $I(2)-(I(1))^{2}=\pi^{2} / 6$. Hence Fquation 4.11 is independent of semple size. We note that from Equation 4.6

$$
v(x(k))=\sum_{j=1}^{k} 1 /(n-j+1)^{2}
$$

which can be written for $k=n$ as

$$
v\left(x_{(n)}\right)=\sum_{j=1}^{n} 1 / j^{2}
$$

Now if we let $n$ increase without bound it. is known that 


$$
\operatorname{Limit}_{n \rightarrow \infty} \sum_{j=1}^{n} 1 / j^{2}=\pi^{2} / 6
$$

which is exactiy the same as Equation 4.11 . If the asymptotic value is compared to the values in Table 2 we see that the error of approximation is only about two percent at $n$ equal to 25 .

From Equation 3.28 we have

$$
E^{*}\left(X_{(n-1)}\right)=E^{*}\left(X_{(n)}\right)-1
$$

and

$$
E^{*}\left(x_{(n-1)}^{2}\right)=E^{*}\left(x_{(n)}^{2}\right)-2 E^{*}(x(n))
$$

hence

$$
\begin{aligned}
V^{*}\left(X_{(n-1)}\right) & =E^{*}\left(X_{(n-1)}^{2}\right)-E^{* 2}\left(X_{(n)}\right) \\
& =\left[E^{*}\left(X_{(n)}^{2}\right)-2 E^{*}\left(X_{(n)}\right)\right]-\left[E^{*}\left(X_{(n)}\right)-1\right]^{2} \\
& =E^{*}\left(X_{(n)}^{2}\right)-E^{* 2}\left(X_{(n)}\right)-1 \\
V^{*}\left(X_{(n-1)}\right) & =V^{*}\left(X_{(n)}\right)-1
\end{aligned}
$$

Continuing, this procedure we find that

$$
\begin{aligned}
V^{*}(x(n-k)) & =V^{*}(x(n))-\sum_{j=1}^{k} 1 / j^{2} \\
& =\frac{\pi^{2}}{\sigma}-\sum_{j=1}^{k} 1 / j^{2}
\end{aligned}
$$

which is exactly the value we would expect based on Equation 
4.6. The use of the asymptotic expression as an approximation for the variance of an exponential order statistic has a constant error over the whole sample and that error is

$$
\begin{aligned}
V^{*}(X(n))-V(X(n)) & =\frac{\pi^{2}}{6}-\sum_{1=1}^{n} 1 / 1^{2} \\
& =\sum_{1=n+1}^{\infty} 1 / 1^{2} .
\end{aligned}
$$

Hence the percent error for the largest statistic is $\left(100 \sum_{1=n+1}^{\infty} 1 / 1^{2}\right) /\left(\pi^{2} / 6\right)$. The asymptotic forms developed above for expectations and variances give us close approximations for values outside the range of Tables 1 and 2 . These results give us a quick test value based on the larger asymptotic variances. Furthermore, it is interesting that the theory based on the asymptotic properties for the largest order statistic fits so well over the whole sample value range.

A. Censored Estimation of the Mean

We shall next consider a special class of estimators of the mean, $b$, of an exponential distribution. Consider the random variables $z_{r}$ defined in Equation 1.6 . Since the $z_{r}$ are independently and identically distributed as exponential with parameter $b$, their characteristic function is given by

$$
g_{z_{r}}(t)=(1-1 t b)^{-1}
$$


Consider a sum of the $z_{r}$. It is seen that

$$
\sum_{j=1}^{n-r} z_{j}=x_{(1)}+x_{(2)} \cdots+x_{(n-r-1)}+r x_{(n-r)}
$$

Let $z_{k}=(n-k+1)(x(k)-x(k-1))$ be the observed values of $z_{k}$ and

$$
\hat{w}=\sum_{j=1}^{n-r} z_{j} / D
$$

where $D$ is a suitably chosen constant denominator. The characteristic function of $\hat{w}$ is then

$$
B_{\hat{w}}(t)=(1-1 t b / D)^{-(n-r)}
$$

so that its expectation is

$$
E(\hat{w})=(n-r) b / D \text {. }
$$

Let $h=\hat{w}-b$. The random variable $h$ has as 1 ts characteristic function

$$
g_{h}(t)=\exp (-1 t b)(1-1 t b / D)^{-(n-r)}
$$

and expectation

$$
E(h)=b\left(\frac{(n-r)-D}{D}\right)
$$

The right-hand side of Equation 4.22 is exactly the bias of the statistic $\hat{w}$ for estimating $b$. Furthermore, the second derivative of Equation 4.21 gives the mean square (MSE) of $\hat{w}$ for estimating $b$. 
60

$$
\begin{aligned}
\operatorname{MSE}(\hat{w}) & =b^{2}\left(i-2((n-r) / D)+\left((n-r) / D^{2}\right)\right. \\
& \left.+((n-r) / D)^{2}\right)
\end{aligned}
$$

Consider the following special cases of the estimator $\hat{w}$.

$$
\begin{aligned}
& \bar{x}=\sum_{j=1}^{n} z_{j} / n \\
& \hat{E}_{r}=\sum_{j=1}^{n-r} z_{j} /(n-r) \\
& \hat{w}_{r}=\underset{j=1}{n-r} z_{j} / n \\
& \hat{M}_{r}=\sum_{j=1}^{n-r} z_{j} /(n-r+1)
\end{aligned}
$$

Equation 4.24 is the usual mean estimator. $\hat{E}_{r}$ is the est1mator discussed earller which was developed by Epstein and $\hat{w}_{r}$ is the Winsorized mean. The estimator $\hat{M}_{r}$ is developed by minimizing Equation 4.23 with respect to $D$ and is thus the minimum MSE of this class of estimators for the exponential. The expected values of these estimators are

$$
\begin{aligned}
& E(\bar{X})=b \\
& E\left(\hat{E}_{r}\right)=b \\
& E\left(\hat{W}_{r}\right)=b(1-r / n) \\
& E\left(\hat{M}_{r}\right)=b(1-(1 /(n-r+1)) .
\end{aligned}
$$

Note that $\bar{X}$ and $\hat{E}_{r}$ are unblased and all of the estimators are consistent for fixed $r$. The MSE of the estimates are 


$$
\begin{aligned}
& \operatorname{MSE}(\bar{X})=b^{2} / n \\
& \operatorname{MSE}\left(\hat{E}_{r}\right)=\left(b^{2} / n\right)(1+r /(n-r)) \\
& \operatorname{MSE}\left(\hat{W}_{r}\right)=\left(b^{2} / n\right)(1+r(r-1) / n) \\
& \left.\operatorname{MSE}\left(\hat{M}_{r}\right)=\left(b^{2} / n\right)(n-(n-r) /(n-r+1))\right)
\end{aligned}
$$

If we define relative efficiency with respect to mean of the estimators as $\operatorname{MSE}(\bar{X}) / \operatorname{MSE}(\hat{W})$ and denote this by REM, then the REM of the three estimators are given by

$$
\begin{aligned}
& \operatorname{REM}\left(\hat{E}_{r}\right)=(n-r) / n \\
& \operatorname{REM}\left(\hat{w}_{r}\right)=n /(n+r(r-1)) \\
& \operatorname{REM}\left(\hat{M}_{r}\right)=(n-r+1) / n
\end{aligned}
$$

Note that in the case of the exponential, the maximum likelihood estimate for $b$ is $\bar{X}$. The values of REM will be tabulated for the Welbull distribution in the next chapter. 'We note that $\hat{M}_{O}$ is the minimum mean square error estimate for the parameter of the exponentlal and $\operatorname{REM}\left(\hat{M}_{0}\right)=(n+1) / n$ whlch is greater than one and therefore more efficient then the usual mean. 
CHAPTER V. ESTIMATION AND TESTING IN THE

\section{WEIBULL DISTRIBUTION}

For convenience, we have chosen to write the Weibull distribution in this paper as

$$
\begin{aligned}
f(y ; a, b) & =\frac{1}{a b} y^{\frac{1}{a}-1} \exp \left(-y^{1 / a / b}\right) \quad y>0, a>0, b>0 \\
& =0 \text { otherwise. }
\end{aligned}
$$

A. Maximum Likelihood Estimation for the Mean

In Chapter I the maximum likelihood estimates (MLE) for the parameters of the Welbull distribution were developed. From Equation 1.12 the MLE are the solutions of

$$
\begin{aligned}
& \hat{b}=\frac{1}{n} \Sigma y^{1 / \hat{a}} \\
& -\hat{a}+\frac{1}{n \hat{b}} \sum y^{1 / \hat{a}} \log y=\frac{1}{n} \sum \log y
\end{aligned}
$$

Furthermore the mean of the Weibull as derived in Equation 1.10 is

$$
E(Y)=m=c \Gamma(a+1)
$$

where $b^{a}=c$. Hence the maximum likelihood estimate of $m$ is

$$
\hat{m}=\hat{c} \Gamma(\hat{a}+1)
$$

where $\hat{c}=\left(\frac{1}{n} \sum y^{1 / \hat{a}}\right)^{\hat{a}} \cdot$ By a two parameter Taylor series expansion 


$$
\hat{m}-m \doteq \Gamma(a+1)(\hat{c}-c)+c \Gamma^{(1)}(a+1)(\hat{a}-a) \text {. }
$$

It is known that the MLE of the Welbull distribution are cons1stent, asymptotically efflcient, and asymptotically normal Hence

$$
E^{*}(\hat{m}-m)=0
$$

and

$$
\begin{aligned}
V^{*}(\hat{m}) & \doteq(\Gamma(a+1))^{2} V^{*}(\hat{c})+c^{2}\left(\Gamma^{(1)}(a+1)\right)^{2} V^{*}(\hat{a}) \\
& +2 c \Gamma(a+1) \Gamma^{(1)}(a+1) c^{*}(\hat{a}, \hat{c})
\end{aligned}
$$

where $E^{*}, V^{*}$, and $C^{*}$ represent the asymptotic expected values, varlances, and covariances. It is known that the limiting covariance matrix of the MLE of the parameters $c$ and a is given by

$$
\frac{1}{n}\left(\begin{array}{cc}
1.1087(c a)^{2} & -.2570 c a^{2} \\
-.2570 c a^{2} & .6079 a^{2}
\end{array}\right)
$$

Suhatituting into Equation 5.7 . We have

$$
\begin{aligned}
& n V^{*}(\hat{m}) \doteq 1.1087(\Gamma(a+1) c a)^{2}+.6079\left(\Gamma^{(1)}(a+1) c a\right)^{2} \\
& -.5140 r(a+1) r^{(1)}(a+1) c^{2} a^{2}
\end{aligned}
$$

From Equation 1.10 we have

$$
\begin{aligned}
& E(Y)=c \Gamma(a+1) \\
& E\left(Y^{2}\right)=c^{2} \Gamma(2 a+1)
\end{aligned}
$$

The derivation of this result is given in the Appendix. 
and therefore

$$
V(Y)=c^{2}\left(\Gamma(2 a+1)-(\Gamma(a+1))^{2}\right) .
$$

Then from Equations 5.10 and 5.11 the mean square error (MSE) of the estimate of the mean $\bar{y}=\frac{l}{n} r y_{1}$ is given by

$$
\operatorname{MSE}(\bar{y})=\frac{c^{2}}{n}\left(\Gamma(2 a+1)-(\Gamma(a+1))^{2}\right) \text {. }
$$

If we compare the approximate asymptotic variance of the MLE with the MSE of $\bar{y}$, we have an asymptotic relative efficiency.

$$
\frac{\mathrm{V}^{*}(\hat{\mathrm{m}})}{\operatorname{MSE}(\overline{\mathrm{y}})}=
$$

$$
\begin{gathered}
\frac{1.1087(\Gamma(a+1) a)^{2}+.6079\left(\Gamma^{(1)}(a+1) a\right)^{2}-.514 \Gamma(a+1) \Gamma^{(1)}(a+1) a^{2}}{\Gamma(2 a+1)-(\Gamma(a+1))^{2}} \\
=\frac{1.1087 a^{2}+.6079(1(a+1) a)^{2}-.514 *(a+1) a^{2}}{\left(\frac{\Gamma(2 a+1)}{\Gamma(a+1))^{2}-1}\right.}
\end{gathered}
$$

where $*(a+1)=\Gamma(1)(a+1) / \Gamma(a+1)$. Note that Equation 5.13 is independent of the scale parameter. In Table 7 we compare this efficiency for several values of the shape parameter. It is seen that as the shape parameter increases beyond one the relative efficlency decreases, that is the MLE becomes more efficient with respect to $\bar{y}$. Study of Equation 5.13 shows why this reduction occurs since $\Gamma(2 a+1)$ is clearly the dominate term as the shape parameter increases. Hence, for 
Table 7 .

Asymptotic relative efficiency $V^{*}(m) / \operatorname{MSE}(\bar{y})$

\begin{tabular}{lllllll} 
Shape parameter.5 & 1.0 & 2.0 & 3.0 & 4.0 & 5.0 \\
\hline Efflclency & .99795 & 1.0000 & .92240 & .68068 & .39884 & .17563
\end{tabular}

larger values of the shape parameter, the maximum likelihood estimator provides large gains in efflclency. The gains Increase from about 10 percent for $a=a$ to over 500 percent at $a=5$.

\section{B. Censored Est1mation}

In the previous chapter we studied the class of est1mators

$$
\hat{w}=(\stackrel{n-r}{\Sigma} y(1)+r y(n-r)) / D
$$

for the mean of the exponential distribution. We propose to now investigate this class of estimators for estimation of the mean of the Welbull distribution. We have defined in Chapter IV the relative efficiency (REM) of the class of estimators defined by $w$ as being the mean square error of the mean, $\bar{y}$, divided by the mean square error of $w$. For a given sample size $n$ let 


$$
A^{\prime}=(1,1, \ldots, 1, r+1,0, \ldots 0)
$$

be a lxn vector giving the linear combination of the order statistics of the estimator $\hat{w}$. Let $Y$ be the $n \times I$ vector of ordered observations. Then

$$
\hat{w}=A^{\prime} Y / D
$$

and the expectation is

$$
E(\hat{w})=b^{a} A \cdot \varepsilon / D
$$

where $\varepsilon$ is an $n \times 1$ vector of the expected values of the Welbull variates with scale parameter one. These values were computed in Chapter II for samples up to size ten. A table for samples up to 25 is found in (28). Now

$$
V\left(A^{\prime} I / D\right)=b^{2 a} A \cdot \$ A / D^{2}
$$

where $\$$ is an nxn matrix of covariances of the Weibull with unit scale parameter. A partial table of these values is found in Chapter II. Now the squared bias of the estimator is given by

$$
\operatorname{BIas}^{2}(\hat{w})=b^{2 a}(A \cdot \varepsilon-D a !)^{2} / D^{2}
$$

Hence the mean square error of $\hat{w}$ is

$$
\operatorname{MSE}(\hat{w})=\frac{b^{2 a}}{D^{2}}\left(A^{\prime} \ddagger A+\left(A^{\prime} \varepsilon-D a !\right)^{2}\right)
$$


Using Equation 5.12 the relative efficlency with respect to the mean is

$$
\operatorname{REM}(\hat{w})=\left(\frac{D^{2}}{n}\right) \frac{\left((2 a) d-(a b)^{2}\right)}{A+A+(A \cdot E-D a d)^{2}}
$$

Recall from Chapter IV that we have introduced three cases of the estimator $\hat{w} ; \hat{W}_{r}$, the winsorized mean; $\hat{E}_{r}$, the Epstein mean; and $\widehat{M}_{r}$, the minimum mean square estimator of this class for the exponential. The relative efficiencies for these estimators are given in Table 8 for samples of size two to 25 , shape parameters of one through five, and for the censoring indicated by $r$. For $r$ equal to zero the relative efficlencles are all equal to one.

From the empirical results of Table 8 , we see that for all amounts of censoring, the REMs for the exponential are less than those for the corresponding estimator for the Weibulls with shape parameter greater than one. More importantly, as the shape parameter increases, the efficlency. increases. Note however, that as censoring increases bejond two, the officlency decreases for a given shape parameter. The gains in efficiency are quite large for large shape parameters. For the once censored case in a sample of size 25, the gains range from 20 percent for $a=2$ up to 1000 percent at $a=5$. The largest gains in efflciency for the once censored samples occur when the sample size is small. 
Table 8.

REMs of $r$ of $n$ censored estimators of the mean of a Welbull with integer shape parameter

\begin{tabular}{|c|c|c|c|c|c|c|c|c|c|}
\hline $\begin{array}{c}\text { Shape } \\
n\end{array}$ & $\begin{array}{l}\mathrm{pa} \\
\mathbf{r}\end{array}$ & $\widehat{\mathrm{W}}_{r}$ & $\stackrel{7}{E}_{r}$ & $\widehat{\mathrm{M}}_{\mathrm{r}}$ & $\hat{\mathrm{w}}_{\mathbf{r}}$ & $\stackrel{R}{E}_{r}$ & $\hat{\mathrm{M}}_{\mathrm{r}}$ & $\hat{\mathrm{W}}_{\mathrm{r}}$ & $\frac{3}{E_{r}}$ \\
\hline $\begin{array}{l}2 \\
3 \\
4 \\
5 \\
5 \\
6 \\
6 \\
7 \\
7 \\
8 \\
8 \\
9 \\
9 \\
10 \\
10 \\
10 \\
11 \\
11 \\
11 \\
12 \\
12 \\
12 \\
13 \\
13 \\
13 \\
14 \\
14 \\
14 \\
15 \\
15 \\
15 \\
15 \\
16 \\
16 \\
16 \\
16 \\
17 \\
17 \\
17 \\
17 \\
18 \\
18\end{array}$ & 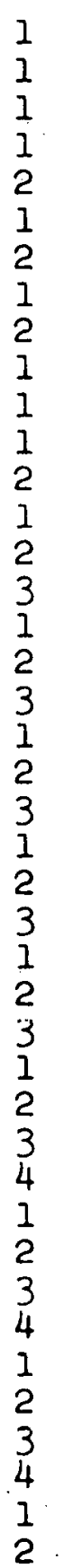 & $\begin{array}{l}1.00 \\
1.00 \\
1.00 \\
1.00 \\
0.71 \\
1.00 \\
0.75 \\
1.00 \\
0.78 \\
1.00 \\
0.80 \\
1.00 \\
0.82 \\
1.00 \\
0.83 \\
0.62 \\
1.00 \\
0.85 \\
0.65 \\
1.00 \\
0.86 \\
0.67 \\
1.00 \\
0.87 \\
0.68 \\
1.00 \\
0.87 \\
0.70 \\
1.00 \\
0.88 \\
0.71 \\
0.56 \\
1.00 \\
0.89 \\
0.73 \\
0.57 \\
1.00 \\
0.89 \\
0.74 \\
0.59 \\
1.00 \\
0.90\end{array}$ & $\begin{array}{l}0.50 \\
0.67 \\
0.75 \\
0.80 \\
0.60 \\
0.83 \\
0.67 \\
0.86 \\
0.71 \\
0.88 \\
0.75 \\
0.89 \\
0.78 \\
0.90 \\
0.80 \\
0.70 \\
0.91 \\
0.82 \\
0.73 \\
0.92 \\
0.83 \\
0.75 \\
0.92 \\
0.85 \\
0.77 \\
0.93 \\
0.86 \\
0.79 \\
0.93 \\
0.87 \\
0.80 \\
0.73 \\
0.94 \\
0.87 \\
0.81 \\
0.75 \\
0.94 \\
0.88 \\
0.82 \\
0.76 \\
0.94 \\
0.89\end{array}$ & $\begin{array}{l}1.00 \\
1.00 \\
1.00 \\
1.00 \\
0.80 \\
1.00 \\
0.83 \\
1.00 \\
0.86 \\
1.00 \\
0.88 \\
1.00 \\
0.89 \\
1.00 \\
0.90 \\
0.80 \\
1.00 \\
0.91 \\
0.82 \\
1.00 \\
0.92 \\
0.83 \\
1.00 \\
0.92 \\
0.85 \\
1.00 \\
0.93 \\
0.86 \\
1.00 \\
0.93 \\
0.87 \\
0.80 \\
1.00 \\
0.94 \\
0.87 \\
0.81 \\
1.00 \\
0.94 \\
0.88 \\
0.82 \\
1.00 \\
0.94\end{array}$ & $\begin{array}{l}2.86 \\
2.27 \\
1.99 \\
1.82 \\
1.58 \\
1.70 \\
1.49 \\
1.62 \\
1.42 \\
1.56 \\
1.37 \\
1.51 \\
1.34 \\
1.47 \\
1.31 \\
1.04 \\
1.44 \\
1.28 \\
1.02 \\
1.41 \\
1.26 \\
1.01 \\
1.38 \\
1.24 \\
1.00 \\
1.36 \\
1.23 \\
0.99 \\
1.34 \\
1.22 \\
0.98 \\
0.80 \\
1.33 \\
1.20 \\
0.98 \\
0.79 \\
1.31 \\
1.19 \\
0.97 \\
0.79 \\
1.30 \\
1.18\end{array}$ & $\begin{array}{l}1.67 \\
1.69 \\
1.63 \\
1.57 \\
1.61 \\
1.52 \\
1.55 \\
1.48 \\
1.50 \\
1.44 \\
1.46 \\
1.41 \\
1.43 \\
1.39 \\
1.40 \\
1.26 \\
1.37 \\
1.38 \\
1.24 \\
1.35 \\
1.35 \\
1.22 \\
1.33 \\
1.34 \\
1.21 \\
1.32 \\
1.32 \\
1.20 \\
1.30 \\
1.30 \\
1.18 \\
1.04 \\
1.29 \\
1.29 \\
1.17 \\
1.03 \\
1.28 \\
1.28 \\
1.16 \\
1.03 \\
1.27 \\
1.27\end{array}$ & $\begin{array}{l}2.86 \\
2.27 \\
1.99 \\
1.82 \\
1.64 \\
1.70 \\
1.55 \\
1.62 \\
1.49 \\
1.56 \\
1.44 \\
1.51 \\
1.40 \\
1.47 \\
1.37 \\
1.18 \\
1.44 \\
1.34 \\
1.17 \\
1.41 \\
1.32 \\
1.15 \\
1.38 \\
1.30 \\
1.14 \\
1.36 \\
1.28 \\
1.13 \\
1.34 \\
1.27 \\
1.12 \\
0.97 \\
1.33 \\
1.26 \\
1.11 \\
0.96 \\
1.31 \\
1.24 \\
1.10 \\
0.96 \\
1.30 \\
1.23\end{array}$ & $\begin{array}{l}8.94 \\
6.34 \\
5.13 \\
4.42 \\
4.50 \\
3.95 \\
3.98 \\
3.61 \\
3.61 \\
3.35 \\
3.34 \\
3.15 \\
3.12 \\
2.99 \\
2.95 \\
2.54 \\
2.85 \\
2.81 \\
2.41 \\
2.74 \\
2.69 \\
2.30 \\
2.64 \\
2.59 \\
2.21 \\
2.56 \\
2.50 \\
2.13 \\
2.49 \\
2.43 \\
2.06 \\
1.79 \\
2.42 \\
2.36 \\
2.00 \\
1.74 \\
2.36 \\
2.30 \\
1.95 \\
1.69 \\
2.31 \\
2.24\end{array}$ & $\begin{array}{l}5.43 \\
4.81 \\
4.25 \\
3.84 \\
4.54 \\
3.54 \\
4.07 \\
3.30 \\
3.72 \\
3.11 \\
3.45 \\
2.96 \\
3.24 \\
2.83 \\
3.07 \\
2.78 \\
2.72 \\
2.93 \\
2.64 \\
2.63 \\
2.81 \\
2.52 \\
2.54 \\
2.70 \\
2.42 \\
2.47 \\
2.61 \\
2.311 \\
2.41 \\
2.54 \\
2.26 \\
2.00 \\
2.35 \\
2.47 \\
2.20 \\
1.94 \\
2.30 \\
2.40 \\
2.114 \\
1.89 \\
2.25 \\
2.35\end{array}$ \\
\hline
\end{tabular}




\begin{tabular}{|c|c|c|c|c|c|c|}
\hline$\hat{\mathrm{M}}_{r}$ & $\hat{W}_{r}$ & $\widehat{t}_{\mathrm{r}}$ & $\hat{M}_{r}$ & $\hat{\mathrm{W}}_{\mathrm{r}}$ & ${ }_{\mathrm{r}}^{5}$ & $\hat{\mathrm{M}}_{\mathrm{r}}$ \\
\hline $\begin{array}{l}8.94 \\
6.34 \\
5.13 \\
4.42 \\
4.56 \\
3.95 \\
4.05 \\
3.61 \\
3.68 \\
3.35 \\
3.41 \\
3.15 \\
3.19 \\
2.99 \\
3.02 \\
2.68 \\
2.85 \\
2.87 \\
2.55 \\
2.74 \\
2.75 \\
2.44 \\
2.64 \\
2.65 \\
2.34 \\
2.56 \\
2.56 \\
2.26 \\
2.49 \\
2.48 \\
2.19 \\
1.94 \\
2.42 \\
2.41 \\
2.13 \\
1.88 \\
2.36 \\
2.35 \\
2.07 \\
1.82 \\
2.31 \\
2.30\end{array}$ & $\begin{array}{l}30.04 \\
20.12 \\
15.55 \\
12.89 \\
14.67 \\
11.15 \\
12.56 \\
9.91 \\
11.06 \\
8.99 \\
9.94 \\
8.27 \\
9.08 \\
7.69 \\
8.38 \\
7.70 \\
7.21 \\
7.82 \\
7.14 \\
6.81 \\
7.34 \\
6.68 \\
6.47 \\
6.94 \\
6.29 \\
6.17 \\
6.60 \\
5.95 \\
5.92 \\
6.30 \\
5.66 \\
5.23 \\
5.69 \\
6.03 \\
5.41 \\
4.98 \\
5.49 \\
5.80 \\
5.19 \\
4.76 \\
5.31 \\
5.59\end{array}$ & $\begin{array}{l}18.71 \\
15.43 \\
12.95 \\
11.23 \\
14.67 \\
9.99 \\
12.64 \\
9.05 \\
11.18 \\
8.32 \\
10.08 \\
7.74 \\
9.23 \\
7.25 \\
8.54 \\
7.98 \\
6.85 \\
7.97 \\
7.41 \\
6.50 \\
7.50 \\
6.94 \\
6.21 \\
7.09 \\
6.54 \\
5.95 \\
6.75 \\
6.20 \\
5.72 \\
6.44 \\
5.90 \\
5.45 \\
5.51 \\
6.18 \\
5.64 \\
5.20 \\
5.33 \\
5.94 \\
5.41 \\
4.97 \\
5.17 \\
5.73\end{array}$ & $\begin{array}{r}30.04 \\
20.12 \\
15.55 \\
12.89 \\
14.73 \\
11.15 \\
12.63 \\
9.91 \\
11.14 \\
8.99 \\
10.03 \\
8.27 \\
9.16 \\
7.69 \\
8.47 \\
7.87 \\
7.21 \\
7.90 \\
7.31 \\
6.81 \\
7.42 \\
6.84 \\
6.47 \\
7.02 \\
6.44 \\
6.17 \\
6.67 \\
6.11 \\
5.92 \\
6.37 \\
5.81 \\
5.38 \\
5.69 \\
6.11 \\
5.56 \\
5.13 \\
5.49 \\
5.87 \\
5.33 \\
4.91 \\
5.31 \\
5.66\end{array}$ & $\begin{array}{r}106.03 \\
68.97 \\
51.94 \\
42.12 \\
51.27 \\
35.71 \\
43.20 \\
31.19 \\
37.47 \\
27.82 \\
33.19 \\
25.21 \\
29.88 \\
23.12 \\
27.24 \\
26.09 \\
21.41 \\
25.08 \\
23.92 \\
19.99 \\
23.28 \\
22.12 \\
18.78 \\
21.76 \\
20.60 \\
17.74 \\
20.46 \\
19.30 \\
16.84 \\
19.33 \\
18.18 \\
17.48 \\
16.04 \\
18.34 \\
17.20 \\
16.49 \\
15.34 \\
17.47 \\
16.33 \\
15.62 \\
14.71 \\
16.69 \\
23\end{array}$ & $\begin{array}{l}67.50 \\
53.39 \\
43.48 \\
36.78 \\
51.14 \\
32.03 \\
43.23 \\
28.49 \\
37.59 \\
25.75 \\
33.36 \\
23.57 \\
30.07 \\
21.79 \\
27.24 \\
26.44 \\
20.31 \\
25.29 \\
24.27 \\
19.06 \\
23.49 \\
22.46 \\
17.98 \\
21.97 \\
20.93 \\
17.05 \\
20.67 \\
19.62 \\
16.24 \\
19.54 \\
18.49 \\
17.74 \\
15.51 \\
18.55 \\
17.50 \\
16.74 \\
14.87 \\
17.67 \\
16.63 \\
15.87 \\
14.29 \\
16.89\end{array}$ & 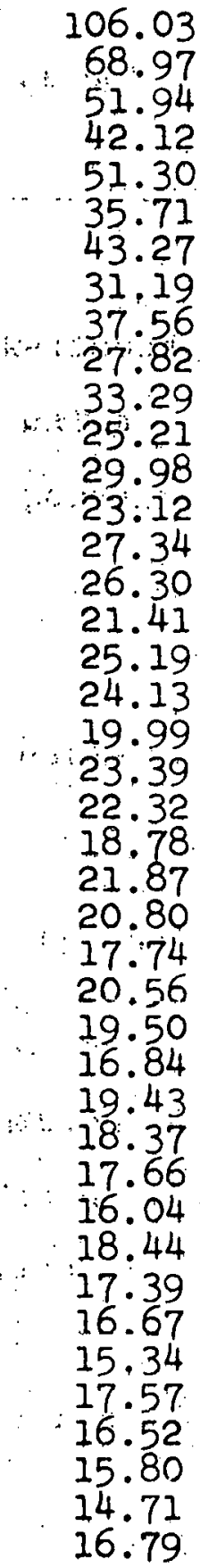 \\
\hline
\end{tabular}


Table 8. (Continued)

\begin{tabular}{|c|c|c|c|c|c|c|c|c|c|}
\hline $\begin{array}{c}\text { Shape } \\
\text { n }\end{array}$ & & $\begin{array}{l}\hat{W}_{r} \\
\hat{W}_{r}\end{array}$ & $\mathrm{E}_{\mathrm{r}}$ & $\hat{M}_{r}$ & $\hat{W}_{r}$ & $\stackrel{\mathcal{E}}{\mathrm{E}}_{\mathrm{r}}$ & $\hat{\mathrm{M}}_{\mathrm{r}}$ & $\hat{\mathrm{W}}_{\mathrm{r}}$ & $\hat{E}_{r}$ \\
\hline $\begin{array}{l}18 \\
18 \\
19 \\
19 \\
19 \\
19 \\
20 \\
20 \\
20 \\
20 \\
20 \\
21 \\
21 \\
21 \\
21 \\
21 \\
22 \\
22 \\
22 \\
22 \\
22 \\
23 \\
23 \\
23 \\
23 \\
23 \\
24 \\
24 \\
24 \\
24 \\
24 \\
25 \\
25 \\
25 \\
25 \\
25 \\
25\end{array}$ & $\begin{array}{l}3 \\
4 \\
1 \\
2 \\
3 \\
4 \\
1 \\
2 \\
3 \\
4 \\
5 \\
1 \\
2 \\
3 \\
4 \\
5 \\
1 \\
2 \\
3 \\
4 \\
5 \\
1 \\
2 \\
3 \\
4 \\
5 \\
1 \\
2 \\
3 \\
4 \\
5 \\
1 \\
2 \\
3 \\
4 \\
5 \\
6\end{array}$ & $\begin{array}{l}0.75 \\
0.60 \\
1.00 \\
0.90 \\
0.76 \\
0.61 \\
1.00 \\
0.91 \\
0.77 \\
0.62 \\
0.50 \\
1.00 \\
0.91 \\
0.78 \\
0.64 \\
0.51 \\
1.00 \\
0.92 \\
0.79 \\
0.65 \\
0.52 \\
1.00 \\
0.92 \\
0.79 \\
0.66 \\
0.53 \\
1.00 \\
0.92 \\
0.80 \\
0.67 \\
0.55 \\
1.00 \\
0.93 \\
0.81 \\
0.68 \\
0.56 \\
0.45\end{array}$ & $\begin{array}{l}0.83 \\
0.78 \\
0.95 \\
0.89 \\
0.84 \\
0.79 \\
0.95 \\
0.90 \\
0.85 \\
0.80 \\
0.75 \\
0.95 \\
0.90 \\
0.86 \\
0.81 \\
0.76 \\
0.95 \\
0.91 \\
0.86 \\
0.82 \\
0.77 \\
0.96 \\
0.91 \\
0.87 \\
0.83 \\
0.78 \\
0.96 \\
0.92 \\
0.87 \\
0.83 \\
0.79 \\
0.96 \\
0.92 \\
0.88 \\
0.84 \\
0.80 \\
0.76\end{array}$ & $\begin{array}{l}0.89 \\
0.83 \\
1.00 \\
0.95 \\
0.89 \\
0.84 \\
1.00 \\
0.95 \\
0.90 \\
0.85 \\
0.80 \\
1.00 \\
0.95 \\
0.90 \\
0.86 \\
0.81 \\
1.00 \\
0.95 \\
0.91 \\
0.86 \\
0.82 \\
1.00 \\
0.96 \\
0.91 \\
0.87 \\
0.83 \\
1.00 \\
0.96 \\
0.92 \\
0.87 \\
0.83 \\
1.00 \\
0.96 \\
0.92 \\
0.88 \\
0.84 \\
0.80\end{array}$ & $\begin{array}{l}0.97 \\
0.79 \\
1.29 \\
1.18 \\
0.96 \\
0.78 \\
1.28 \\
1.17 \\
0.96 \\
0.78 \\
0.65 \\
1.27 \\
1.16 \\
0.96 \\
0.78 \\
0.65 \\
1.26 \\
1.16 \\
0.91 \\
0.76 \\
0.64 \\
1.25 \\
1.15 \\
0.95 \\
0.78 \\
0.65 \\
1.24 \\
1.14 \\
0.95 \\
0.78 \\
0.65 \\
1.24 \\
1.14 \\
0.95 \\
0.78 \\
0.65 \\
0.55\end{array}$ & $\begin{array}{l}1.16 \\
1.02 \\
1.26 \\
1.26 \\
1.15 \\
1.01 \\
1.25 \\
1.25 \\
1.14 \\
1.01 \\
0.89 \\
1.24 \\
1.24 \\
1.14 \\
1.01 \\
0.88 \\
1.24 \\
1.24 \\
1.04 \\
0.95 \\
0.85 \\
1.23 \\
1.23 \\
1.12 \\
1.00 \\
0.88 \\
1.22 \\
1.22 \\
1.12 \\
1.00 \\
0.88 \\
1.22 \\
1.21 \\
1.11 \\
0.99 \\
0.88 \\
0.77\end{array}$ & $\begin{array}{l}1.09 \\
0.95 \\
1.29 \\
1.22 \\
1.09 \\
0.95 \\
1.28 \\
1.22 \\
1.08 \\
0.94 \\
0.82 \\
1.27 \\
1.21 \\
1.08 \\
0.94 \\
0.82 \\
1.26 \\
1.20 \\
1.00 \\
0.89 \\
0.79 \\
1.25 \\
1.19 \\
1.07 \\
0.94 \\
0.82 \\
1.24 \\
1.19 \\
1.06 \\
0.93 \\
0.82 \\
1.24 \\
1.18 \\
1.06 \\
0.93 \\
0.82 \\
0.72\end{array}$ & $\begin{array}{l}1.90 \\
1.64 \\
2.26 \\
2.20 \\
1.86 \\
1.60 \\
2.22 \\
2.15 \\
1.82 \\
1.57 \\
1.39 \\
2.18 \\
2.11 \\
1.79 \\
1.53 \\
1.36 \\
2.15 \\
2.08 \\
1.67 \\
1.47 \\
1.31 \\
2.11 \\
2.04 \\
1.73 \\
1.48 \\
1.30 \\
2.08 \\
2.01 \\
1.70 \\
1.46 \\
1.28 \\
2.05 \\
1.98 \\
1.68 \\
1.48 \\
1.26 \\
1.13\end{array}$ & $\begin{array}{l}2.09 \\
1.84 \\
2.21 \\
2.30 \\
2.04 \\
1.79 \\
2.17 \\
2.25 \\
2.00 \\
1.75 \\
1.57 \\
2.14 \\
2.21 \\
1.96 \\
1.72 \\
1.53 \\
2.11 \\
2.18 \\
1.78 \\
1.62 \\
1.47 \\
2.07 \\
2.13 \\
1.89 \\
1.66 \\
1.47 \\
2.05 \\
2.10 \\
1.86 \\
1.63 \\
1.45 \\
2.02 \\
2.07 \\
1.83 \\
1.60 \\
1.42 \\
1.29\end{array}$ \\
\hline
\end{tabular}




\begin{tabular}{|c|c|c|c|c|c|c|}
\hline$\hat{M}_{r}$ & $\hat{W}_{r}$ & $\hat{\mathrm{E}}_{\mathrm{r}}$ & $\hat{M}_{r}$ & $\hat{\mathrm{W}}_{r}$ & $\frac{5}{\mathrm{E}}_{\mathrm{r}}$ & $\hat{M}_{r}$ \\
\hline $\begin{array}{l}2.02 \\
1.78 \\
2.26 \\
2.25 \\
1.98 \\
1.74 \\
2.22 \\
2.20 \\
1.94 \\
1.70 \\
1.52 \\
2.18 \\
2.16 \\
1.90 \\
1.66 \\
1.49 \\
2.15 \\
2.11 \\
1.74 \\
1.58 \\
1.43 \\
2.11 \\
2.09 \\
1.83 \\
1.61 \\
1.43 \\
2.08 \\
2.06 \\
1.81 \\
1.58 \\
1.41 \\
2.05 \\
2.03 \\
1.78 \\
1.55 \\
1.38 \\
1.25 \\
1.01\end{array}$ & $\begin{array}{l}4.99 \\
4.56 \\
5.14 \\
5.41 \\
4.81 \\
4.39 \\
5.00 \\
5.24 \\
4.65 \\
4.23 \\
3.96 \\
4.86 \\
5.08 \\
4.51 \\
4.09 \\
3.82 \\
4.78 \\
4.39 \\
4.24 \\
3.92 \\
3.67 \\
4.63 \\
4.82 \\
4.26 \\
3.84 \\
3.57 \\
4.52 \\
4.70 \\
4.15 \\
3.74 \\
3.47 \\
4.42 \\
4.59 \\
4.04 \\
3.64 \\
3.37 \\
3.19\end{array}$ & $\begin{array}{l}5.21 \\
4.77 \\
5.02 \\
5.54 \\
5.02 \\
4.59 \\
4.88 \\
5.37 \\
4.86 \\
4.43 \\
4.14 \\
4.76 \\
5.21 \\
4.71 \\
4.29 \\
3.99 \\
4.68 \\
4.38 \\
4.37 \\
4.08 \\
3.83 \\
4.54 \\
4.93 \\
4.45 \\
4.03 \\
3.74 \\
4.114 \\
4.81 \\
4.33 \\
3.92 \\
3.63 \\
4.35 \\
4.70 \\
4.22 \\
3.82 \\
3.53 \\
3.33\end{array}$ & $\begin{array}{l}5.13 \\
4.71 \\
5.14 \\
5.47 \\
4.95 \\
4.53 \\
5.00 \\
5.30 \\
4.78 \\
4.37 \\
4.09 \\
4.86 \\
5.15 \\
4.64 \\
4.23 \\
3.95 \\
4.78 \\
4.39 \\
4.32 \\
4.03 \\
3.79 \\
4.63 \\
4.87 \\
4.38 \\
3.98 \\
3.70 \\
4.52 \\
4.75 \\
4.26 \\
3.87 \\
3.59 \\
4.42 \\
4.64 \\
4.16 \\
3.77 \\
3.49 \\
3.30\end{array}$ & $\begin{array}{l}15.57 \\
14.85 \\
14.15 \\
16.00 \\
14.88 \\
14.16 \\
13.64 \\
15.37 \\
14.26 \\
13.55 \\
13.14 \\
13.18 \\
14.80 \\
13.71 \\
12.99 \\
12.57 \\
12.78 \\
13.15 \\
12.99 \\
12.43 \\
12.05 \\
12.37 \\
13.81 \\
12.74 \\
12.02 \\
11.60 \\
12.01 \\
13.38 \\
12.32 \\
11.60 \\
11.17 \\
11.68 \\
12.98 \\
11.98 \\
11.21 \\
10.78 \\
10.52\end{array}$ & $\begin{array}{l}15.86 \\
15.10 \\
13.77 \\
16.19 \\
15.16 \\
14.41 \\
13.30 \\
15.56 \\
14.54 \\
13.79 \\
13.33 \\
12.87 \\
14.99 \\
13.98 \\
13.23 \\
12.76 \\
12.47 \\
13.08 \\
13.17 \\
12.64 \\
12.23 \\
12.11 \\
13.99 \\
12.99 \\
12.25 \\
11.79 \\
11.77 \\
13.55 \\
12.56 \\
11.83 \\
11.36 \\
11.46 \\
13.15 \\
12.17 \\
11.43 \\
10.97 \\
10.67\end{array}$ & $\begin{array}{l}15.75 \\
15.02 \\
14.15 \\
16.09 \\
15.06 \\
14.34 \\
13.64 \\
15.47 \\
14.44 \\
13.72 \\
13.28 \\
13.18 \\
14.90 \\
13.88 \\
13.16 \\
12.72 \\
12.78 \\
13.12 \\
13.11 \\
12.58 \\
12.18 \\
12.37 \\
13.90 \\
12.90 \\
12.19 \\
11.74 \\
12.01 \\
13.47 \\
12.48 \\
11.76 \\
11.32 \\
11.68 \\
13.06 \\
12.08 \\
11.37 \\
10.92 \\
10.64\end{array}$ \\
\hline
\end{tabular}


For a given shape parameter, if the values in Table 8 are multiplied by the corresponding values from Table 7, we have an indication of relative efflciency of the censored estimates with respect to the maximum likelihood estimato. That 19

$$
\frac{V^{*}(\hat{m})}{\operatorname{MSE}(x)} \frac{\operatorname{MSE}(\bar{x})}{\operatorname{MSE}(\hat{y})}=\frac{V^{*}(\hat{m})}{\operatorname{MSE}(\hat{x})}
$$

For the once censored case with a sample size of 25 these values are all greater than or equal to one. Thet is, once censored estimators of this type have a lower mean square error than does the maximum likelihood estimate. The gains in efficiency for the once censored case range from about 15 percent for $a=2$ to over 300 percent for $a=5$. From the results of splitstone $(40)$ on the variances of the maximum likelihood estimators of the parameters of the weibull for finite samples we know that the asymptotic variance differs fron the finite result by less than six percent for a sample size of 25. For example splitstone's estimate of the variance of the estimated shape parameter of the Weibull based on a sample of size 25 is .102. while the asymptotic result is .096 . Of course the difference decreases as the sample size increases. This result suggests that for moderate sized samples. alnce the asymptotic variance of the shape parameter approximates that of the true variance, the use of the asymptotic form in place of the true ralue provides a good indlcation of relative efriciency. 
In Chapter IV we considered the estimate $\hat{M}_{0}$, the minimum mean square error estimate of the mean of the exponential distribution

$$
\operatorname{MSE}\left(M_{0}\right)=\left(\frac{c}{n+1}\right)^{2}\left(n \Gamma(2 a+1)-(n-1)(\Gamma(a+1))^{2}\right) .
$$

Recalling that $\operatorname{MSE}(\bar{x})=n^{-1} c^{2}\left(\Gamma(2 a+1)-(\Gamma(a+1))^{2}\right)$, note that as the sample size increases we seo that $R E M\left(\hat{M}_{0}\right)$ approaches unity quite rapidiy. Therefore the use of the REM instead of the relative efficlency based on $\hat{\mathrm{M}}_{0}$ does not have a significant effect on the values of Table 8 .

C. Estimation of the sum of the Largest order statistics We now consider the estimation of the expected value of the sum of the largest two order statistics, $E(Y(n-1)+$ $\left.Y_{(n)}\right)$. Wo shall compare the two estimators $Y_{(n-1)}+Y_{(n)}$ and $2 Y(n-1)$. Thls study will allow us to make some specific statements about the once censored estimators for the mean based on a sample of $n$ observations which are discussed above. We must first develop some preliminary results. We will show that for Weibull order statistics, $Y_{(1)}$ with integer shape parameter, a, larger than one that $C\left(Y_{(n-j)}, Y_{(n)}\right)>C\left(Y_{(n-j)}, Y_{(n-1)}\right)$. We will do this by considering the exponential random variable $X$ such that $X_{(1)}^{a}=$ $Y(1)$ : In Chapter IV we have stated that for the exponential 
distribution $\quad c\left(x_{(n-j)}, x_{(n)}\right)=c\left(x_{(n-j)}, x_{(n-1)}\right)$ since $c\left(x_{(n-j)}, X_{(n-k)}\right)=V(x(n-j))$ if $n-j<n-k$. We now show for $a>1$ that $c\left(x_{(n-j)}^{a}, x_{(n)}^{a}\right)>c\left(x_{(n-j)}^{a}, x_{(n-1)}^{a}\right)$. From Equation 2.19 we have

$$
\begin{aligned}
& x_{(n)}=x_{(n-j)}+w_{n, j} \\
& x_{(n-1)}=x_{(n-j)}+w_{n-1, j-1}
\end{aligned}
$$

and from Equation 2.22

$$
\begin{aligned}
E\left(\left(x_{(n-j)} x_{(n)}\right)^{a}\right) & =E\left(x_{(n-j)}^{a} \sum_{1=0}^{a}\left(\begin{array}{l}
a \\
1
\end{array}\right) x_{(n-j)}^{1} w_{n, j}^{a-1}\right) \\
& =\sum_{1=0}^{a}\left(\begin{array}{l}
a \\
1
\end{array}\right) E\left(x_{(n-j)}^{a+1}\right) E\left(w_{n, j}^{a-1}\right) \\
E\left(\left(x_{(n-j)} X_{(n-1)}\right)^{a}\right) & =\sum_{1=0}^{a}\left(\begin{array}{l}
a \\
1
\end{array}\right) E\left(x_{(n-j)}^{a+1}\right) E\left(w_{n-1, j-1}^{a-1}\right)
\end{aligned}
$$

Hence

$$
\begin{aligned}
& C\left(x_{(n-j)}^{a}, x_{(n)}^{a}\right)=\sum_{1=0}^{a}\left(\begin{array}{l}
a \\
1
\end{array}\right) E\left(X_{(n-j)}^{a+1}\right) E\left(w_{n, j}^{a-1}\right)-e\left(X_{(n-j)}^{a}\right) E\left(x_{(n)}^{a}\right) \\
& =\Sigma^{a}\left(\begin{array}{l}
a \\
1
\end{array}\right) E\left(x_{(n-j)}^{a+1}\right) E\left(w_{n, j}^{a-1}\right)-E\left(x_{(n-j)}^{a}\right) \Sigma\left(\begin{array}{l}
a \\
1
\end{array}\right) E\left(x_{(n-j)}^{1}\right) E\left(w_{n, j}^{a-1}\right) \\
& =\stackrel{a}{\Sigma}\left(\begin{array}{l}
a \\
1
\end{array}\right)\left(E\left(x_{(n-j)}^{a+1}\right)-E\left(x_{(n-j)}^{a}\right) E\left(x_{(n-j)}^{1}\right)\right) E\left(w_{n, j}^{a-1}\right) \text {. }
\end{aligned}
$$

Similarly

$$
\begin{aligned}
C\left(x_{(n-j)}^{a}, x_{(n-1)}^{a}\right) & =\sum^{a}\left(\begin{array}{l}
a \\
1
\end{array}\right)\left(E\left(x_{(n-j)}^{a+1}\right)\right. \\
& \left.-E\left(x_{(n-j)}^{a}\right) E\left(x_{(n-j)}^{1}\right)\right) E\left(w_{n-1, j-1}^{a-1}\right) .
\end{aligned}
$$


Hence $C(Y(n-j), Y(n))>C(Y(n-j), Y(n-1))$ if $E\left(W_{n, j}^{a-1}\right)-$ $E\left(w_{n-1 ; j-1}^{a-1}\right)>0$, but

$$
w_{n, j}=x_{(n)}-x_{(n-j)}>x_{(n-1)}-x_{(n-j)}=w_{n-1, j-1}
$$

since $X_{(n)}>X_{(n-1)}$. Therefore we have the desired result.

Lemma: For $Y$ a Welbull random variable with integer shape parameter a> 1

$$
C(Y(n-j), Y(n))>C(Y(n-j), Y(n-1))
$$

for $j \geqslant 1$.

From Equation 5.27 we write

$$
\begin{aligned}
& C\left(Y_{(n-j)}, Y_{(n)}\right)-C\left(Y_{(n-j)}, Y_{(n-1)}\right)>0 \\
& E\left(Y_{(n-j)}\left(Y_{(n)}-Y_{(n-1)}\right)\right)-E\left(Y_{(n-j)}\right) E\left(Y_{(n)}-Y_{(n-1)}\right)>0 \\
& C\left(Y_{(n-j)}, Y_{(n)}-Y_{(n-1)}\right)>0 \quad j \geqslant 1 .
\end{aligned}
$$

That is, the covariance of any lower weibull order statistic with the difference $Y(n)-Y(n-1)$ is positive.

We now investigate the variance of certain linear combinations of order statistics. To facilitate the discussion we introduce the nxI vectors

$$
\begin{aligned}
& A_{1}^{\prime}=(1, \ldots, 1,1,1) \\
& A_{2}^{\prime}=(1, \ldots, 1,2,0)
\end{aligned}
$$




$$
\begin{aligned}
A_{3}^{\prime} & =(0, \ldots, 0,-1,1) \\
B_{1}^{\prime} & =(0, \ldots, 0,1,1) \\
B_{2}^{\prime} & =(0, \ldots, 0,2,0) \\
D^{\prime} & =(1, \ldots, 1,0,0) .
\end{aligned}
$$

Then $A_{1}=A_{2}+A_{3}, B_{1}=B_{2}+A_{3}, A_{1}=D+B$, and $A_{2}=D+B_{2}$. Let $Y$ be the $n \times l$ vector of Welbull order statistics and rocsll $\mathrm{V}(\mathrm{Y})=\$$ and $E(Y)=\varepsilon$. The nXn matrix $\$$ and the $n \times I$ vector $\varepsilon$ are known from the results of Chapter II. Hence

$$
\begin{aligned}
& A_{1}^{\prime} Y=\sum^{n-1} Y(1)+Y(n) \\
& A_{2}^{\prime} Y=\sum^{n-1} Y(1)+Y(n-1) \\
& B_{1}^{\prime} Y=Y(n-1)+Y(n) \\
& B_{2}^{\prime} Y=2 Y(n-1) \\
& D^{\prime} Y=\sum-\sum Y(1) .
\end{aligned}
$$

It can be easily shown by using the matrices of Equation 5.29 and the result in Equation 5.28 that for Welbulls w1th a>1

$$
\begin{aligned}
0< & V(Y(n-1)+Y(n))-V\left(2 Y(n-1)<V\left({ }^{n-1} \Sigma^{Y} Y(1)+Y(n)\right)\right. \\
& -V\left(\stackrel{n-1}{\Sigma} Y_{(1)}+Y(n-1)\right) .
\end{aligned}
$$

We will estimate the mean of the Welbull by estimating the 
$\operatorname{sum} E\left(\sum^{n} Y(1)\right)$ with $Y_{(1)}+\ldots+2 Y(n-1)$. The $\operatorname{sum} Y(1)+\ldots$ $+Y_{(n)}$ of observations is unbiased for $\mathbf{E}\left(\sum^{n} Y(1)\right)$. We w111 compare the mean square errors of the two estimates. Now

$$
\begin{aligned}
& \operatorname{MSE}\left(\sum^{n-1} Y_{(1)}+Y_{(n)}\right)-\operatorname{MSE}\left(\sum^{n-1} Y_{(1)}+Y_{(n-1)}\right)= \\
& A_{1}^{\prime}+A_{1}-A_{2}^{\prime} \downarrow A_{2}-\left(\left(A_{2}-A_{1}\right) \cdot \varepsilon\right)^{2} \\
& =\left(A_{2}+A_{3}\right) \cdot \frac{1}{4}\left(A_{2}+A_{3}\right)-A_{2}^{\prime} \ddagger A_{2}-\left(A_{3}^{\prime} \varepsilon\right)^{2} \\
& =A_{3}^{\prime} \ddagger A_{3}+2 A_{2}^{\prime} \ddagger A_{3}-\left(A_{3}^{\prime} \varepsilon\right)^{2} \text {. }
\end{aligned}
$$

We estimate the sum $\mathbb{B}\left(Y_{(n-1)^{*} Y(n)}\right.$ with $2 . Y(n-1) \cdot$ The sum $Y(n-1)+Y(n)$ of observations is unblased for $E(Y(n-1)+Y(n))$. We w1ll compare the mean square errors of the two estimates.

$$
\begin{aligned}
& \operatorname{MSE}\left(Y_{(n-1)}+Y(n)\right)-\operatorname{MSE}(2 Y(n-1))= \\
& B_{1}^{\prime} \$ B_{1}-B_{2}^{\prime} \$ B_{2}-\left(\left(B_{2}-B_{1}\right) \cdot \varepsilon\right)^{2} \\
& =\left(B_{2}+A_{3}\right) \frac{1}{4}\left(B_{2}+A_{3}\right)-B_{2}^{\prime} B_{2}=\left(A_{3}^{\prime} \varepsilon\right)^{2} \\
& =A_{3}^{\prime} \$ A_{3}+2 B_{2}^{\prime} \$ A_{3}-\left(A^{\prime} \varepsilon\right)^{2} .
\end{aligned}
$$

Hence the difference of the left-hand sides of Equations 5.32 and 5.33 is $2\left(A_{2}-B_{2}\right) ' \$ A_{3}$. But

$$
\begin{aligned}
2\left(A_{2}-B_{2}\right){ }^{\prime} \$ A_{3} & =2 D^{\prime} \$ A_{3} \\
& =2 C\left({ }^{n-2} \Sigma Y(1), Y(n)-Y(n-1)\right) .
\end{aligned}
$$


From Equation 5.28 this covarlance is positive. Then we have

$$
\begin{aligned}
& \operatorname{MSE}\left(Y_{(n-1)}+Y_{(n)}\right)-\operatorname{MSE}(2 Y(n-1))<\operatorname{MSE}\left(\sum Y_{(1)}{ }^{n-1}+Y_{(n)}\right) \\
& -\operatorname{MSE}\left(\sum Y_{(1)}+Y_{(n-1)}\right)
\end{aligned}
$$

We would wish to show that the difference in Equation 5.33 is positive. Unfortunately this result is extremely difficult to prove. The covariance $2 B_{2} \frac{1}{4} A_{3}$ is positive from Equation 5.28 for $a>1$. If $A_{3}^{\prime} t^{\prime} A_{3}>\left(A_{3} \varepsilon\right)^{2}$ we would have the desired result. That is if $V\left(Y_{(n)}-Y_{(n-1)}\right)>\left(E\left(Y_{(n)}-\right.\right.$ $\left.Y_{(n-1)}\right)^{2}$ we would have the difference in 5.33 being positive. Under this assumption we would have, taking the square root of both sides $\sqrt{V(Y(n)-Y(n-1)}) / E\left(Y_{(n)}-Y_{(n-1)}\right)>1$. But this is exactly the coefficient of variation of $Y(n)-Y(n-1))$. Hence if the coefficient of varlation were greater than one the right-hand side of Equation 5.33 would be positive. For the exponential distribution it is known that the coefficient of varlation is one. Now for the welbull with shape parameter asl it is heuristicly clear that the difference $x_{(n)}^{a}-x_{(n-1)}^{a}$ would be more variable than $x_{(n)}-X_{(n-1)}$ since the distr1bution is more skewed and hence the coefficient of variation would be greater than one. This is verified for small samples. Now we are able to show that the left-hand side of Equation 5.34 is positive for the larger sample sizes. We shall do thio using the asymptotic theory developed in 
Chapter III. In particular we use a speclal case of Equation 3.28 , name ly

$$
E^{*}\left(X_{(n-1)}^{m}\right)=E^{*}\left(X_{(n)}^{m}\right)-m E^{*}\left(X_{(n)}^{m-1}\right)
$$

Now we have already stated that $Y_{(n-1)}+Y_{(n)}$ estimates $E\left(Y_{(n-I)}+Y_{(n)}\right)$ unbiasedly. However $2 Y_{(n-1)}$ is blased for $\mathrm{E}\left(\mathrm{Y}_{(n-1)}+\mathrm{Y}_{(\mathrm{n})}\right)$ and the b1as is

$$
\begin{aligned}
& E^{*}\left(x_{(n-1)}^{a}-X_{(n)}^{a}\right)=E^{*}\left(x_{(n-1)}^{a}\right)-E^{*}\left(X_{(n)}^{a}\right) \\
& =E^{*}\left(x_{(n)}^{a}\right)-a E^{*}\left(x_{(n)}^{a-1}\right)-E^{*}\left(x_{(n)}^{a}\right) \\
& =-a E^{*}\left(x_{(n)}^{a-1)}\right. \\
& \left(\text { Bias }\left(2 y_{(n-1)}\right)\right)^{2}=a^{2} E^{*}\left(x_{(n)}^{a}-1\right) .
\end{aligned}
$$

Next we find the asymptotic variance of the random variable $2 Y(n-1)$

$$
\begin{aligned}
& V^{*}(2 Y(n-1))=V^{*}\left(2 X_{(n-1)}^{a}\right)=4\left(E^{*}\left(x_{(n-1)}^{2 a}\right)-E^{*}\left(x_{(n-1)}^{a}\right)(5 \cdot 38)\right. \\
& =4\left(E^{*}\left(X_{(n)}^{2 a}\right)-2 a E^{*}\left(x_{(n)}^{2 a-1}\right)-\left(E^{*}\left(x_{(n)}^{a}\right)-a E^{*}\left(x_{(n)}^{a-1}\right)\right)^{2}\right) \\
& =4 E^{*}\left(X_{(n)}^{2 a}\right)-8 a E^{*}\left(x_{(n)}^{2 a-1}\right)-4 E^{*}\left(x_{(n)}^{a}\right) \\
& \quad-4 a^{2} E^{* 2}\left(x_{(n-1)}^{a-1}\right)+8 a E^{*}\left(x_{(n)}^{a}\right) E^{*}\left(x_{(n-1)}^{a}\right) .
\end{aligned}
$$

Hence the asymptotic mean square error of $2 Y_{(n-1)}$ is $\operatorname{MSE}^{*}(2 Y(n-1))=4 E^{*}\left(X_{(n)}^{2 a}\right)-8 a E^{*}\left(x_{(n)}^{2 a-1}\right)-4 E^{*}\left(x_{(n)}^{a}\right)$

$$
-3 a^{2} E^{*}\left(x_{(n)}^{a-1}\right)+8 a E^{*}\left(x_{(n)}^{a}\right) E^{*}\left(x_{(n)}^{a-1}\right) \text {. }
$$


Now the approximate asymptotic varlance and thus the mean square error of $Y(n-1)+Y(n)$ is

$$
\begin{aligned}
& \operatorname{MSE}^{*}\left(Y_{(n-1)}\right.\left.Y_{(n)}\right)=V^{*}\left(X_{(n-1)}^{a}\right)+V^{*}\left(X_{(n)}^{a}\right)+2 C^{*}\left(X_{(n-1)}^{a}, X_{(n)}^{a}\right) \\
&=E^{*}\left(X_{(n-1)}^{2 a}\right)-E^{*}\left(X_{(n-1)}^{a}\right)+E^{*}\left(X_{(n)}^{2 a}\right)-E^{2}\left(X_{(n)}^{a}\right) \\
&+2\left(E^{*}\left(X_{(n-1)}^{a} X_{(n)}\right)-E^{*}\left(X_{(n)}^{a}\right) E^{*}\left(X_{(n-1)}^{a}\right)\right) \\
&=2 E^{*}\left(X_{(n)}^{2 a}\right)-2 a E^{*}\left(X_{(n)}^{2 a-1}\right)-4 E^{*}\left(X_{(n)}^{a}\right)-a^{2} E^{*}\left(X_{(n)}^{a-1}\right) \\
& \\
& \quad+4 a E^{*}\left(X_{(n)}^{a}\right) E^{*}\left(X_{(n)}^{a}\right)+2 E^{*}\left(X_{(n-1)}^{a} X_{(n)}^{a}\right)
\end{aligned}
$$

From Equation 3.30 and using the fact that $E\left(w_{n, 1}^{a-1}\right)=(a-1)$ ! we have

$$
\begin{aligned}
& E^{*}\left(x_{(n-1)}^{a} x_{(n)}^{a}\right)=\sum^{a} \frac{a !}{1 !} E^{*}\left(x_{(n-1)}^{a+1}\right) \\
& \quad=\sum \frac{a !}{1 !}\left(E^{*}\left(x_{(n)}^{a+1}\right)-(a+1) E^{*}\left(x_{(n)}^{a+1-1}\right)\right) .
\end{aligned}
$$

Now the highest two 1ridices of 5.41 contain the highest moments of $x(n)$. Substituting 5.41 into Equation 5.40 we write

$$
\begin{aligned}
\operatorname{MSE}^{*}(Y(n-1) & Y(n))=4 E^{*}\left(X_{(n)}^{2 a}\right)-4 a E^{*}\left(X_{(n)}^{2 a-1}\right) \\
& -2(2 a-1) E^{*}\left(X_{(n)}^{2 a-2}\right)-4 E^{*}\left(X_{(n)}^{a}\right)-a^{2} E^{*}\left(X_{(n)}^{a-1}\right) \\
& +4 a E^{*}\left(X_{(n)}^{a}\right) E^{*}\left(X_{(n)}^{a-1}\right) \\
& +2 a !^{a-2}\left(E^{*}\left(X_{(n)}^{a+1}\right)-(a+1) E^{*}\left(X_{(n)}^{a+1-1}\right)\right) / 1 !
\end{aligned}
$$


Now taking the difference of Equations 5.41 and 5.42

$$
\begin{aligned}
& \operatorname{MSE}^{*}\left(Y_{(n-1)}+Y_{(n)}\right)-\operatorname{MSE}^{*}(2 Y(n-1)) \\
& =4 a E^{*}\left(X_{(n)}^{2 a-1}\right)-(4 a-2) E^{*}\left(X_{(n)}^{2 a-2}\right)+2 a^{2} E^{2}\left(X_{(n)}^{a-1}\right) \\
& -4 a E^{*}\left(X_{(n)}^{a}\right) E^{*}\left(X_{(n)}^{a}-1\right)+2 a ! \stackrel{a-2}{\Sigma}\left(E^{*}\left(X_{(n)}^{a+1}\right)\right. \\
& \left.-(a+1) E^{*}\left(X_{(n)}^{a+1-1}\right)\right) / 1 !
\end{aligned}
$$

The difference has, as we would expect, a value of zero for a equal to one. Substituting Equation 3.27 into Equation 5.43 we see that the difference $\operatorname{MSE}^{*}\left(Y_{(n-1)}+\right.$ $Y(n))-\operatorname{MSE}^{*}(2 Y(n-1))$ is expressible as a polynomial in log $n$ since $E^{*}\left(X_{(n)}^{m}\right)=\sum_{1=0}^{m}\left(\begin{array}{l}m \\ 1\end{array}\right)(\log n)^{m-1} I(1)$. Study of Equation 5.43 shows us that the highest possible degree of this polynomlal is $2 a-1$. However if we separate the two terms of this degree, letting $R_{(1)}$ be a polynomial of degree 1 in $\log n$, we have

$$
\begin{aligned}
& 4 a\left(E^{*}\left(X_{(n)}^{2 a-1}\right)-E^{*}\left(X_{(n)}^{a}\right) E^{*}\left(X_{(n)}^{a-1}\right)\right)=4 a\left((\log n)^{2 a-1}\right. \\
& +(2 a-1) I(1)(\log n)^{2 a-2}+R(2 a-3)-\left((\log n)^{a}\right. \\
& \left.\quad+a I(1)(\log n)^{a-1}+R(a-2)\right)\left((\log n)^{a-1}\right. \\
& \left.\quad+(a-1) I(1)(\log n)^{a-2}+R(a-3)\right) \\
& =4 a\left((\log n)^{2 a-1}+(2 a-I) I(1)(\log n)^{2 a-2}-\log n\right)^{2 a-1} \\
& \left.\quad-a I(1)(\log n)^{2 a-2}-(a-1) I(I)(\log n)^{2 a-2}+R(2 a-3)\right)
\end{aligned}
$$




$$
=4 a(R(2 a-3))
$$

where $R(2 a-3)=0$ if $2 a-3<0$.

Hence the degree of the polynomial is $2 a-2$; Collecting the terms in 5.43 which have degree $2 a-2$; we have

$$
\begin{gathered}
-(4 a-2) E^{*}\left(X_{(n)}^{2 a-2}\right)+2 a^{2} E^{2}\left(X_{(n)}^{a-1}\right)+2 a(a-1) E^{*}\left(X_{(n)}^{2 a-2}\right)= \\
\left(4 a^{2}-6 a+2\right)(\log n)^{2 a-2}+R(2 a-3)
\end{gathered}
$$

Then we are able to write Equation 5.43 as

$$
\begin{aligned}
& \operatorname{MSE}^{*}(Y(n-1)+Y(n))-\operatorname{MSE}^{*}(2 Y(n-1))= \\
& \left(4 a^{2}-6 a+2\right)(\log n)^{2 a-2}+R(2 a-3)
\end{aligned}
$$

where $Y$ is a Weibull with shape parameter a. Now due to the binomial form of $E\left(X_{(n)}^{m}\right)$ we know that the terms of $R(2 a-3)$ are of the form $c_{1}(\log n)^{2 a-2-1}, 1=1,2, \ldots$, 2a-2 where $c_{1}$ are polynomials in a and the constants $I(j)$. The values of $I(J)$ are in Table 4. Hence the right-hand side of Equation 5.44 is a continuous function in a. The derivative of this function is

$$
\begin{aligned}
& (8 a-6)(\log n)^{2 a-2}+2(4 a-6 a+2)(\log (\log n))(\log n)^{2 a-2} \\
& +\sum_{1=1}^{2 a-2} \frac{d}{d a} c_{1}(\log n)^{2 a-2-1} \text {. } \\
& \text { Since the leading coefficient of the right-hand side } \\
& \text { of Equation } 5.43 \text { is positive for } a>1 \text { and the leading }
\end{aligned}
$$


coefficient of the derivative is positive for $a>1$ we conclude that for large sample sizes, the difference in Equation 5.43 is an increasing function in a and is positive for $a>1$. We are only able to check integer values of a, for example for $a=2$

$$
\begin{aligned}
\operatorname{MSE}^{*}\left(Y_{(n-1)}+Y_{(n)}\right)-\operatorname{MSE}^{*}(2 Y(n-1) & =8 E^{*}\left(X_{(n)}^{3}\right) \\
& -2 E^{*}\left(X_{(n)}^{2}\right)+8\left(E^{2}(X(n))-E^{*}\left(X_{(n)}^{2}\right)-E^{*}\left(X_{(n)}\right)\right) \\
= & 6(\log n)^{2}+25.27 \log n+32.8>0 .
\end{aligned}
$$

Now from Equations 5.44 and 5.45 we conclude that

$$
\operatorname{MSE}^{*}\left(Y_{(n-1)}+Y_{(n)}\right)-\operatorname{MSE}^{*}(2 Y(n-1))>0
$$

for a $>1$. Therefore from Equation 5.34

$$
\operatorname{MSE}(\stackrel{n-1}{\Gamma} Y(1)+Y(n-1))<\operatorname{MSE}\left(\stackrel{n-1}{\Gamma} Y_{(1)}+Y_{(n)}\right)
$$

and

$$
\operatorname{MSE}\left(\hat{W}_{1}\right)<\operatorname{MSE}(\bar{Y})
$$

for large $n$. We have already shown that 5.47 is true for samples of size up to 25 . Hence we conclude that 5.47 is true for all values of $n$.

Then we have the relative efficiency with respect to the mean 
$\operatorname{REM}\left(\hat{W}_{1}\right)=\frac{\operatorname{MSE}(\bar{Y})}{\operatorname{MSE}\left(\hat{W}_{1}\right)}=1+\frac{\operatorname{MSE}(\bar{Y})-\operatorname{MSE}\left(\hat{W}_{1}\right)}{\operatorname{MSE}\left(\hat{W}_{1}\right)} \geqslant 1$

since the $\operatorname{MSE}\left(\hat{W}_{1}\right)$ is positive and expressible using Equations 3.27 and 3.28 as a polynomial in $\log n$ of degree $2 a$. Hence the quotient on the right-hand side of Equation 5.48 approaches: zero as $n$ increases without bound since the highest power of $\log \mathrm{n}$ in the numerator is $2 a-2$. We have already noted that equality also occurs if a is equal to one.

D. Tests for Departure from the Exponential

It is known that the distribution of the $k$-th order statistic in a sample of size $n$ from a distribution with distribution function $F$ and density function $f$ is

$$
f_{X(k)}(x)=k\left(\begin{array}{l}
n \\
k
\end{array}\right) F(x)^{k-1}(1-F(x))^{n-k_{f}} f(x) d x
$$

Hence the distribution of the $k$-th order statistic of a standard exponential is

$$
f_{x(k)}(x)=k\left(\begin{array}{l}
n \\
k
\end{array}\right)\left(1-e^{-x}\right)^{k-1} e^{-x(n-k)} e^{-x} d x
$$

Using the binomial theorem on Equation 5.50 gives us

$$
f_{X(k)}(x)=k\left(\begin{array}{l}
n \\
k
\end{array}\right) \sum_{1=0}^{k-1}\left(\begin{array}{c}
k-1 \\
1
\end{array}\right)(-1)^{1} e^{(1+n-k+1) x} d x
$$

Now from Fuller (18) if $\Delta$ is a small number

$$
\int x^{(1+\Delta)} e^{-a x} d x=\int\left(a^{-1} y\right)^{1+\Delta} e^{-y} a^{-1} d y
$$


where $\mathrm{y}=\mathrm{ax}$. Hence

$$
\int_{x}(1+\Delta) e^{-a x} d x=a^{-2-\Delta} \Gamma(2+\Lambda) \text {. }
$$

Furthermore if we expand the result of Equation 5.52 as a Inear function of $\wedge$ we have

$a^{-2-\Delta} \Gamma(2+\Delta) \doteq a^{-2} \Gamma(2)-a^{-2}(\log a) \Gamma(2) \Delta+a^{-2} \Gamma(1)(2) \wedge$

where $\Gamma$ is the gamma function. Hence $\Gamma(2)=1$ and $\Gamma(1)(2)=$ .4227. From Equation 5.52 the expected value of the $k$-th standard exponential order statistic to the power $1+\Lambda$ is

$$
\begin{aligned}
E\left(X_{(k)}^{1+\Lambda}\right) & =\int x^{1+\Delta} f_{X}(x) d x \\
& =k\left(\begin{array}{l}
n \\
k
\end{array}\right) \sum_{1=0}^{k-1}\left(\begin{array}{l}
k \\
1
\end{array}\right)(-1)^{1}(1+n-k+1)^{-2-\Delta} \Gamma(2+1) .
\end{aligned}
$$

Using the approximation of Equation 5.53 we write Equation 5.54 as

$$
\begin{gathered}
E\left(X_{(k)}^{1+\Delta}\right) \doteq k\left(\begin{array}{l}
n \\
k
\end{array}\right) \sum_{1=0}^{k-1}\left(\begin{array}{c}
k-1 \\
1
\end{array}\right)(-1)^{1}\left((1+n-k+1)^{-2}\right. \\
(1+(-\log (1+n-k+1)+.4227) \Delta)) .
\end{gathered}
$$

Letting

$$
A_{k}=\sum_{1=0}^{k-1} 1 / n-1
$$

and

$$
B_{k}=k\left(\begin{array}{l}
n \\
k
\end{array}\right) \sum_{1=0}^{k-1}\left(\begin{array}{c}
k-1 \\
1
\end{array}\right)(-1)^{1}(1+n-k+1)^{-2} \log (1+n-k+1)
$$


so we write Equation 5.55 as

$$
E\left(X_{(k)}^{I+\Delta}\right)=A_{k}+\left(.4227 A_{k}-B_{k}\right) \Delta
$$

Recall Equation 1.1 and consider the random varlables

$$
z_{k}=(n-k+1)\left(x_{(n-k+1)}^{1+\Delta}-x_{(n-k)}^{1+\Delta}\right)
$$

to be approximately uncorrelated. Then

$$
E\left(z_{k}\right) \neq 1+\left(.4227+D_{k}\right) \Delta
$$

where

$$
\begin{aligned}
& D_{k}=-(n-k+1)\left(B_{k}-B_{k-1}\right) \\
& D_{k}=-(n-k+1)\left(\begin{array}{c}
n \\
k-1
\end{array}\right) \sum_{1=0}^{k-1}\left(\begin{array}{c}
k-1 \\
1
\end{array}\right)(-1)^{1} \frac{\log (1+n-k+1)}{(1+n-k+1)} \cdot(5,59)
\end{aligned}
$$

The $D_{k}$ are increasing with $k$ and sum to zero. The values for $D_{k}$ for sample sizes from 2 to 30 are found in Table 9. Suppose there is an initial estimate for a say $a_{0}$. Write the variables $z_{k}$ as

$$
Z_{k}=(n-k+1)\left(Y_{(k)}^{1 / a_{0}}-Y_{(k-1)}^{1 / a_{0}}\right)
$$

where $Y(0)$ is understood to be zero, Now let $1 / a_{0}=$ $(1 / a)(1+\Delta)$ and hence from Equation 5.58

$$
E\left(z_{k}\right)=c^{1 / a_{0}}+\left(.4227+D_{k}\right) c^{1 / a_{0}} \Delta .
$$

Therefore 1 t 13 possible to estimate $c^{1 / a}$ and $c^{1 / a} \Delta$ by the 
TAELE 9.

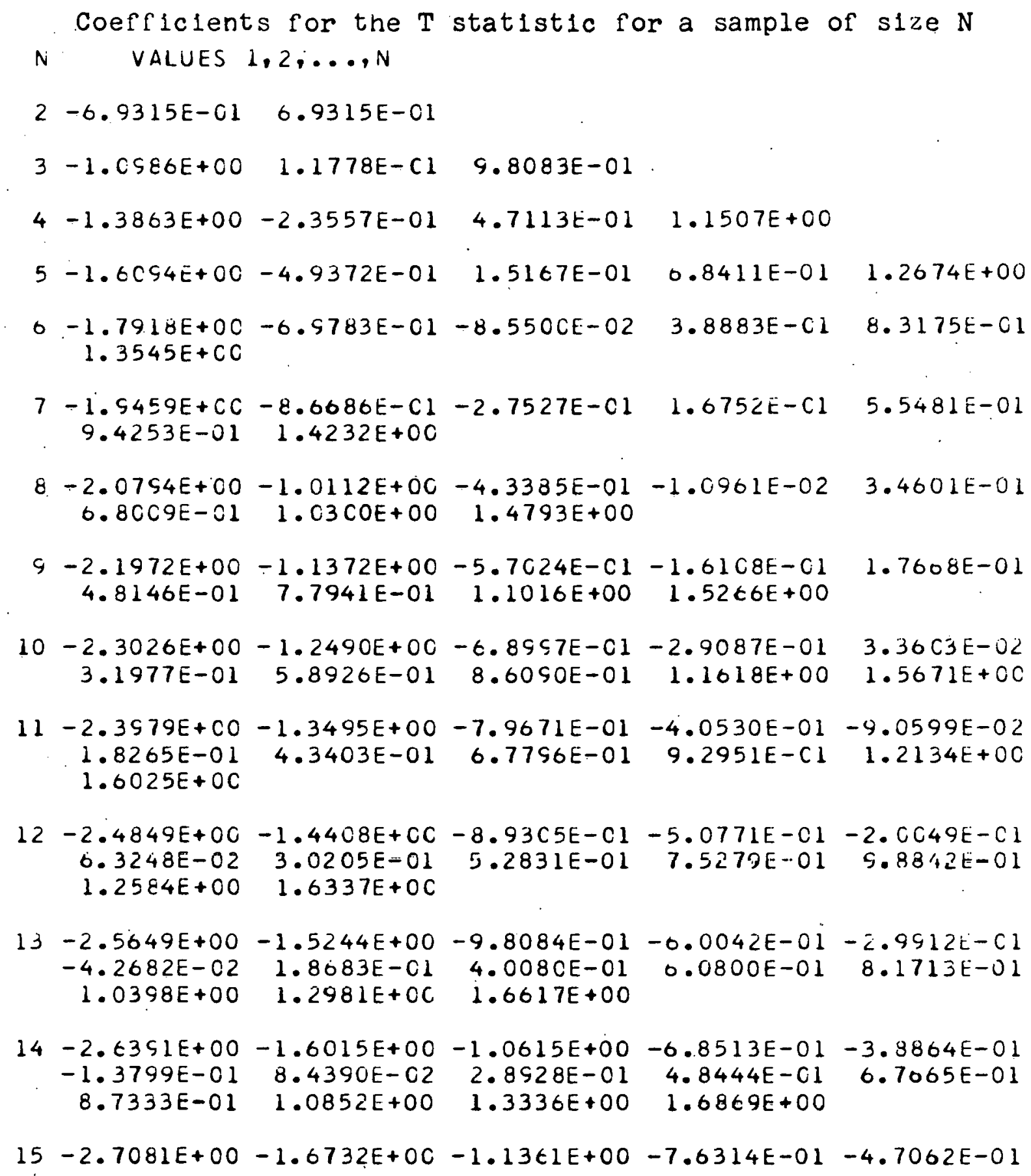




\section{TABLE 9. (CONT INUEC)}

\begin{tabular}{|c|c|c|c|c|c|}
\hline & $\begin{array}{r}-2.2467 E-01 \\
7.3667 E-01\end{array}$ & $\begin{array}{r}-7.9598 E-03 \\
9.2302 E-01\end{array}$ & $\begin{array}{l}1.8953 E-C 1 \\
1.1257 E+00\end{array}$ & $\begin{array}{l}3.7620 E-C 1 \\
1.3656 E+00\end{array}$ & $9 E+00$ \\
\hline 6 & $\begin{array}{r}-2.7726 E+C 0 \\
-3.0421 E-01 \\
6.1578 E-C 1 \\
1.7309 E+00\end{array}$ & $\begin{array}{r}-1.740 C E+00 \\
-9.2107 E-02 \\
7.8981 E-01\end{array}$ & $\begin{array}{r}-1.2055 E+C 0 \\
1.0023 E-01 \\
9.6743 E-01\end{array}$ & $\begin{array}{r}-8.3543 E-01 \\
2.7964 E-01 \\
1.1623 E+00\end{array}$ & $\begin{array}{r}-5.462 \\
4.513 \\
1.39\end{array}$ \\
\hline 7 & $\begin{array}{r}-2.8332 E+00 \\
-3.7772 E-C 1 \\
5.1714 E-01 \\
1.4212 E+C O\end{array}$ & $\begin{array}{r}-1.8026 E+00 \\
-1.6944 E-01 \\
6.7577 E-01 \\
1.7503 E+00\end{array}$ & $\begin{array}{r}-1.27 C \\
1.837 \\
8.373\end{array}$ & $\begin{array}{r}-5.0280 E-C .1 \\
1.9232 E-01 \\
1.0075 E+C 0\end{array}$ & $\begin{array}{r}-6 \cdot 1 \\
3 \cdot 5 \\
1.1\end{array}$ \\
\hline 8 & $\begin{array}{r}-2.8904 E+00 \\
-4.46 C 7 E-01 \\
4.2537 E-01 \\
1.2258 E+0 C\end{array}$ & $\begin{array}{r}-1.86 \\
-2.41 \\
5.75 \\
1.44\end{array}$ & $\begin{array}{r}-1.3312 E+C 0 \\
-5.6964 E-02 \\
7.2588 E-01 \\
1.7682 E+00\end{array}$ & $\begin{array}{r}-9.6587 E-01 \\
1.1254 E-01 \\
8.8019 E-01\end{array}$ & $\begin{array}{r}-6.8203 E-01 \\
2.7210 E-C 1 \\
1.0438 E+00\end{array}$ \\
\hline 19 & $\begin{array}{r}-2.9444 E+00 \\
-5.0995 E-01 \\
3.4220 E-01 \\
1.0771 E+00\end{array}$ & $\begin{array}{r}-1.9172 E+00 \\
-3.0766 E-01 \\
4.8585 E-01 \\
1.2537 E+00\end{array}$ & $\begin{array}{r}-1.3886 E+00 \\
-1.2678 E-01 \\
6.2786 E-C 1 \\
1.4682 E+00\end{array}$ & $\begin{array}{r}-1.0252 E+00 \\
3.9029 E-02 \\
7.7111 E-C 1 \\
1.7849 E+00\end{array}$ & $\begin{array}{r}-7.4349 E-01 \\
1.9422 E-01 \\
5.1914 E-01\end{array}$ \\
\hline 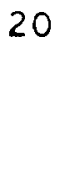 & $\begin{array}{r}-2.9957 E+00 \\
-5.0992 E-01 \\
2.66 C 5 E-01 \\
9.5478 E-01\end{array}$ & $\begin{array}{r}-1.96 \\
-3.70 \\
4.04 \\
1.10\end{array}$ & $\begin{array}{r}-1.44 \\
-1.91 \\
5.40 \\
1.27\end{array}$ & $\begin{array}{r}-1.0811 E+00 \\
-2.9168 E-02 \\
6.7513 E-01 \\
1.4892 E+00\end{array}$ & $\begin{array}{l}4 E-01 \\
8 E-01 \\
5 E-01 \\
4 E+C C\end{array}$ \\
\hline 21 & $\begin{array}{r}-3.0445 E+00 \\
-6.2644 E-01 \\
1.9576 E-01 \\
8.4989 E-C 1 \\
1.8150 E+00\end{array}$ & $\begin{array}{r}-2.0199 E+00 \\
-4.2862 E-01 \\
3.2996 E-01 \\
9.8755 E-01\end{array}$ & $\begin{array}{r}-1.4943 E+00 \\
-2.5281 E-C 1 \\
4.6041 E-01 \\
1.1355 E+C O\end{array}$ & $\begin{array}{r}-1.1341 E+00 \\
-5.2798 E-C 2 \\
5.8911 E-01 \\
1.3034 E+00\end{array}$ & $\begin{array}{l}9 E-01 \\
3 E-0.2 \\
4 E-01 \\
7 E+0.0\end{array}$ \\
\hline 2 & $\begin{array}{r}-3.0910 E+00 \\
-6.7589 E-01 \\
1.3043 E-01 \\
7.5752 E-01 \\
1.5270 E+00\end{array}$ & $\begin{array}{r}-2.0676 E+00 \\
-4.8391 E-01 \\
2.6109 E-01 \\
8.8454 E-C 1 \\
1.8287 E+00\end{array}$ & $\begin{array}{r}-1.5432 E+00 \\
-3.1015 E-C 1 \\
3.8735 E-01 \\
1 . C 179 E+C O\end{array}$ & $\begin{array}{r}-1.1844 E+00 \\
-1.524 E E-C 1 \\
5.1099 E-C 1 \\
1.162 .1 E+00\end{array}$ & $\begin{array}{r}-5.0775 E-01 \\
-6.6233 E-03 \\
6.3375 E-C 1 \\
1.3257 E+00\end{array}$ \\
\hline 23 & $\begin{array}{r}-3.1355 E+C O \\
-7.3059 E-01 \\
6.9370 E-02\end{array}$ & $\begin{array}{r}-2.1131 E+00 \\
-5.3624 E-01 \\
1.9704 E-01\end{array}$ & $\begin{array}{r}-1.5898 E+00 \\
-3.642 \mathrm{SE}-01 \\
3.1981 \mathrm{E}-\mathrm{Cl}\end{array}$ & $\begin{array}{r}-1.2323 E+C O \\
-2.0863 E-C 1 \\
4.3931 E-01\end{array}$ & $\begin{array}{r}-5.5701 E-C 1 \\
-6.5080 E-02 \\
5.57 C 7 E-C 1\end{array}$ \\
\hline
\end{tabular}


TABLE 9. (CCNTINUEO)

\begin{tabular}{|c|c|c|c|c|c|}
\hline & $\begin{array}{l}6.7465 E-C 1 \\
1.3465 E+00\end{array}$ & $\begin{array}{l}7.9377 E-01 \\
1.5442 E+00\end{array}$ & $\begin{array}{l}9.1657 E-01 \\
1.8417 E+00\end{array}$ & $1.0460 E+00$ & $6 E+00$ \\
\hline 4 & $\begin{array}{r}-3.1781 E+0 C \\
-7.7882 E-C 1 \\
1.2034 E-02 \\
5.5931 E-01 \\
1.2 C 54 E+C 0\end{array}$ & $\begin{array}{r}-2.1506 E+00 \\
-5.8593 E-01 \\
1.3713 E-01 \\
7.1232 E-01 \\
1.3661 E+0 C\end{array}$ & $\begin{array}{r}-1 \cdot 6 \\
-4 \cdot 1 \\
2.5 \\
8.2 \\
1 \cdot 5\end{array}$ & $\begin{array}{r}-1.2780 E+00 \\
-2.6171 E-01 \\
3.7299 E-O 1 \\
9.4632 E-01 \\
1.8539 E+00\end{array}$ & $\begin{array}{r}-1.0039 E+00 \\
-1.2016 E-01 \\
4.3667 E-C 1 \\
1.0722 E+00\end{array}$ \\
\hline 25 & $\begin{array}{r}-3.2189 E+00 \\
-8.2480 E-C 1 \\
-4.2030 E-02 \\
5.3011 E-C 1 \\
1 . C S \in 8 E+O C\end{array}$ & $\begin{array}{r}-2.1983 E+00 \\
-6.3322 E-01 \\
8.0841 E-02 \\
6.3824 E-01 \\
1.2309 E+00\end{array}$ & $\begin{array}{r}-1.67 \\
-4.64 \\
1.98 \\
7.47 \\
1.38\end{array}$ & $\begin{array}{r}-1.3216 E+00 \\
-3.1203 E-01 \\
3.1125 E-01 \\
8.5847 E-01 \\
1.5757 E+C 0\end{array}$ & $\begin{array}{r}-1.0487 E+C 0 \\
-1.7225 E-01 \\
4.2152 E-C 1 \\
5.7406 E-01 \\
1.8655 E+00\end{array}$ \\
\hline 0 & $\begin{array}{r}-3.2581 E+00 \\
-8.6873 E-C 1 \\
-9.3187 E-02 \\
4.66 C 3 E-01 \\
1.00 C 0 E+00 \\
1.8765 E+00\end{array}$ & $\begin{array}{r}-2.23 \\
-6.78 \\
2.77 \\
5.70 \\
1.11\end{array}$ & $\begin{array}{r}-1.7179 E+00 \\
-5.1074 E-C 1 \\
1.428 C E-C 1 \\
6.7428 E-01 \\
1.2511 E+00\end{array}$ & $\begin{array}{r}-1.3635 E+C 0 \\
-3.5987 E-O 1 \\
2.5342 E-01 \\
7.7956 E-C 1 \\
1.4020 E+C 0\end{array}$ & $\begin{array}{r}-1.0915 E+00 \\
-2.2106 E-01 \\
3.6082 E-C 1 \\
8.8754 E-C 1 \\
1.5502 E+00\end{array}$ \\
\hline 27 & $\begin{array}{r}-3.2959 E+C O \\
-9.1080 E-01 \\
-1.4175 E-01 \\
4.0028 E-01 \\
9.1480 E-01 \\
1.6039 E+0 C\end{array}$ & $\begin{array}{r}-2.2768 E+00 \\
-7.2149 E-01 \\
-2.2554 E-02 \\
5.0714 E-01 \\
1.0244 E+00 \\
1.887 C E+00\end{array}$ & $\begin{array}{r}-1.75 \\
-5.55 \\
9.05 \\
6.07 \\
1.14\end{array}$ & $\begin{array}{r}-1.4036 E+00 \\
-4.0548 E-01 \\
1.9905 E-01 \\
7.0781 E-01 \\
1.2702 E+00\end{array}$ & $\begin{array}{r}-1.1326 E+00 \\
-2.6807 E-01 \\
3.0393 E-C 1 \\
8.0984 E-01 \\
1.4184 E+C O\end{array}$ \\
\hline & $\begin{array}{r}-3.3317 E+00 \\
-9.5116 E-01 \\
-1.8797 E-01 \\
3.5028 E-01 \\
8.38 C O E-01 \\
1.4341 E+C O\end{array}$ & $\begin{array}{r}-2.3139 E+00 \\
-7.6283 E-01 \\
-7.0316 E-02 \\
4.4823 E-01 \\
9.4016 E-01 \\
1.617 C E+00\end{array}$ & $\begin{array}{r}-1.795 C E+00 \\
-5.9747 E-01 \\
4.1171 E-02 \\
5.4504 E-01 \\
1.0473 E+00 \\
1.897 C E+C O\end{array}$ & $\begin{array}{r}-1.4422 E+C O \\
-4.4904 E-01 \\
1.4705 E-01 \\
6.4165 E-C 1 \\
1.1619 E+00\end{array}$ & $\begin{array}{r}-1.172 C E+C O \\
-3.1350 E-C 1 \\
2.5042 E-C 1 \\
7.391 C E-C 1 \\
1.2883 E+C C\end{array}$ \\
\hline 2 & $\begin{array}{r}-3.3671 E+00 \\
-9.8954 E-01 \\
-2.3208 E-C 1 \\
2.5750 E-C 1 \\
7.6862 E-01 \\
1.3054 E+00\end{array}$ & $\begin{array}{r}-2.3496 E+00 \\
-8.0251 E-C 1 \\
-1.1580 E-01 \\
3.9317 E-01 \\
8.6443 E-01 \\
1.4489 E+00\end{array}$ & $\begin{array}{r}-1.8315 E+00 \\
-6.3812 E-C 1 \\
-5.8674 E-03 \\
4.8709 E-01 \\
9.6473 E-C 1 \\
1.6294 E+00\end{array}$ & $\begin{array}{r}-1.4794 E+C O \\
-4.9075 E-01 \\
9.8940 E-02 \\
5.8045 E-01 \\
1.069 C E+00 \\
1.9065 E+00\end{array}$ & $\begin{array}{r}-1.2100 t+00 \\
-3.5636 E-01 \\
1.9965 E-01 \\
0.7385 E-01 \\
1.1813 E+00\end{array}$ \\
\hline
\end{tabular}




\section{TABLE 9. (CONTINUED)}

$$
\begin{array}{rrrrr}
30-3.4 C 14 E+C O & -2.3842 E+00 & -1.8666 E+00 & -1.5152 E+00 & -1.2465 E+C O \\
-1.0273 E+O C & -8.4066 E-01 & -6.7716 E-01 & -5.3076 E-C 1 & -3.9740 E-01 \\
-2.7426 E-01 & -1.5922 E-01 & -5.0710 E-02 & 5.2772 E-02 & 1.5210 E-01 \\
2.4746 E-01 & 3.4441 E-01 & 4.3187 E-C 1 & 5.22 C 2 E-C 1 & 6.1428 E-01 \\
7.0391 E-C 1 & 7.9448 E-C 1 & 8.8987 E-C 1 & 9.8710 E-01 & 1.090 C E+00 \\
1.1994 E+00 & 1.3216 E+O C & 1.4631 E+C O & 1.6413 E+00 & 1.9157 E+C C
\end{array}
$$


use of ordinary regression of $z_{k}$ on $D_{k}$. If these estimates are $d_{0}$ and $d_{1}$ then $\hat{\imath}=d_{1} / d_{0}$ and

$$
\hat{a}=a_{0}(1+\hat{\Lambda})
$$

Equation 5.62 can be iterated for a better estimate. If $a_{0}$ is the true value of the parameter then $\Delta$ is a ratio of linear combinations of independent exponential random variables.

Define the statistic

$$
T=\Sigma D_{k} z_{k} / \Sigma z_{k}
$$

The statistic is a monotone function of $\wedge$ and is clearly independent of the scale parameter. Furthermore, the $T$ statistic is appealing for testing the hypothesis $\mathrm{H}_{\mathrm{O}}: \mathrm{a}=\mathrm{a}_{\mathrm{O}}$ versus $H_{A}: a a_{O}$. The distribution of the $T$ statistic under the null hypothesis is given by

$$
P(T>r)=\sum_{\substack { 1=1 \\
\begin{subarray}{c}{j=1 \\
j \neq n-1+1{ 1 = 1 \\
\begin{subarray} { c } { j = 1 \\
j \neq n - 1 + 1 } }\end{subarray}}\left(\frac{\left(D_{n-1+1}-r\right)^{n-1}}{\left.n-1+D_{j}\right)}\right)
$$

where $D_{n-m}<r<D_{n-m+1}$. Note that Equation 5.64 is 1dentical, except for the coefficients, to the distribution of the test suggested by Jackson (24) in Equation 1.20. The critical values for Equation 5.64 which we shall call Fuller's $T$, are given in Table 10. Fuller's $T$ may be written in terms of the original variates as 
TABLE 10 .

Critical values of the $T$ statistic based on a sample of size $N$ $P(T:$ Value $)=C$

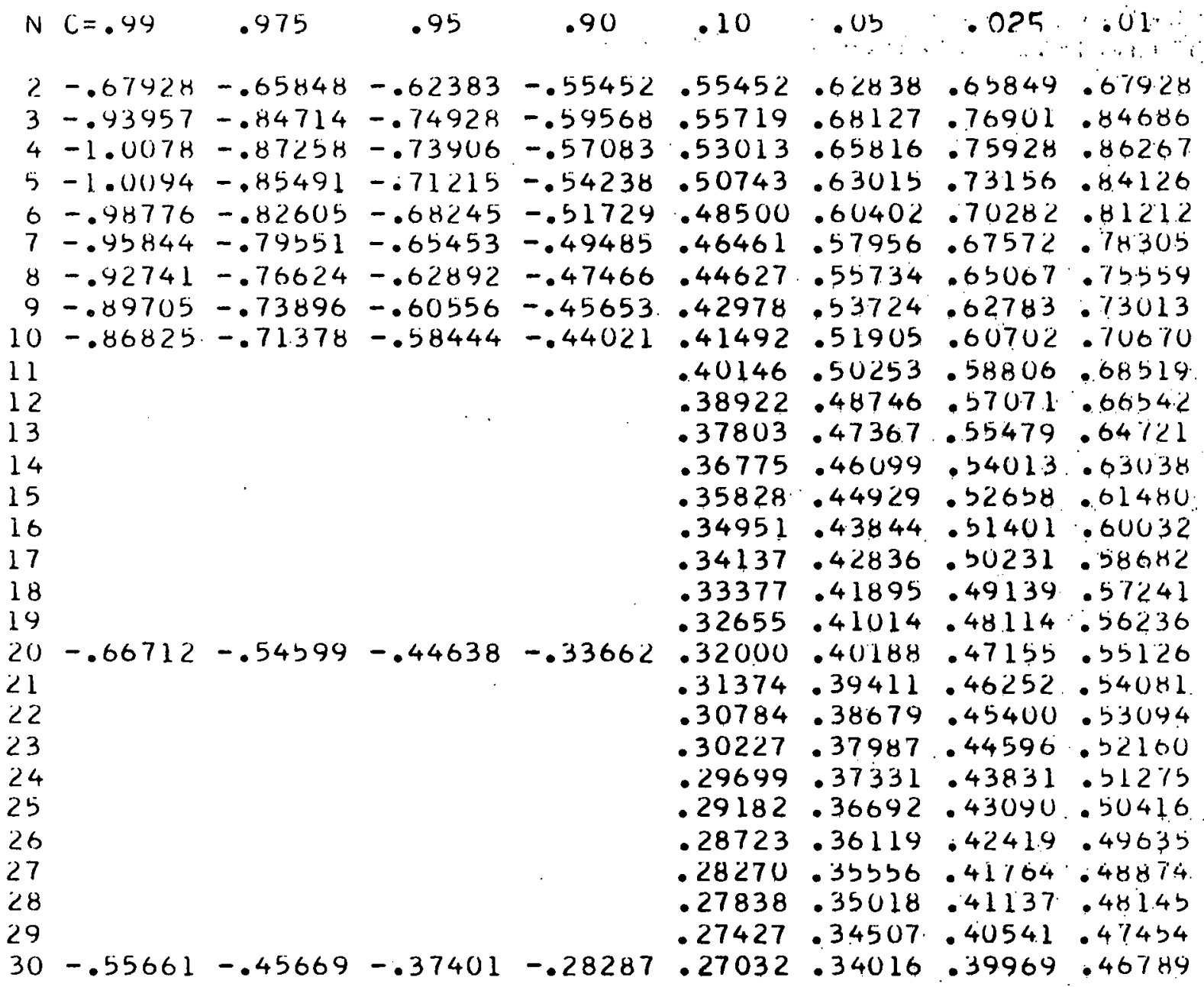




$$
T=\Sigma G_{k} Y^{1 / a_{0}}{ } / \Sigma^{1 / a_{O}}
$$

where $G_{k}=(n-k+1) D_{k}-(n-k) D_{k+1}$. Now from Equation 5.63, assuming the null hypothesis is true

$$
E\left(\Sigma D_{k} Z_{k}\right)=\stackrel{n}{\Sigma} D_{k} E\left(z_{k}\right)=0
$$

since $E\left(Z_{k}\right)$ is the same for all $k$ and the sum of the $D_{k}$ is zero.

It has been suggested by Fuller (18) that the $T$ statistic is approximately normal with varlance $\Sigma D_{k}{ }^{2} / n^{2}$. For $n=30$, the sum of squares is 44.16 and the square root is 6.65 . It is known that if $X$ is distributed $N\left(0, a^{2}\right)$ then $\frac{1}{a} X$ is distributed as $\mathrm{N}(0,1)$ : Hence the critical values of Fuller's $T$ for $n=30$ multiplied by $\sqrt{n^{2} / \Sigma D_{k}^{2}}=4.51$, should be approximately equal to the corresponding critical values of the normal distribution. The results in Table 11 suggest that the use of a normal approximation for small sample sizes would be somewhat inaccurate. Jackson (24) suggests that for his similar test, given in Equation 1.21, the approach to normality is rather slow, it appears that the normal approximation to Fuller's $T$ would be sat1sfactory for large samples, especialiy for two talled tests.

White (47) and others suggest transformed estimators based on the extreme value distribution as discussed in Chapter $I$. The transformation is the natural logarithm 
Table 11.

Comparison of $\mathrm{T}$ to normal

for $n=30$

\begin{tabular}{lllllllll}
\hline Level & .99 & .975 & .95 & .90 & .10 & .05 & .025 & .01 \\
\hline Normal & -2.33 & -1.96 & -1.64 & -1.28 & 1.28 & 1.64 & 1.96 & 2.33 \\
T. & -2.51 & -2.06 & -1.68 & -1.28 & 1.22 & 1.54 & 1.80 & 2.12 \\
\hline
\end{tabular}


of the observations. That is if $\mathrm{X}$ is a Weibull random var1able then if $Y=\log X$ the distribution of $Y$ is

$$
\begin{aligned}
F(y) & =P(Y<y)=P(\log X<y) \\
& =P\left(X<e^{y}\right) \\
& =1-\exp \left(-\left(e^{y / c}\right)^{1 / a}\right) \\
& =1-\exp \left(-\exp \left(\frac{1}{a}:(y-\log c)\right)\right)
\end{aligned}
$$

Let $A=\log c$ and $B=a$, then the reduced random variable $U=(Y-A) / B$ has distribution

$$
F(u)=P(Y<A+B u)=1-e^{-e^{X}}
$$

The estimators are given by

$$
\hat{A}=\Gamma A_{1} Y_{(1)}, \hat{B}=\Gamma B_{1} Y(1)
$$

where the coefficients $A_{1}$ and $B_{1}$ are generated using the least square procedure of Lloyd (27) on the reduced var1ables. Hence the estimates are unblased and have minimum variance in the class of unbiased estimates. White then Gives tables of the expected values, variances, and covariances of the reduced log Welbull variate and develops and tabulates the coefficients. Finaliy White computes the variances and covariances of the estimates.

As a test of the hypothesis $\mathrm{H}_{O}: a=a_{O}$ versus $\mathrm{H}_{A}: a \neq a_{O}$, we can use the statistic 


$$
W=\frac{\hat{B}-a_{0}}{\sqrt{V(\hat{B})}} .
$$

Unfortunately little is known about the distribution of the estimators and Monte Carlo techniques must be used to get the critical points of the test.

Conslder the likelihood ratio test of $\mathrm{H}_{0}: \mathrm{a}=\mathrm{a}_{0}$ versus $\mathrm{H}_{\mathrm{A}}: \mathrm{a}=\mathrm{a}_{1}$. Recall the density functions

$$
f(y ; a, c)=\frac{c^{-1 / a}}{a} y^{\frac{1}{a}-1} \exp \left(-(y / c)^{1 / a}\right) .
$$

The likelihood function is

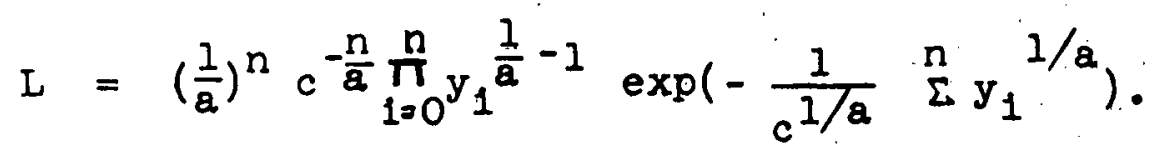

Hence the likelihood ratio is

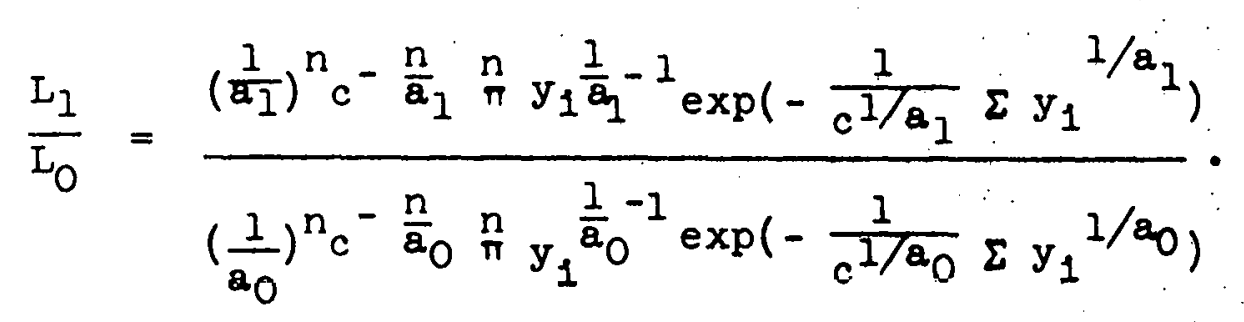

From Equation 1.12 the MIE estimate for the scale parameter is

$$
\hat{c}=\left(\frac{l}{n} \Sigma y_{1}^{1 / \hat{a}}\right)^{\hat{u}}
$$

Substituting the above into Equation 5.43 the likelihood ratio is 
$\frac{L_{1}}{L_{0}}=\left(\frac{a_{0}}{a_{1}}\right)^{n}\left(\frac{\frac{l}{n} \sum y_{1}^{1 / a_{1}}}{\frac{1}{n} \sum y_{1}^{1 / a_{0}}}\right)^{-n} \frac{n}{n y_{1}}{ }^{1 / a_{1}}-1 / a_{0} \frac{e^{-1}}{e^{-1} \cdot(5.73)}$

Therefore the test is to accept $\mathrm{H}_{\mathrm{O}}$ if

$$
R=\left(\frac{r y_{1}^{1 / a_{0}}}{r y_{1}{ }^{l / a_{1}}}\right)^{n}\left(n y_{1}\right)^{1 / a_{1}-1 / a_{0}}>k \text {. }
$$

The distribution of the test is not known so that Monte Carlo methods are used to determine the critical points.

The three tests given in Equation 5.63, 5.70, and 5.74 were compared using Monte Carlo methods. The null hypothesis was that the shape parameter, a, was one, that 1s, that the underlying distribution was exponential. The alternatives investigated were Weitull with shape parameters $.8,1.2,2.0$, and 3.0. The Monte Carlo study consisted in generating 6,000 samples of size 5, 10, and 20. The three null test statistics were computed for each sample using the exponential as the underlying distribution and then ordered in increasing magnitude. Then the critical values under the null hypothesis were found by identifying the desired percentage points. The test was then calculated using as the underlyinf distribution a Weibull with shape parameter equal to that of the alternative hypothesis. These statistics were then placed in increasing order. The fraction of the sample exceeding the previously determined critical value gave the empirical 
power of the tests. For the alternative shape parameter larger than one the critical point for a one sided test with alternative hypothesis shape parameter greater than one was used. Conversely the power of the one sided test for shape parameter less than one was compared for the Weibull with shape parameter of .8. The results appear in Table 12. The likelihood test proved to be the most powerful since the likelihood ratio test is always a most powerful test. Furthermore since the underlying distribution of the alternative was always that of the alternative hypothesis the simple versus simple likelihood ratio was in its most ideal situation. Had wo used a test statistic based on anderlying distribution with some other shape parameter than that given in the alternative hypothesis we would expect the power to be reduced. The power of the log Weibull based test and Fuller's $T$ were approximately the same. As the underlying distribution deperts from the exponential the power of the three tests becomes more uniform. The power also increases rapidiy as sample size increases. 
Táble 12.

Powers of tests based on a sample of size $\mathrm{N}$ of the hypothesis that the distribution is exponential where the alternative distribution is Weibuli with shape parameter a

\begin{tabular}{|c|c|c|c|c|c|c|c|c|c|c|c|c|}
\hline \multicolumn{4}{|c|}{ Size of test .10} & \multicolumn{3}{|c|}{.05} & \multicolumn{3}{|c|}{.025} & \multicolumn{3}{|c|}{.01} \\
\hline$N=5$ & $T^{\perp}$ & $W$ & $R$ & $T$ & $w$ & $k$ & $T$ & $w$ & $R$ & $T$ & $w$ & $R$ \\
\hline $\begin{array}{l}.8 \\
1.2 \\
2.0 \\
3.0 \\
N=10\end{array}$ & $\begin{array}{r}.193 \\
.218 \\
.6110 \\
.822\end{array}$ & $\begin{array}{l}.197 \\
.226 \\
.678 \\
.893\end{array}$ & $\begin{array}{l}.208 \\
.272 \\
.682 \\
.897\end{array}$ & $\begin{array}{r}.115 \\
.128 \\
.492 \\
.740\end{array}$ & $\begin{array}{r}.119 \\
.142 \\
.524 \\
.749\end{array}$ & & & $\begin{array}{l}.065 \\
.083 \\
.405 \\
.684\end{array}$ & $\begin{array}{l}089 \\
090 \\
420 \\
690\end{array}$ & & $\begin{array}{r}.025 \\
.046 \\
.274 \\
.531\end{array}$ & $\begin{array}{r}.051 \\
.047 \\
.336 \\
.535\end{array}$ \\
\hline $\begin{array}{l}=.8 \\
1.2 \\
2.0 \\
3.0 \\
N=20\end{array}$ & $\begin{array}{r}.282 \\
.282 \\
.845 \\
.976\end{array}$ & $\begin{array}{l}.282 \\
.288 \\
.884 \\
.981\end{array}$ & $\begin{array}{l}.299 \\
.308 \\
.904 \\
.984\end{array}$ & $\begin{array}{r}.176 \\
.173 \\
.753 \\
.959\end{array}$ & $\begin{array}{r}.180 \\
.183 \\
.813 \\
.962\end{array}$ & $\begin{array}{l}.214 \\
.207 \\
.821 \\
.972\end{array}$ & & $\begin{array}{l}.093 \\
.108 \\
.726 \\
.952\end{array}$ & & & $\begin{array}{r}.033 \\
.062 \\
.032 \\
.011\end{array}$ & $\begin{array}{l}.061 \\
.086 \\
.041 \\
.926\end{array}$ \\
\hline $\begin{array}{l}=.8 \\
1.2 \\
2.0 \\
3.0\end{array}$ & $\begin{array}{l}.421 \\
\because 407 \\
.980 \\
1.00\end{array}$ & $\begin{array}{r}.441 \\
.412 \\
.987 \\
1.00\end{array}$ & $\begin{array}{l}.512 \\
.548 \\
.994 \\
1.00\end{array}$ & $\begin{array}{r}.281 \\
.275 \\
.961 \\
.999\end{array}$ & $\begin{array}{r}.286 \\
.285 \\
.977 \\
1.00\end{array}$ & $\begin{array}{l}.321 \\
.338 \\
.981 \\
1.00\end{array}$ & $\begin{array}{r}.204 \\
.185 \\
.934 \\
.998\end{array}$ & $\begin{array}{r}.205 \\
.187 \\
.943 \\
1.00\end{array}$ & $\begin{array}{r}.241 \\
.231 \\
.960 \\
1.00\end{array}$ & $\begin{array}{l}.094 \\
.112 \\
.890 \\
.996\end{array}$ & $\begin{array}{r}.090 \\
.114 \\
.921 \\
.999\end{array}$ & $\begin{array}{l}.140 \\
.160 \\
.930 \\
.999\end{array}$ \\
\hline
\end{tabular}

$I_{T}$ is Fuller's T statistic, W is White's log Welbull statistic, and $R$ is the likelihood ratio test. $R$ is simple versus simple where the alternative hypothesis is the alternative used to get power. 
CHAPTER VI. MOMENTS OF SYMMETRIC DISTRIBUTIONS FROM THEIR HALF DISTRIBUTIONS

Suppose $Y$ is a random variable with density function $f(y) \geqslant 0$ for $>0$ and $f(y)=0$ otherw1se. Let $z$ be the random variable with density function $g(z)=(1 / 2) f(z)$ if $z>0$ and $g(z)=(1 / 2) f(-z)$ if $z<0$. Consider the conditional density of $z$ given that $z>0$. We know that

$$
\begin{aligned}
g(z \mid z>0) & =g(z, z>0) / P(z>0) \\
& =g(z, z>0) /(1 / 2) \\
& =2 g(z, z>0) \\
& =2(1 / 2) f(z) \\
g(z \mid z>0) & =f(z) .
\end{aligned}
$$

Suppose a sample of size $n$ is drawn from $g(z)$. Let $J$ be the random variable equal to the number of observations greater than zero. Obviously $J$ is a binomial varlate with parameter $p=0.5$. Then the conditional distribution of the $J=J$ positive values of a sample of size $n$ from the symmetric density $g(z)$ given that $J=g$ is precisely that of a sample of size $f$ drawn from the half distribution $f(z)$. Similarly, the conditional distribution of the $n-j$ negative observations given that $J=f$ is precisely that of a sample 
of. size $n-j$ drawn from the half distribution $f(-z)$. If the $Z_{(1)}$ are the ordered random variables, we are able to write for fixed $k, 0<k<j$

$$
g\left(z_{(n-k)} \mid J=j\right)=f_{X(j-k)}\left(z_{(j-k)}\right)
$$

where $f(z(j-k))$ is the distribution of the $(j-k)$-th order statistic from a sample of $f$ observations. Since there are $n-j$ observations less than zero we use the same argument for $k \geqslant j$ to write

$$
\left.\left.g(z(n-k) \mid J=j)=f_{(n-j+1)}\right)^{(-z}(k-j+1)\right)
$$

where $f(-z(k-j+1))$ is the distribution of the $(k-j+l)-s t$ order stat1st1c from a sample of size $n-j$. Then for fixed $k$

$$
\begin{aligned}
E\left(Z_{(n-k)} \mid J=j\right) & =E\left(Y_{(j-k)} \text { of } j\right) \text { if } 0<k<j \\
& =E\left(-Y_{(k-j+1)} \text { of } n-j\right) \text { if } k \geqslant j
\end{aligned}
$$

where the expected values on the rlght-hand side are based on samples of size $J$ and $n-J$ respectively. Since $J$ is a random $E\left(Z_{(n-k)}\right)=E_{j}\left(E\left(Z_{(n-k)} \mid J=j\right)\right)$. The expected value, $\frac{E}{J}, 1 s$ the sum over all the possible values of $J$ multiplied by $P(J=J)$. Hence for any $k, 0 \leqslant k<n-1$

$$
\begin{aligned}
E(Z(n-k)) & =\sum_{j=0}^{k} P(n, j) E(-Y(k-j+1) \text { of } n-j) \\
& +\sum_{j=k+1}^{n} P(n, j) E(Y(j-k) \text { of } j)
\end{aligned}
$$


where $P(n, j)=\left(\begin{array}{l}n \\ j\end{array}\right)(1 / 2)^{n}$.

Through a similar argument we are able to find the expected values of the crossproducts of order statistics of the symmetric distribution as

$$
\begin{aligned}
E(Z(1) Z(k)) & =\sum_{j=0}^{i-1} P(n, j) E(Y(1-j) Y(k-j) \text { of } n-j) \quad(6.7) \\
& +\sum_{j=1}^{k-1} P(n, j) E(-Y(j-1+1) \text { of } j) E(Y(k-j) \text { of } n-j) \\
& +\sum_{j=k}^{n} P(n, j) E((-Y(j-k+1))(-Y(j-1+1) \text { of } j)
\end{aligned}
$$

where $i<k$.

\section{A. Moments of the Double Weibull}

In Chapter II the moments for the order statistics of the Weibuli distribution with integer shape parameter were derived from the moments of a transformed random variable $x$ where $X=Y^{1 / a}$ and $Y$ is the Welbull variate. The recursive relation defining the moments is given by

$$
E\left(X_{(k)}^{m}\right)=m: \sum_{1=0}^{m} E\left(x_{(k-1)}^{1}\right)\left(\frac{1}{n-k+1}\right)^{m-1} \text {. }
$$

From the transformation we have the $q$-th moment for a Weibull with scale parameter $c$ and shape parameter $a$,

$$
E(Y(k))=c^{q} E\left(X_{(k)}^{q q}\right)
$$

The only restriction is that aq be a positive integer. If 
we write the density function of $\mathrm{Y}$ as

$$
\begin{aligned}
f(y ; a, b) & =\frac{1}{a b} y^{\frac{1}{a}-1} \exp \left(-y^{1 / a} / b\right) \quad y>0, a>0, b>0 \\
& =0 \text { otherwise } \quad(6.10)
\end{aligned}
$$

then the symmetric density related to the above density is given by

$$
\begin{aligned}
f(z ; a, b)= & \frac{1}{2 a b}|z|^{\frac{1}{a}-1} \exp \left(-|z|^{1 / a / b}\right)-m<z<\infty \\
& a>0, b>0
\end{aligned}
$$

We seek the moments of $Z$ as a function of the moments of $Y$. Hence from Equations 6.6 and 6.9 we have

$$
\begin{aligned}
E(z \underset{(n-k)}{m}) & =\sum_{j=0}^{k} P(n, j) E\left((-X(k-j+1))^{m} \text { of } n-j\right) \\
& +\sum_{j=k+1}^{n} P(n, j) E(X(j-k) \text { of } j) .
\end{aligned}
$$

$z^{m}$ is the $q$-th moment of a double welbull with shape parameter a. such that $m=q a$. The values derived from Equation 6.12 may be multiplied by $b^{\text {aq }}$ to generate the moments of welbuils with nonunit scale parameters. Figure 1, presented here, from (29) is for the double Weibull and we find the value for the $q$-th moment of a double welbull with shape parameter a in the aq-th column of the table. For example, if $Y(4)$ is the fourth order statistic in a sample of size five with shape parameter two then $E\left(Y_{(4)}\right)=0.830695$ and $E\left(Y_{(4)}^{2}\right)=$ 
MCMENTS $U=$ YHE XTH CROER STATISTIC

FRCY THE OCUSLE EXPONENTIAL

- OISTRIBUTICN

$E\{(x(K) \neq+1)\}$

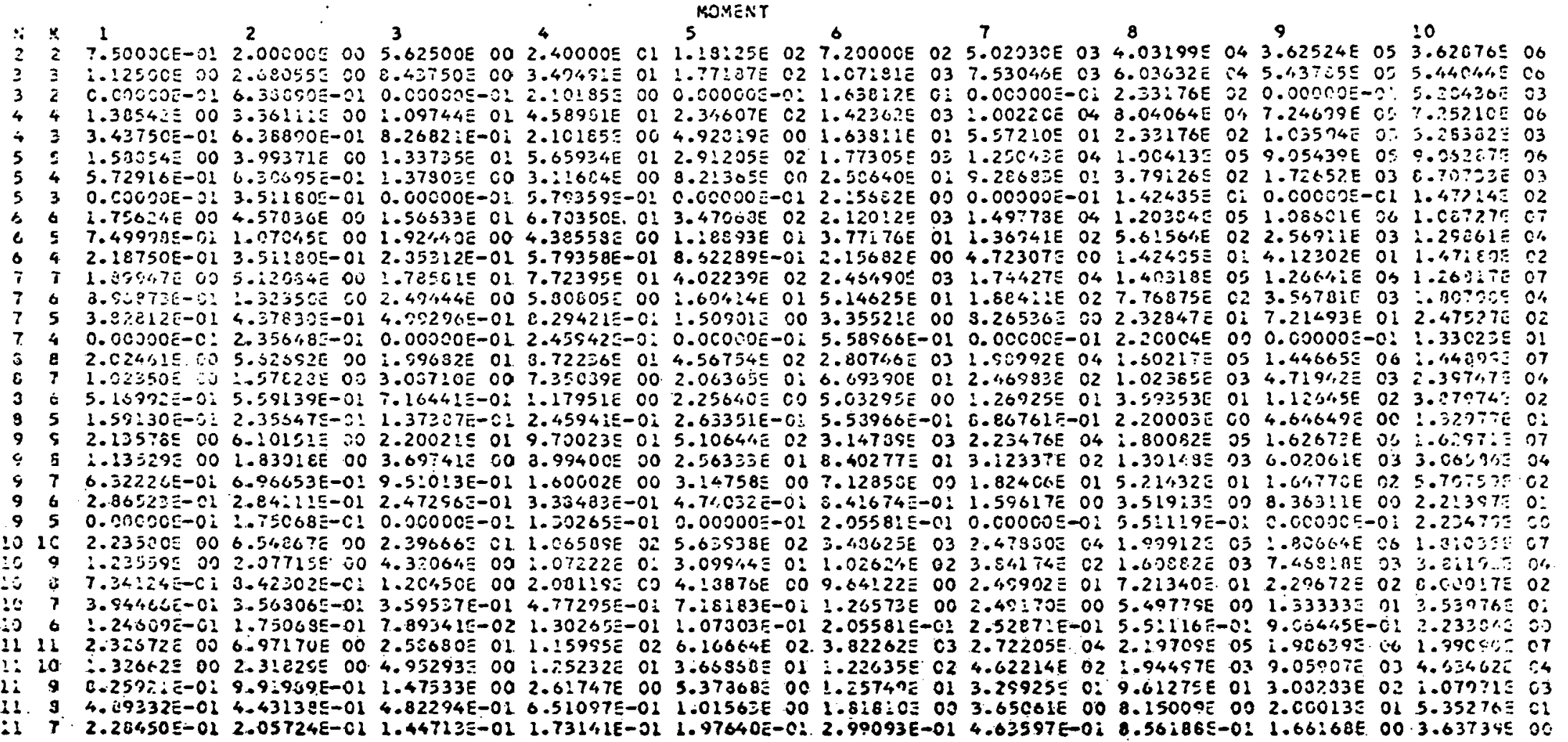

P1gure 1. Moments of the $K-t h$ order statistic from the double exponential distribution. 
:1

$\begin{array}{ll}12 & 0 \\ 12 & 2 \\ 12 & \vdots \\ 12 & 9\end{array}$

$1: 29$

$\begin{array}{ll}2 & 3 \\ 2 & 9\end{array}$

is

政

30

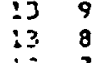

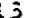

$\because \because 4$ is

i. 12

$\because ":=$

1410

is 8

$\begin{array}{ll}15 & : 5 \\ 15 & 14\end{array}$

is 15

$15 \div 2$

15 iो

$\begin{array}{ll}15 & 20 \\ 15 & 5\end{array}$

is

is $i 5$

is 14

is 13

: 6 il

io $: c$

$\div 69$

$\vdots 16$

$: 7: 5$

:7 is

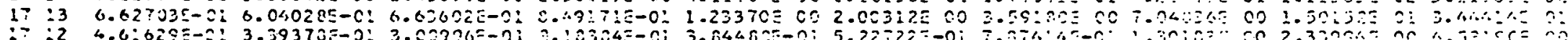

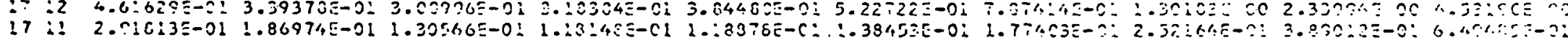

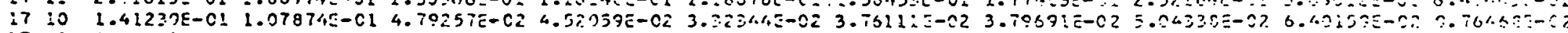

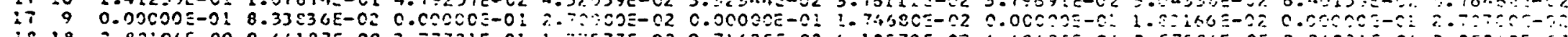

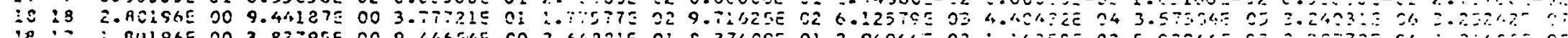

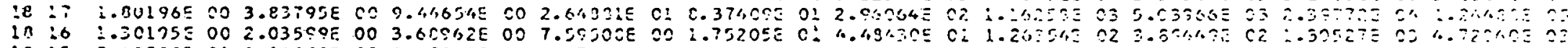

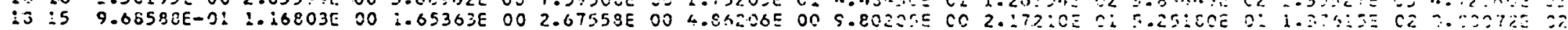

\section{Pigure 1. (Continued)}


$\therefore 14$

$\because: 2$

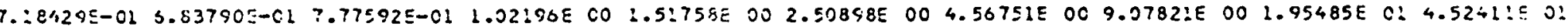
(173:-31 作

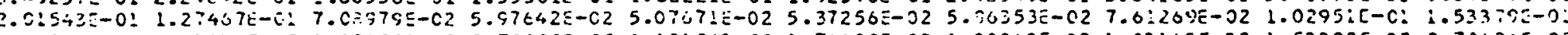

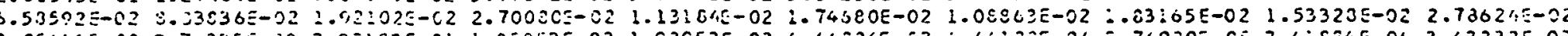

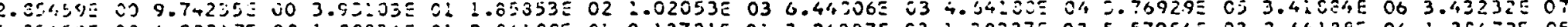
C. (.)

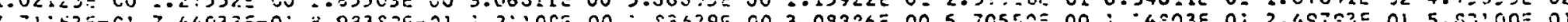
7.

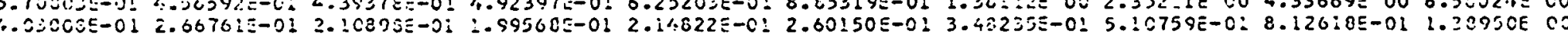

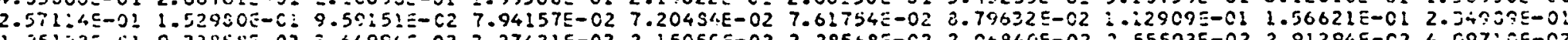

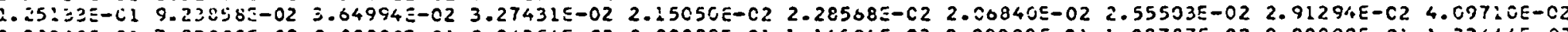

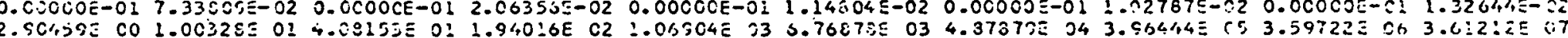

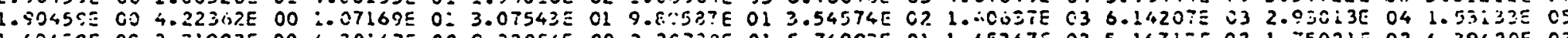

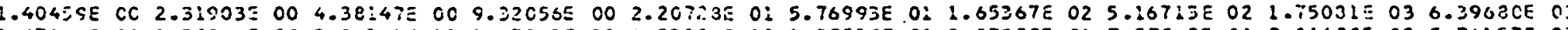

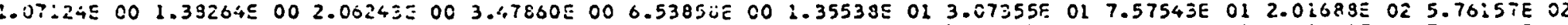

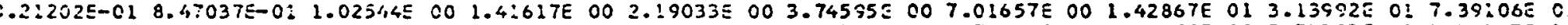

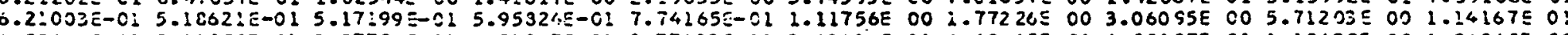

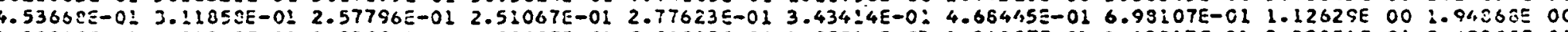

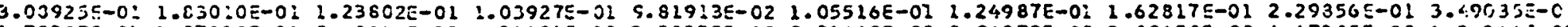

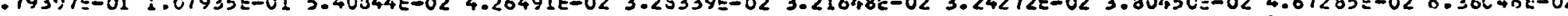
$\begin{array}{llll}0 & \end{array}$

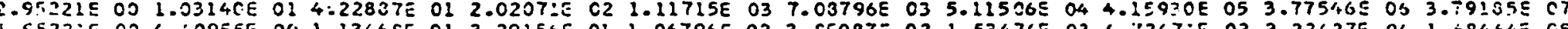

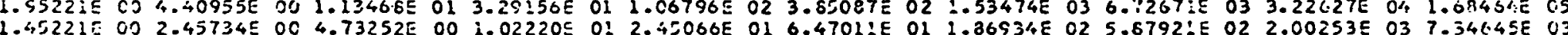

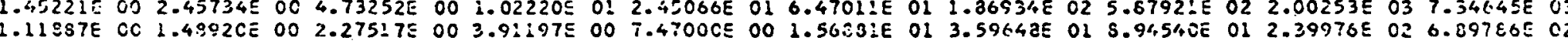

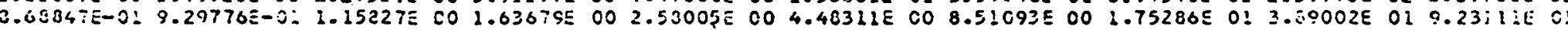

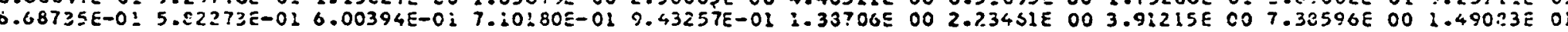

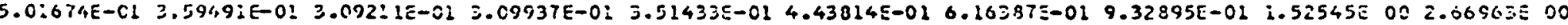

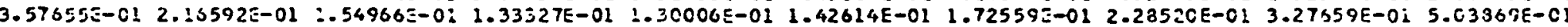

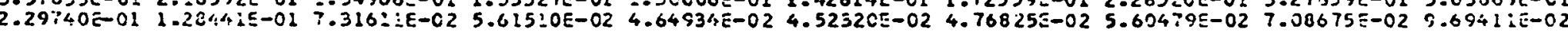

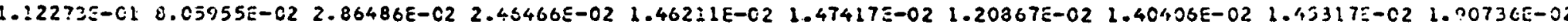

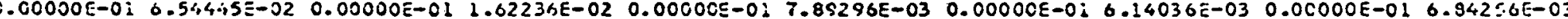

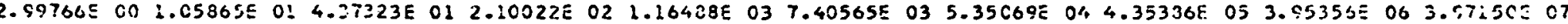

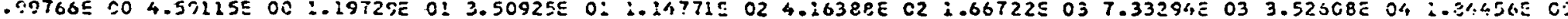

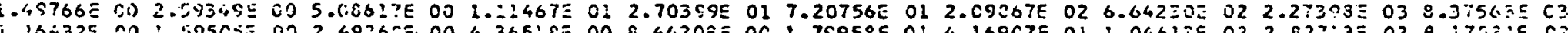

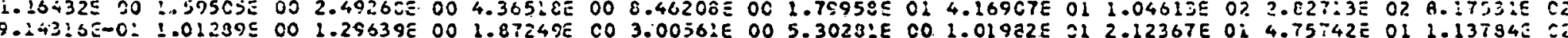

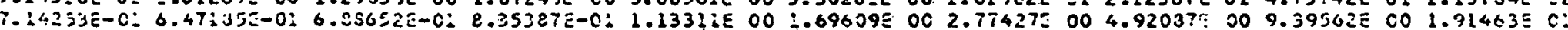

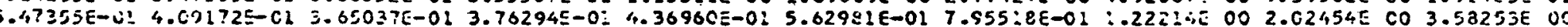

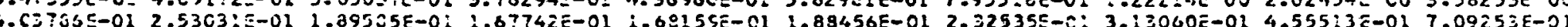
(1)

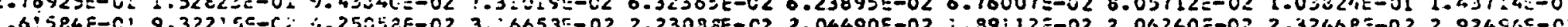
10.

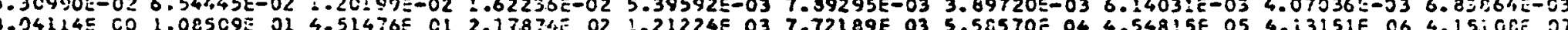

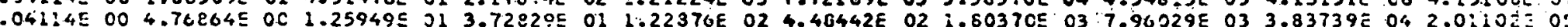

F1gure 1. (Cantinued) 


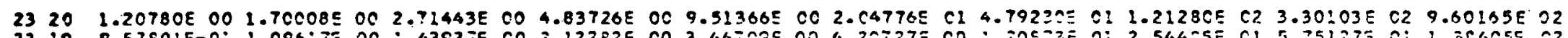

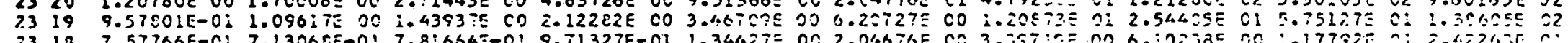

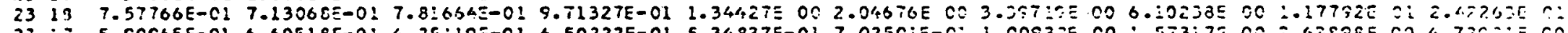

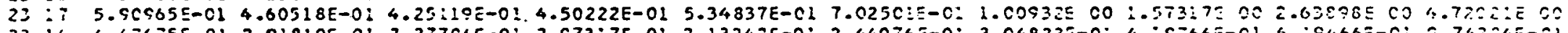

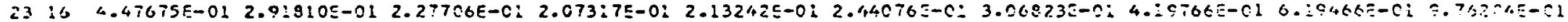

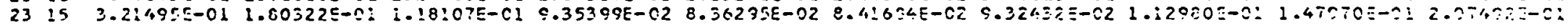

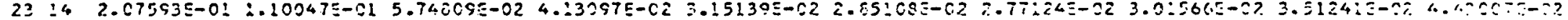

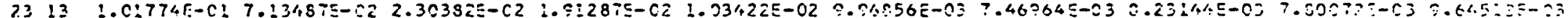

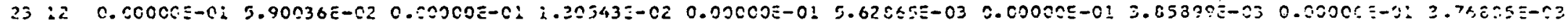

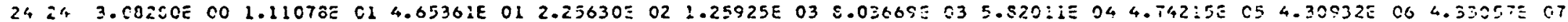

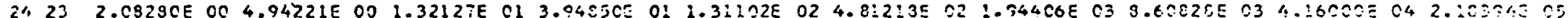

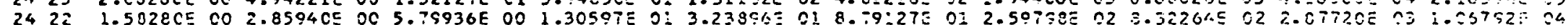

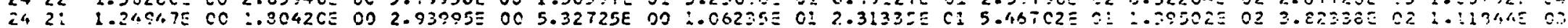

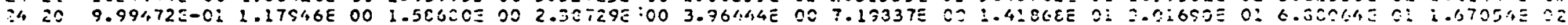

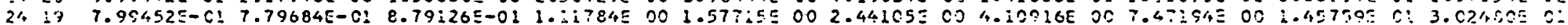

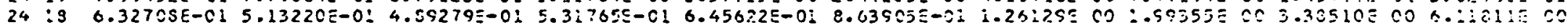

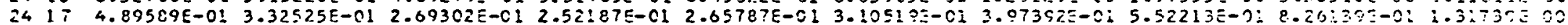

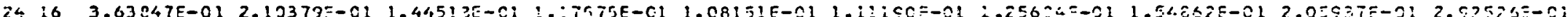

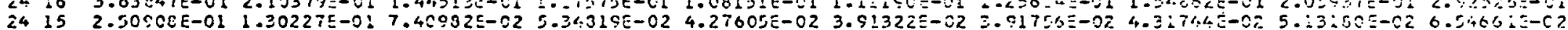

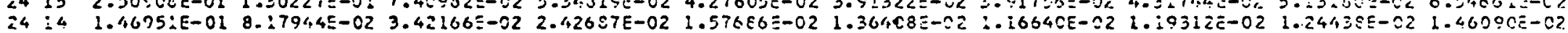

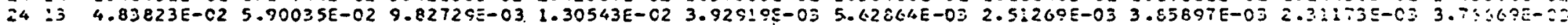

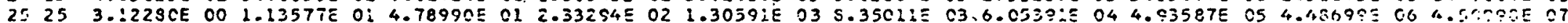

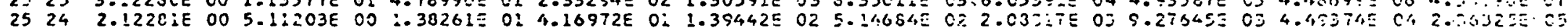

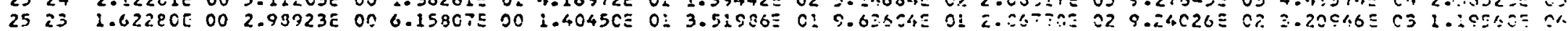

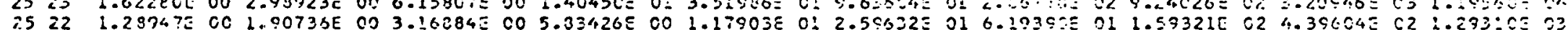

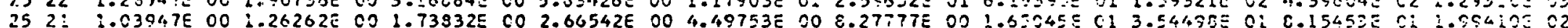

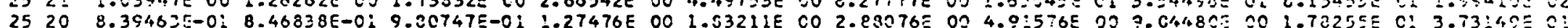

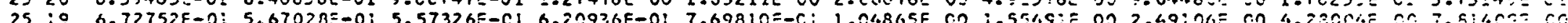

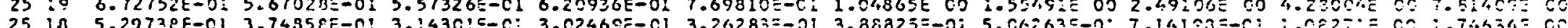

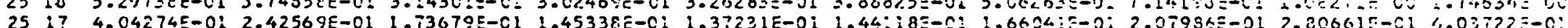
25 in $2.51976 E-01$ l. $53152 E-01$ 9.

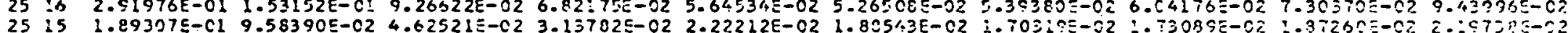

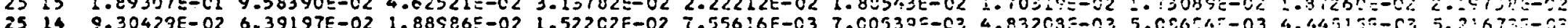

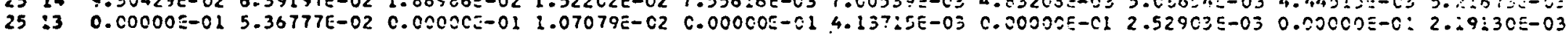

Figure 1. (Continued) 
3.11684. For values not in the table use the relation $E(Y(j)=-E(Y(n-j))$.

From Equations 6.7 and 6.9 the moments of the crossproducts are

$$
\begin{aligned}
& E\left(\left(Z(1)^{Z}(k)\right)^{m}\right)=\sum_{j=0}^{1=1} P(n, j) E\left(\left(x(1-j)^{X}(k-j)^{m} \text { of } n-j\right)\right. \\
& +\sum_{j-1}^{k-1} P(n, j) E\left((-x(j-1+1))^{m} \text { of } j\right) E((x, j-k) \text { of } n-j) \\
& +\sum_{j=k}^{n} P(n, j) E\left(((-x(j-k+1))(-x(j-1+1)))^{m} \text { of } j\right)
\end{aligned}
$$

where $1<j$. For the double Welbull with shape parameter a, the $q$-th moment of the crossproducts is found in the aq-th column of Table 13. For example $E\left(z(1)^{Z}(2)\right.$ ) of a double Weibull with shape parameter of three in a sample of flve is 77.428 .

Our results were compared to those of Sarhan (36) for the double exponential of samples up to size flve and agreed on the fifth digit.

The combinatorial forms of Equations 6.6 and 6.7 are not dependent on the distribution. Thus we are able to generate the moments of the order statistics of any symmetric distribution from the moments of the related half distribution. For example, we are able to get the moments of the lsoceles triangular from the right triangular distribution, the uniform $(-a, a)$ from the uniform $(0, a)$, and the normal from the 
TABLE 13.

Expected crossproducts of the 1 -th and $j^{-t h}$ order statistics of the double Welbull with shape parameter a based on a

\begin{tabular}{|c|c|c|c|c|c|c|c|}
\hline & $\begin{array}{l}1 \\
1\end{array}$ & 2 & $\begin{array}{c}1 \\
0.0000 E+00\end{array}$ & 4. $20000 E+00$ & $\begin{array}{c}3 \\
0.0000 E+00\end{array}$ & $\begin{array}{c}4 \\
5.7600 E+02\end{array}$ & $\begin{array}{c}5 \\
1.5625 E-02\end{array}$ \\
\hline & $\begin{array}{l}1 \\
1 \\
2\end{array}$ & $\begin{array}{l}2 \\
3 \\
3\end{array}$ & $\begin{array}{r}4.7222 E-01 \\
-9.4444 E-01 \\
4.7222 E-01\end{array}$ & $\begin{array}{l}3.1574 E+00 \\
5.6852 E+00 \\
3.1574 E+00\end{array}$ & $\begin{array}{r}2.4210 E+01 \\
-4.8420 E+01 \\
2.4210 E+01\end{array}$ & $\begin{array}{l}4.3591 E+02 \\
8.5618 E+02 \\
4.3591 E+02\end{array}$ & $\begin{array}{r}1.050 \\
-2.100 \\
1.050\end{array}$ \\
\hline 4 & $\begin{array}{l}1 \\
1 \\
1 \\
2 \\
2 \\
3\end{array}$ & $\begin{array}{l}2 \\
3 \\
4 \\
3 \\
4 \\
4\end{array}$ & $\begin{array}{r}9.4444 E-01 \\
-2.0139 E-01 \\
-1.6875 E+00 \\
2.0139 E-01 \\
-2.0139 E-01 \\
9.4444 E-01\end{array}$ & $\begin{array}{l}5.0370 E+00 \\
1.9259 E+00 \\
9.4444 E+00 \\
6.2963 E-01 \\
1.9259 E+00 \\
5.0370 E+00\end{array}$ & $\begin{array}{r}4.8420 E+01 \\
-1.9176 E+00 \\
-9.4922 E+01 \\
1.9176 E+00 \\
-1.9176 E+00 \\
4.8420 E+01\end{array}$ & $\begin{array}{l}E+02 \\
E+01 \\
E+03 \\
E+01 \\
E+01 \\
E+02\end{array}$ & $\begin{array}{l}E+04 \\
E+02 \\
E+04 \\
E+02 \\
E+02 \\
E+04\end{array}$ \\
\hline 5 & $\begin{array}{l}1 \\
1 \\
1 \\
1 \\
2 \\
2 \\
2 \\
3 \\
3\end{array}$ & $\begin{array}{l}2 \\
3 \\
4 \\
5 \\
3 \\
4 \\
5 \\
4 \\
5 \\
5\end{array}$ & $\begin{array}{r}1.3927 E+00 \\
2.6403 E-01 \\
-7.1062 E-01 \\
-2.3387 E+00 \\
2.8017 E-01 \\
-1.1375 E-01 \\
-7.1062 E-01 \\
2.8017 E-01 \\
2.6403 E-01 \\
1.3927 E+00\end{array}$ & $\begin{array}{l}7.5396 E+00 \\
1.9088 E+00 \\
2.6471 E+00 \\
1.3976 E+01 \\
6.5757 E-01 \\
5.1787 E-01 \\
2.6471 E+00 \\
6.5757 E-01 \\
1.9088 E+00 \\
7.5396 E+00\end{array}$ & $\begin{array}{r}7.74 \\
7.32 \\
-1.19 \\
-1.50 \\
2.48 \\
-3.49 \\
-1.19 \\
2.48 \\
7.32 \\
7.74\end{array}$ & $\begin{array}{l}1.3330 E+03 \\
8.9736 E+01 \\
1.2044 E+02 \\
2.6316 E+03 \\
1.8083 E+01 \\
5.8829 E+00 \\
1.2044 E+02 \\
1.8083 E+01 \\
8.9736 E+01 \\
1.3330 E+03\end{array}$ & $\begin{array}{l}32 E+04 \\
48 E+03 \\
0 E+03 \\
30 E+04 \\
5 E+02 \\
9 E+00 \\
50 E+03 \\
5 E+02 \\
+8 E+03 \\
32 E+04\end{array}$ \\
\hline $\begin{array}{l}6 \\
6\end{array}$ & $\begin{array}{l}1 \\
1 \\
1 \\
1 \\
1 \\
2 \\
2 \\
2 \\
2 \\
3 \\
3 \\
3 \\
4 \\
4 \\
5\end{array}$ & $\begin{array}{l}2 \\
3 \\
4 \\
5 \\
6 \\
3 \\
4 \\
5 \\
6 \\
4 \\
5 \\
6 \\
5 \\
6 \\
6\end{array}$ & $\begin{array}{r}1.8169 E+00 \\
6.5102 E-01 \\
-1.9828 E-01 \\
-1.1569 E+00 \\
-2.9297 E+00 \\
4.3738 E-01 \\
2.7656 E-02 \\
-3.9656 E-01 \\
-1.1569 E+00 \\
1.7062 E-01 \\
2.7656 E-02 \\
-1.9828 E-01 \\
4.3738 E-01 \\
6.5102 E-01 \\
1.8169 E+00\end{array}$ & $\begin{array}{l}7 E+01 \\
3 E+00 \\
5 E+00 \\
0 E+00 \\
5 E+01 \\
7 E+00 \\
5 E-01 \\
9 E-01 \\
0 E+00 \\
5 E-01 \\
5 E-01 \\
5 E+00 \\
7 E+00 \\
3 E+00 \\
7 E+01\end{array}$ & $\begin{array}{r}1.1 \\
1.51 \\
-9.62 \\
-2.26 \\
-2.14 \\
4.4 \\
4.37 \\
-1.92 \\
-2.26 \\
5.24 \\
4.37 \\
-9.62 \\
4.47 \\
1.51 \\
1.11\end{array}$ & $\begin{array}{l}1.9526 E+03 \\
1.5440 E+02 \\
3.5731 E+01 \\
2.1367 E+02 \\
3.8405 E+03 \\
3.3432 E+01 \\
3.7624 E+00 \\
1.0124 E+01 \\
2.1367 E+02 \\
2.2200 E+00 \\
3.7624 E+00 \\
3.5731 E+01 \\
3.3432 E+01 \\
1.5440 E+02 \\
1.9526 E+03\end{array}$ & $\begin{array}{r}5.1232 E+04 \\
2.1846 E+03 \\
-3.1863 E+01 \\
-2.8895 E+03 \\
-1.0174 E+05 \\
3.7493 E+02 \\
1.8311 E+01 \\
-6.3717 E+01 \\
-2.8895 E+03 \\
1.3540 E+01 \\
1.8311 E+01 \\
-3.1863 E+01 \\
3.7493 E+02 \\
2.1846 E+03 \\
5.1232 E+04\end{array}$ \\
\hline & & 2 & $1 E+00$ & +01 & .02 & $.6725 E+03$ & $E+04$ \\
\hline
\end{tabular}


TABLE 13. (CONTINUED)

$\begin{array}{lllr}7 & 1 & 3 & 1.0019 E+00 \\ 7 & 1 & 4 & 1.8242 E-01 \\ 7 & 1 & 5 & -5.8033 E-01 \\ 7 & 1 & 6 & -1.5678 E+00 \\ 7 & 1 & 7 & -3.4744 E+00 \\ 7 & 2 & 3 & 6.2082 E-01 \\ 7 & 2 & 4 & 1.8483 E-01 \\ 7 & 2 & 5 & -1.9424 E-01 \\ 7 & 2 & 6 & -6.6632 E-01 \\ 7 & 2 & 7 & -1.5678 E+00 \\ 7 & 3 & 4 & 1.9676 E-01 \\ 7 & 3 & 5 & 1.3785 E-02 \\ 7 & 3 & 6 & -1.9424 E-01 \\ 7 & 3 & 7 & -5.8033 E-01 \\ 7 & 4 & 5 & 1.9676 E-01 \\ 7 & 4 & 6 & 1.8483 E-01 \\ 7 & 4 & 7 & 1.8242 E-01 \\ 7 & 5 & 6 & 6.2082 E-01 \\ 7 & 5 & 7 & 1.0019 E+00 \\ 7 & 6 & 7 & 2.2191 E+00 \\ & & & \\ 8 & 1 & 2 & 2.6013 E+00 \\ 8 & 1 & 3 & 1.3307 E+00 \\ 8 & 1 & 4 & 5.0656 E-01 \\ 8 & 1 & 5 & -1.8180 E-01 \\ 8 & 1 & 6 & -9.2315 E-01 \\ 8 & 1 & 7 & -1.9536 E+00 \\ 8 & 1 & 8 & -3.9814 E+00 \\ 8 & 2 & 3 & 8.1437 E-01 \\ 8 & 2 & 4 & 3.4825 E-01 \\ 8 & 2 & 5 & -2.1475 E-02 \\ 8 & 2 & 6 & -4.0464 E-01 \\ 8 & 2 & 7 & -9.2801 E-01 \\ 8 & 2 & 8 & -1.9536 E+00 \\ 8 & 3 & 4 & 2.7323 E-01 \\ 8 & 3 & 5 & 6.4308 E-02 \\ 8 & 3 & 6 & -1.3778 E-01 \\ 8 & 3 & 7 & -4.0464 E-01 \\ 8 & 3 & 8 & -9.2315 E-01 \\ 8 & 4 & 5 & 1.3897 E-01 \\ 8 & 4 & 6 & 6.4308 E-02 \\ 8 & 4 & 7 & -2.1475 E-02 \\ 8 & 4 & 8 & -1.8180 E-01 \\ 8 & 5 & 6 & 2.7323 E-01 \\ 8 & 5 & 7 & 3.4825 E-01\end{array}$

$3.8727 E+00 \quad 2.4427 E+01$

$1.4635 E+00 \quad 3.5740 E+00$

$1.8624 E+00-5.6878 E+00$

$5.8102 E+00-3.5737 E+01$

$2.4199 E+01-2.8537 E+02$

$1.5456 E+00 \quad 7.2274 E+00$

4. $9114 E-01 \quad 1.2148 E+00$

$4.2070 E-01-3.9476 E-01$

$1.3003 E+00-3.9645 E+00$

$5.8102 E+00-3.5737 E+01$

$2.5669 E-01 \quad 5.9432 E-01$

$1.6543 E-01 \quad 1.0518 E-01$

4. $2070 E-01-3.9476 E-01$

$1.8624 E+00-5.6878 E+00$

$2.5669 E-01 \quad 5.9432 E-01$

$4.9114 E-01 \quad 1.2148 E+00$

$1.4635 E+00 \quad 3.5740 E+00$

$1.5456 E+00 \quad 7.2274 E+00$

$3.8727 E+00 \quad 2.4427 E+01$

$1.3445 E+01 \quad 1.4940 E+02$

$1.6682 E+01$

$5.2905 E+00$

1. $9008 \mathrm{E}+00$

1. $2493 E+00$

$2.6302 E+00$

$7.8501 E+00$

$2.9649 E+01$

$2.1753 E+00$

7. $2731 E-01$

3. $5669 E-01$

$6.3053 E-01$

$1.9702 E+00$

$7.8501 E+00$

3.8060E-01

1. $5324 E-01$

2.0197E-01

6. $3053 E-01$

$2.6302 E+00$

$1.2594 E-01$

1. $5324 E-01$

$3.5669 E-01$

1. $2493 E+00$

3.8060E- 01

$7.2731 E-01$
$1.9148 E+02$

3. $5619 E+01$

7. $5526 E+00$

$-6.1836 E-01$

$-1.0613 E+01$

$-5 \cdot 1588 E+01$

$-3.6330 E+02$

$1.0722 E+01$

2. $2180 E+00$

$2.3637 E-01$

$-1.1958 E+00$

$-6.7333 E+00$

$-5.1588 E+01$

$9.9559 E-01$

1. $8687 E-01$

$-1.3990 E-01$

$-1.1958 E+00$

$-1.0613 E+01$ 1. $9511 E-01$

1. $8687 E-01$

2. 3637E-01

$-6.1836 E-01$

$9.9559 E-01$

$2.2180 E+00$
$2.4999 E+02$

3. $7153 E+01$

$3.6688 E+03$

4. $5311 E+01$

$3.4628 E+02$

5. $2382 E+03$

$5.5638 E+01$

$6.5164 E+00$

$2.7276 E+00$

1. $9986 E+01$

$3.4628 E+02$

$2.4790 E+0 O$

$6.6606 E-01$

2. $7276 E+00$

$4.5311 E+01$

$2.4790 E+00$

$6.5164 E+00$

$3.7152 E+01$

$5.5638 E+01$

$2.4999 E+02$

$2.6725 E+03$

$3.4867 E+03$

3. $7421 E+02$

$5.8647 \mathrm{E}+01$

$2.0125 E+01$

$7.4376 E+01$

$5.1722 E+02$

6. $8118 E+03$

$8.4957 E+01$

$1.1562 E+01$

$2.2564 E+00$

$4.6105 E+00$

$3.5361 E+01$

5. $1722 E+02$

4.3791E+00

$6.8480 E-01$

$6.0984 E-01$

$4.6105 E+00$

$7.4376 E+01$

$5.4364 E-01$

6. $8480 E-01$

$2.2564 E+00$

$2.0125 E+01$

4. $3791 E+00$

$1.1562 E+01$
2. $8577 E+02$

$-3.5833 E+02$

$-4.8420 E+03$

$-1.4050 E+05$

$6.3704 E+02$

4. $5085 E+01$

$-5.3675 E+00$

$-1.4152 E+02$

$-4.8420 E+03$

1. $5490 E+01$

$1.8225 E+00$

$-5.3675 E+00$

$-3.5833 E+02$

1. $5490 E+01$

4. $5085 E+01$

$2.8577 E+02$

6. $3704 E+02$

$3.6688 E+03$

$7.0861 E+04$

$9.3377 E+04$

$5.6214 E+03$

$5.7877 E+02$

$-1.1986 E+01$

$-7.0250 E+02$

$-7.4138 E+03$

$-1.8486 E+05$

9. $9065 E+02$

$8.5291 E+01$

$7.0029 E+00$

$-2.0689 E+01$

$-2.6235 E+02$

$-7.4138 E+03$

$2.8613 E+01$

$2.7434 E+00$

$-9.4102 E-01$

$-2.0689 E+01$

$-7.0250 E+02$

$2.2339 E+00$

$2.7434 E+00$

7. $0029 E+00$

$-1.1986 E+01$

2. $8613 E+01$

8. $5291 E+01$ 
TABLE 13. (CONTINUED)

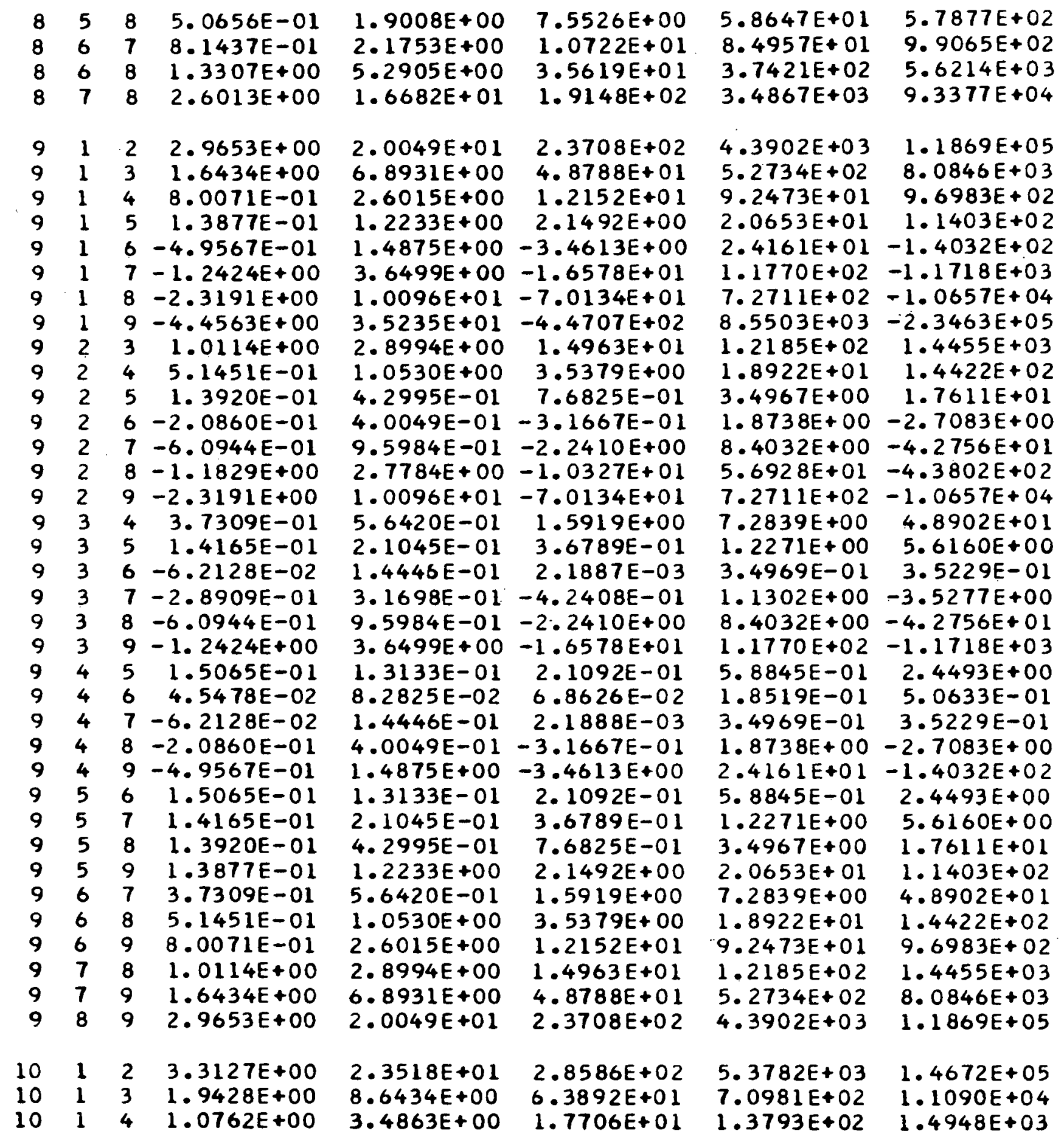


TABLE 13. (CONT INUEO)

$\begin{array}{rrrr}10 & 1 & 5 & 4.1853 E-01 \\ 10 & 1 & 6 & -1.6594 E-01 \\ 10 & 1 & 7 & -7.8129 E-01 \\ 10 & 1 & 8 & -1.5449 E+00 \\ 10 & 1 & 9 & -2.6672 E+00 \\ 10 & 1 & 10 & -4.9036 E+00 \\ 10 & 2 & 3 & 1.2088 E+00 \\ 10 & 2 & 4 & 6.8190 E-01 \\ 10 & 2 & 5 & 2.9408 E-01 \\ 10 & 2 & 6 & -4.1122 E-02 \\ 10 & 2 & 7 & -3.8656 E-01 \\ 10 & 2 & 8 & -8.1045 E-01 \\ 10 & 2 & 9 & -1.4311 E+00 \\ 10 & 2 & 10 & -2.6672 E+00 \\ 10 & 3 & 4 & 4.8543 E-01 \\ 10 & 3 & 5 & 2.3278 E-01 \\ 10 & 3 & 6 & 2.2482 E-02 \\ 10 & 3 & 7 & -1.8766 E-01 \\ 10 & 3 & 8 & -4.4124 E-01 \\ 10 & 3 & 9 & -8.1045 E-01 \\ 10 & 3 & 10 & -1.5449 E+00 \\ 10 & 4 & 5 & 1.9516 E-01 \\ 10 & 4 & 6 & 6.9200 E-02 \\ 10 & 4 & 7 & -4.9409 E-02 \\ 10 & 4 & 8 & -1.8766 E-01 \\ 10 & 4 & 9 & -3.8656 E-01 \\ 10 & 4 & 10 & -7.8129 E-01 \\ 10 & 5 & 6 & 1.1538 E-01 \\ 10 & 5 & 7 & 6.9200 E-02 \\ 10 & 5 & 8 & 2.2482 E-02 \\ 10 & 5 & 9 & -4.1122 E-02 \\ 10 & 5 & 10 & -1.6594 E-01 \\ 10 & 6 & 7 & 1.9516 E-01 \\ 10 & 6 & 8 & 2.3278 E-01 \\ 10 & 6 & 9 & 2.9408 E-01 \\ 10 & 6 & 10 & 4.1853 E-01 \\ 10 & 7 & 8 & 4.8543 E-01 \\ 10 & 7 & 9 & 6.8190 E-01 \\ 10 & 7 & 10 & 1.0762 E+00 \\ 10 & 8 & 9 & 1.2088 E+00 \\ 10 & 8 & 10 & 1.9428 E+00 \\ 10 & 9 & 10 & 3.3127 E+00\end{array}$

$1.5021 E+00$

$1.0937 E+00$

1. $9890 E+00$

4. $8574 E+00$

1. $2505 E+01$

$4.0918 E+01$

$3.7069 E+00$

$1.4544 E+00$

$5.9085 E-01$

$3.4779 E-01$

$5.5514 E-01$

1. $3876 E+00$

3. $7056 E+00$

$1.2505 E+01$

7. $9569 E-01$

$3.1096 E-01$

$1.5093 E-01$

1.9661E-01

4. $9754 E-01$

1. $3876 E+00$

4. $8574 E+00$

$1.8562 E-01$

8. 2608E-02

8. $3693 E-02$

1. $9661 E-01$

$5.5514 E-01$

$1.9890 E+00$

$7.5654 E-02$

$8.2608 E-02$

1. 5093E- 01

3.4779E-O1

1. $0937 E+00$

1.8562E-01

3. $1096 E-01$

5. $9085 E-01$

$1.5021 E+00$

7. $9569 E-01$

$1.4544 E+00$

3. $4863 E+00$

3. $7069 E+00$

$8.6434 E+00$

2. $3518 E+01$
$4.6283 E+00$ $-4.4953 E-01$

$-6.3906 E+00$

$-2.3829 E+01$

$-9.1271 E+01$

$-5.3601 E+02$

$1.9949 E+01$

$5.2195 E+00$

$1.3972 \mathrm{E}+00$

1. $4877 E-01$

$-8.6209 E-01$

$-3.6347 E+00$

$-1.4789 E+01$

$-9.1271 E+01$

$2.3809 E+00$

6.3382E-01

$1.2517 \mathrm{E}-01$

$-1.5673 E-01$

$-8.2512 E-01$

$-3.6347 E+00$

$-2.3829 E+01$

$3.3327 E-01$

8.6499E-02

$-2.8670 E-03$

$-1.5673 E-01$

$-8.6209 E-01$

$-6.3906 E+00$

8.9094E-02

$8.6499 E-02$

1. $2517 E-01$

$1.4877 \mathrm{E}-01$

$-4.4953 E-01$

3. $3327 E-01$

$6.3382 E-01$

$1.3972 E+00$

$4.6283 E+00$

2. $3809 E+00$

$5.2195 E+00$

1.7706E+01

$1.9949 E+01$

$6.3892 E+01$

$2.8586 E+02$
3. $0444 E+01$

2. $3219 E+02$

$1.3176 E+01-6.0467 E+00$

$3.7209 E+01-2.7382 E+02$

$1.7519 E+02-1.8033 E+03$

$9.7648 E+02-1.4612 E+04$

$1.0444 E+04-2.8964 E+05$

$1.6673 E+02 \quad 2.0103 E+03$

$2.8874 E+01 \quad 2.2606 E+02$

$5.8435 E+00 \quad 3.2566 E+01$

$1.6068 E+00 \quad 3.5023 E+00$

$2.8611 E+00-9.9085 E+00$

$1.4220 E+01-7.6477 E+01$

$8.5400 E+01-6.7905 E+02$

$9.7648 E+02-1.4612 E+04$

$1.1286 E+0.1 \quad 7.7601 E+01$

$2.1461 E+00 \quad 1.0308 E+01$

$4.6495 E-01 \quad 1.3816 E+00$

4.3341E-01 -7.2931E-01

$2.1753 E+00-7.7254 E+00$

$1.4220 E+01-7.6477 E+01$

$1.7519 E+02-1.8033 E+03$

$9.9141 E-01$

$2.0342 E-01$

$1.1227 E-01$

4.3341E-OI

$2.8611 E+00$

3. $7209 E+01$

1. $8100 E-01$

$2.0342 E-01$

4. $6495 \mathrm{E}-01$

$1.6068 E+00$

$1.3176 E+01$

9.9141E-01

$2.1461 E+00$

5. $8435 E+00$

$3.0444 E+01$

$1.1286 E+01$

$2.8874 E+01$

$1.3793 E+02$

$1.6673 E+02$

$7.0981 E+02$

$5.3782 \mathrm{E}+03$
4. $3401 E+00$

$6.1750 E-01$

$6.1272 E-02$

$-7.2931 E-01$

$-9.9085 E+00$

$-2.7382 E+02$

$5.4232 E-01$

6.1750E-O1

$1.3816 \mathrm{E}+00$

3. $5023 E+00$

$-6.0467 E+00$

4. $3401 E+00$

$1.0308 E+01$

3. $2566 E+01$

2. $3219 E+02$

$7.7601 E+01$

$2.2606 E+02$

$1.4948 E+03$

$2.0103 E+03$

$1.1090 E+04$

$1.4672 E+05$ 
half normal. This procedure is much more efficient than the usual direct integration technique used, for example, by Sarhan (36) to generate the moments of the double exponential. Sarhan gives the equation

$$
E\left(X_{(r)}^{m}\right)=\int_{-\infty}^{0} x(\stackrel{m}{r})^{f_{1}}(x(r)) d x+\int_{0}^{\infty} x(r) m_{f_{2}}(x(r)) d x
$$

where

$$
f_{1}(x(r))=\frac{n !}{(r-1) !(n-r) !}\left(\frac{1}{2} e^{x}(r)\right)\left(1-\frac{1}{2} e^{x}(r)\right)^{n-r}
$$

and

$$
f_{2}(x(r))=\frac{n !}{(r-1) !(n-r) !}\left(1-\frac{1}{2} e^{-x}(r)\right)\left(\frac{1}{2} e^{x}(r)\right)^{n-r+1}
$$

The functions $f_{1}$ and $f_{2}$ above must be expanded using the binomial theorem and then integrated by parts. The procedure as followed by Sarhan becomes intolerable as sample size and moments increase. 
CHAPTER VII. SUMMARY

Let $X_{(1)}$ be the ordered random variables for a sample of $n$ observations selected from an exponential with parameter b. Then 1t is known that

$$
z_{k}=(n-k+1)\left(x_{(k)}-x_{(k-1)}\right) \quad k=1,2, \ldots, n
$$

where $X_{(0)}=0$ are identically and independently distri-. buted as exponential with parameter b. Furthermore we showed that $\mathrm{X}_{(1)}$ is independent of $\mathrm{z}_{\mathrm{g}}$ if $\mathrm{i}<\mathrm{j}$.

If $Y$ is a Weibull random variable with density

$$
\begin{gathered}
f(y, a, b)=\frac{1}{a b}(y)^{\frac{1}{a}-1} \exp \left(\left(y^{1 / a} / b\right)\right) \quad j>0, a>0, \\
b>0
\end{gathered}
$$

the mean and variance are given by

$$
\begin{aligned}
& E(y)=b^{2} r(a+1) \\
& V(y)=b^{2 a}\left(r(2 a+1)-(r(a+1))^{2}\right) .
\end{aligned}
$$

The transformation $X=Y^{1 / a}$ is one to one, order preserving and the random variable $\mathrm{X}$ is distributed as an exponential with parameter b. Now from 7.1

$$
x_{(k)}=x_{(k-1)}+z_{k} /(n-k+1)
$$

and

$$
E(Y(k))=E\left(X_{(k)}^{a}\right)=E\left(\left(X(k-1)+Z_{k} /(n-k+1)\right)^{2}\right) . \quad(7 \cdot 4)
$$


If a is an integer we use the binomial theorem to obtain

$$
E\left(Y_{(k)}\right)=a d b^{a} \cdot \sum_{i=0}^{a} E\left(X_{(k-1)}^{i}\right)\left(\frac{1}{n-k+1}\right)^{a-i} / 1 d
$$

We may rewrite Equation 7.3 as

$$
x_{(k)}=x_{(k-j)}+w_{k, j}
$$

where $W_{k, j}$, defined in Equation 2.20, is a wolghted sum of 2 's. Hence by the binomial theorem

$$
\begin{aligned}
E\left(Y_{(k-j)} Y_{(k)}\right) & =E\left(\left(X_{(k-j)}\left(X_{(k-j)}+W_{k, j}\right)\right)^{a}\right) \\
& =E\left(\left(X_{(k-j)}^{2}+X_{(k-j)} W_{k, j}\right)^{a}\right) \\
& =b^{2 a} \sum_{i=0}^{a}\left(\begin{array}{l}
a \\
a
\end{array}\right) E\left(X_{(k-j)}^{a+j}\right) E\left(w_{k, j}^{a-j}\right) .
\end{aligned}
$$

Both of the expected values contained in the expression on the right hand side of Equation 7.7 are known. Hence Equations 7.5 and 7.7 may be used to compute the variances and covariances of the Weibull with integer shape parameter.

The asymptotic expectations and asymptotic covariances of Weibull order otatisties were developed by deriving the asymptotic moment generating functions. Asymptotically the largest exponential order statistic has an extreme value distribution. Hence the $m g f$ of the largest order statistic of an exponential distribution is

$$
G_{X_{(n)}}(t)=\exp (b t \log n) \Gamma(1-b t)
$$


Using the independence of $z_{k}$ and $x_{1}$ we have the mgf of the lower order statistics,

$$
G_{X_{(n-k)}}(t)=\exp (b t \log n) \Gamma(1-b t) \prod_{j=1}^{k}\left(1-\frac{b t}{j}\right) .
$$

The asymptotic expectations are

$$
\begin{gathered}
E^{*}(Y(n))=E^{*}\left(X_{(n)}^{a}\right)=b^{a} \sum_{i=0}^{a}\left({ }_{i}^{a}\right)(1 \circ g n)^{a-1} I(1) \\
E^{*}\left(Y_{(n-k)}\right)=E^{*}\left(X_{(n-k)}^{a}\right)=b^{a} \sum_{i=0}^{a}\left(a_{i}^{a}\right) h(1, k, 0) E^{*}\left(X_{(n)}^{a-1}\right) \\
k=1,2, \ldots
\end{gathered}
$$

where $I(1)=\left|\int_{0}^{\infty} e^{-z}(\log z)^{1} d z\right|$ and $h(1, k, 0)=\frac{d_{d}^{j}}{d t} \prod_{j=1}^{k}(1-t / g)$ evaluated at $t=0$. Using Equation 7.5 the as ymptotic expectations of the crossproducts are given by

$$
\begin{aligned}
E^{*}\left(Y(k-j) Y_{(k)}\right) & =E^{*}\left(X_{(k-j)}^{a} X_{(k)}^{a}\right) \\
& =\sum_{1=0}^{a}\left(\begin{array}{l}
a \\
1
\end{array}\right) E^{*}\left(X_{(k-j)}^{a+1}\right) E\left(w_{k, j}^{a-i}\right)
\end{aligned}
$$

and asymptotic variances and covariances may be calculated in the usual manner.

The expected values and covariances of the order statistics of the exponential are well known.

$$
\begin{aligned}
& E\left(X_{(k)}\right)=b \sum_{j=1}^{k} 1 /(n-j+1) \\
& V\left(X_{(k)}\right)=b^{2} \sum_{j=1}^{k} 1 /(n-j+1)^{2} \\
& C\left(X_{(j)} X_{(k)}\right)=V\left(X_{(j)}\right) \text { if } j<k .
\end{aligned}
$$


The asymptotic approximations are given by

$$
\begin{aligned}
& E^{*}(X(n-k))=10 g n+I(1)-\sum_{j=1}^{k} I / j \\
& V^{*}(X(n-k))=\frac{\pi^{2}}{6}-\sum_{j=1}^{k} I / j^{2} \quad k=1,2, \ldots .
\end{aligned}
$$

Letting $a=1$ in Equation 7.10 we have $E^{*}\left(X_{(n)}\right)=b(\log n+$ $I(1))$. The error incurred by using the asymptotic formula ranges from about four percent at $n=5$ to 0.1 percent at $n=100$. Also $V^{*}\left(X_{(n)}\right)=\pi^{2} / 6$ which is independent of sample size and differs from $V\left(X_{(n)}\right)$ by about two percent at $n=25$.

The class of estimators of the mean

$$
\widehat{w}=\left(\sum_{i=0}^{n-r} X_{(i)}+r X_{(n-r)}\right) / D
$$

is introduced. In terms of the random variabies $z_{i}$, Equation 7.15 may be written

$$
\hat{v}=\sum_{i=0}^{n-r} z_{i} / D \text {. }
$$

Three members of this class are studied as estimators for the mean of the Weibull distribution. These are i) the winsorized mean where $D=n$, il) the estimate due to Epstein where $D=n-r$, and $11 i$ ) the member of the class derived to minimize the mean square error of $\hat{w}$ for the exponential distribution where $D=n-r+1$. The MSE of these estimates is compared to the MSZ of $Y$. There is a large gain in efficiency with respect to the mean in using $\hat{w}$ instead of Pas the shape parameter incruasus. An argument is 
presented to show that in large samples from a Welbull with shape parameter greater than one $\operatorname{MSE}\left(\hat{w}_{1}\right)<\operatorname{MSE}(Y)$ where $\hat{W}_{1}$ is the once consored Winsorized mean.

The maximum likelihood estimator of the mean of the Woibull is given by

$$
\hat{m}=\hat{c} r(\hat{a}+1)
$$

where $c$ and $a$ are the scale and shape parameters respectively. Employing a Tajlor series expansion the asymptotic variance of $\hat{m}$ is expressed as a function of the asymptotic variances and covariances of $\hat{c}$ and $\hat{a}$. As the shape parameter increases above one large galns in efficlency are mede by using the MLE Instead of $\bar{Y}$ as an ostimator of the mean.

Three tests for the shape parameter of the Welbull are investigated in a Monte Carlo study. The first test statistic may be written

$$
T=\frac{n}{\Sigma} D_{k} Z_{k}^{\prime} \sum^{n} Z_{k}
$$

where the $D_{k}$ are constants and

$$
\left.z_{k}=(n-k+1)(Y / k)-Y_{(k-1)}^{1 / a_{0}}\right)
$$

The $Y_{(k)}$ are the Wolbull order statistics and $a_{0}$ is the hypothesized value of the shape parameter. If the null hypothesis is true $E(T)=0$. If the true value of the ohape parameter differs from a thon $E(T)$ diffora from 
zero. Hence $T$ is intultively appoaling for testing $\mathrm{H}_{0}: a=a_{0}$ versis $\mathrm{H}_{\mathrm{A}}: a \neq a_{0}$. The distribution of $\mathrm{T}$ is known.

The second test is derived from the extreme value distribution. We know that if $Y$ is a Welbull variate then log $Y$ has the extreme value distribution. The expected values and covariances of the order statistics of the extreme value distribution are known. The parameters of this distribution are of the location and scale trpe so that least squares may be used to obtain linear estimators of the parameters. A test statistic analogous to student's $t$ is

$$
W=\frac{\hat{B}-a_{0}}{\sqrt{V(\hat{B})}}
$$

where $\hat{B}$ is the least squares estimate of a. Little is known of the distribution of $W$.

, The third test investigated is the likelihood ratio test for $\mathrm{H}_{0}: a=a_{0}$ versus $\mathrm{H}_{\mathrm{A}}: a=a_{1}$. The test statistic is

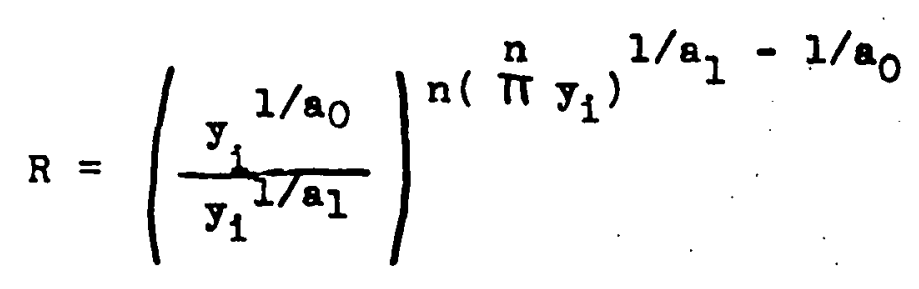

whoro $H_{0}$ is accopted if $R>k$. The distribution of $R$ is not known for this test so that Monte Carlo methods were used to establish critical values. 
When the three tests were compared using Monte Carlo methods the $T$ and $W$ statistics were found to have approximately the same power with $W$ generally more powerful. The likelihood ratio test was the most powerful when the underlying distribution used for the alternative was Weibull with $a=a_{1}$. The likelihood ratio test would be expected to have less power if the alternative were not that used in constructing the test.

A combinatorial form is developed expressing the expected values and expected crossproducts of the order statistics of a symmetric distribution as a function of the expected values and expected crossproducts of the order statistics of the related half distribution. If $z$ is the symmetric random variable then $g(z)=\frac{I}{2} f(z)$ if $z>0$ and $g(z)=\frac{1}{2} f(-z)$ if $z<0$ where $f(y)$ is the density of the half distribution. It is argued that the conditional density of the $j$ largest observations of a sample of size $n$ from the symmetric variate given that $J=f$ of. them are greater than zero is precisely that of a sample of size $f$ from the density of the positive half distribution. The number of observations greater than zero is a binomial random variable with parameter $p=\frac{1}{2}$. The expected values of the order statistics of the symmetric random variables are then givon by the summation over the random variable J. The form for the crossproducts is developed in a similar manner. In particular the expected values 
$119-121$

and expected crossproducts of the double Weibull distribution are tabulated. 


\section{BIBLIOGRA PHY}

1. Bartholomew, D. J. The sampling distribution of an est1mate arising in life testing. Technometrics 5: 361374. 1963.

2. Blom, G. Statistical estimates and transformed beta variables. New York, N.Y., John Wlley and Sons, Inc. 1958.

3. Cochran, W. G. Sampling techniques. New York, N.Y., John Wiley and Sons, Inc. 1963.

4. David, F. N. and Johnson, N. L. Statistical treatment of censored data. B1ometrica 41: 228-240. 1954.

5. Dixon, W. J. Simplified estimation from censored normal samples. Annals of Math. Stat. 31: 385-391. 1960.

6. Dixon, W. J. and Tukey, J. W. Approximate behavior of the distribution of Winsorized t. Technometrics 10: 83-98. 1968.

7. Dodd, E. I. The greatest and the least varlate under general laws of error. Am. Math. Soc. Transactions 25: 525-539. 1923 .

8. Downton, F. Inear estimates of the parameters in the extreme value distribution. Technometrics 8: 3-17. 1966.

9. Dubey, S. D. On some permissible estimators of the location parameter of the Welbull and certain other distrihutions. Technometrics 9: 293-307. 1967.

10. Epstein, B. Estimation from life test data. Technometrics $2: 447-454.1960$.

11. Epste1n, B. Estimation of the parameters of the two parameter exponential distribution from censored samples. Technometrics $2: 435-446.1960$.

12. Epstein, B. Tests for the validity of the assumption that the underlying distribution of life is exponential. Technometrics 2: 83-101, 167-183. 1960.

13. Epste1n, B. Truncated Iife tests in the exponential case. Ann. Math. Stat. 25: 554-564. 1954. 
14. Epstein, B. and Sobe1, M. Life testing. J. Am. Stat. Assn. 48: 486-502. 1953.

15. Epstein, B. and Sobel, M. Some theorems relevant to life testing from an exponential distribution. Ann. Math. Stat. 25: 373-381. 1954.

16. Gumbel, E. J. Statistics of extremes. New York, N.Y., Columbia University Press. 1958.

17. Fisher, R. A. Tippett, L. Limlting forms of the frequency distribution of the largest or smallest member of a sample. Cambridge Philosophical Soc. Proceedings 24: 180-190. 1927.

18. Fuller, W. A. Comments on estimation for the Weibuli. Unpublished Report. Ames, Iowa, Dept. of Stat., Iowa State Univ. ca. 1967.

19. Halperin, M. Maximum likelihood estimation in truncated samples. Ann. Math. Stat. 23: 226-238. 1952.

20. Harter, H. L. and Moore, A. H. Conditional maximum likelihood estimators from singly censored samples of the scale parameters of the type II extreme value distributions. Technometrics 10: 349-359. 1968.

21. Harter, H. L. and Moore, A. H. Maximum likelihood est1mation from doubly censored samples of parameters of the first asymptotic distribution of extreme values. J. Am. Stat. Assn. 63: 889-894. 1968.

22. Harter, H. I. and Moore, A. H. Maximum likelihood est1mation of the parameters of gamma and Welbull populations from complete and from censored samples. Technometrics 7: 639-643. 1965.

23. Harter, H. L. and Moore, A. H. Point and interval estimators based on $m$ order statistics for the scale parameter of a Welbull population with a known shape parameter. Technometrics 7: 405-422. 1965.

24. Jackson, O. A. Y. An analysis of departures from the exponential distribution. J. Royal Stat. Soc, Series B\#3: 540-549. 1967.

25. Johns, M. V. and Lieberman, G. J. An exact asymptotically efficient confidence bound for reliability in the case of the Welbull distribution. Technometrics 8: 135-175. 1966. 
26. Lieblein, J. and Zelen, M. Statistical investigation of the fatigue life of deep-groove ball bearings. Nat. Bur. Stand. J. Res. 5: 273-316. 1956.

27. Lloyd, E. H. Least squares estimation of location and scale parameters using order statistics. Blometrics 39: 88-95. 1952 .

28. McElhone, D. H. and Larsen, N. C. Expected values, varlance and covariances of order statistics drawn from a welbull density with integer shape parameter. U.S. Atomic Energy Commission Report IS-2156 (Iowa State Un1v. Institute of Atomic Research., Ames). 1969.

29. McElhone, D. H. and Larsen, N. C. Expected values and variances of order statistics drawn from a double Weibull density with integer shape parameter. U.S. Atomic Energy Commission Report IS-2182 (Iowa State Univ. Institute of Atomic Research., Ames). 1969.

30. Mann, N. R. Point and interval estimation for the two parameter Weibull and extreme value distribution. Technometrics 10: 231-256. 1968.

31. Menon, M. B. Estimation of the shape and scale parameters of the Welbull distribution. Technometrics 5: 175-182. 1963.

32. Renyi, A. On the theory of order statistics. Acta Math. Acad. Sclence Hungar. 4: 191-227. 1953.

33. Rosenzwe1g, M. S. Ordered estimators for skewed populations. Unpublished Ph.D. thesis. Ames, Iowa, Library, Iowa State University. 1968.

34. Rosenzwe1g, M. S. and Fuller, W. A. An estimation procedure for highly skewed distributions. Unpublished paper. Ames, Iowa, Iowa State Univ., Dept. of Stat. ca. 1969.

35. Saleh, A. K. Determination of the exact optimum order statistics for estimating the parameters of the exponential distribution from censored samples. Technometrics 9: $279-285$. $196 \%$

36: Sarhan, A. E. Estimation of the mean and standard deviation by order statistics. Ann. Math. Stat. 25: 317-328. 1954. 
37. Sarhan, A. E. and Greenberg, B. G., eds. Contributions to order statistics. New York, N.Y., John Wiley and Sons, Inc. 1962.

38. Sarhan, A. E. and Greenberg, B. G. Est1mation of location and scale parameters by order statistics from singly and doubly censored samples. Ann. Math. Stat. 27: 427-451. 1956.

39. Searles, D. T. An estimator for a population mean which reduces the effect of large true observations. J. Am. Stat. Assn. 61: 1200-1204. 1966.

40. Splitstone, D. E. Estimation of the Weibull shape and scale parameters. Unpublished M.S. thesis. Ames, Iowa, Library, Iowa State University. 1967.

41. Thoman, D. R., Bain, I. J. and Antle, C. E. Inferences on the parameters of the Welbull distribution. Technometrics 11: 445-460. 1969.

42. Tukey, J. W. The future of data analysis. Ann. Math. Stat. 33: 1-67. 1962.

43. Tukey, J. W. and McLaughlin, D. H. Less vulnerable confidence and significance procedures for location based on a single sample. Sankya the Indian J. of Stat. Series A, 25, Part 3: 331-352. 1963.

44. U.S. Dept. of Commerce. Handbook of mathematical functions. National Bureau of Standards Applied Mathematics Series 55. 1964.

45. Welbull, W. A statistical distribution of wide applicab1lity. J. Appl. Mech. 18: 293-297. 1951.

46. Weibull, W. A statistical theory of the strength of material. Ing. Vetenskaps Akad. Handl. 153: 17-42. 1939.

47. White, J. S. Least squares unblased censored Iinear estimation for the log Weibull distribution. Industrial Mathematics 14: 21-59. 1964.

48. Wilks, S. S. Mathemat1cal statistics. New York, N.Y., John Wiley and Sons, Inc. 1962.

49. Wliks, S. S. Order statist1cs. Bull. Ann. Math. Soc. $54: 6-50.1948$. 


\section{APPENDIX}

Presented here is the derivation of the limiting covariance matrix of the maximum likelihood estimates of parameters $c$ and $a$ of the Welbull distribution. The maximum likelibood estimates $\hat{c}$ and $\hat{a}$ for the parameters of the density $f(y ; a, c)$ based on a sample of size $n$ are approximately distributed, for large $n$, as a multivariate normal with means $c$ and a and covariance matrix given by

$$
\$=-\frac{1}{n}\left(\begin{array}{cc}
E\left(\frac{\partial^{2}}{\partial c^{2}} \log f(y ; a, c)\right) & E\left(\frac{\partial^{2}}{\partial c \partial a} \log f(y ; a, c)\right) \\
0 & E\left(\frac{\partial^{2}}{\partial a^{2}} \log f(y ; a, c)\right)
\end{array}\right)^{-1}
$$

The density of the Welbull with scale parameter $c$ and shape parameter a is

$$
f(y ; a, c)=\frac{1}{a c}\left(\frac{y}{c}\right)^{\frac{1}{a}-1} \exp \left(-(y / c)^{\frac{1}{a}}\right) \quad y>0
$$

Hence

$$
\log f(y ; a, c)=-\log a-\log c+\left(\frac{1}{a}-1\right) \log (y / c)-(y / c)^{\frac{1}{a}} .
$$

Taking the partial derivatives we have

$$
\begin{aligned}
& \frac{\partial^{2}}{\partial c^{2}} \log f(y ; a, c)=\frac{1}{a c^{2}}-\frac{1}{a c^{2}}(y / c)^{1 / a}-\frac{1}{a^{2} c^{2}(y / c)^{1 / a}} \\
& \frac{\partial^{2}}{\partial c \partial a} \log f(y ; a, c)=\frac{1}{a^{2} c}-\frac{1}{a^{2} c}(y / c)^{1 / a}-\frac{1}{a^{3} c}(y / c)^{1 / a} \log (y / c) \\
& \frac{\partial^{2}}{\partial a^{2}} \log f(y ; a, c)=\frac{1}{a^{2}}+\frac{2}{a^{2}} \log (y / c)-\frac{2}{a^{3}}(y / c)^{1 / a} \log (y / c)
\end{aligned}
$$




$$
-\frac{1}{a^{4}}(y / c)^{1 / a}(\log (y / c))^{2} \text {. }
$$

Furthermore using the transformation $z=(\mathrm{g} / \mathrm{c})^{1 / a}$, wo find the expected values

$$
\begin{aligned}
& E\left((y / c)^{1 / a}\right)=\Gamma(2) \\
& E(\log (y / c))=\Gamma^{(1)}(1) a \\
& E\left((y / c)^{1 / a} \log (y / c)\right)=\Gamma^{(1)}(2)_{a} \\
& E\left((y / c)^{1 / a}(\log (y / c))^{2}\right)=\Gamma^{(2)}(2)_{a}^{2} .
\end{aligned}
$$

From $(44)$ wo know that $r(2)=1, \Gamma^{(1)}(2)=1-.5772$, $r(2)(2)=\left(\frac{\pi^{2}}{6}-1\right)+(1-.5772)^{2}$, and $\Gamma^{(1)}(1)=-.5772$.

\section{Hence}

$$
\begin{aligned}
\frac{1}{4} & =\frac{1}{n}\left(\begin{array}{ll}
\frac{1}{(\mathrm{ac})^{2}} & \frac{.4228}{c a^{2}} \\
\mathrm{sym} . & \left.\frac{1}{a^{2}\left(\frac{\pi^{2}}{6}+(.4228)^{2}\right.}\right)
\end{array}\right)^{-1} \\
& =\frac{1}{n}\left(\begin{array}{ll}
1.1087(\mathrm{ac})^{2} & -.2570 c a^{2} \\
-.2570 c a^{2} & .6079 a^{2}
\end{array}\right)
\end{aligned}
$$

which is exactiy Equation 5.8 . 